\title{
Thiazolium-Catalyzed Additions of Acylsilanes: A General Strategy for Acyl Anion Addition Reactions
}

\author{
Anita E. Mattson, Ashwin R. Bharadwaj, Andrea M. Zuhl and Karl A. Scheidt* \\ Department of Chemistry, Northwestern University, 2145 Sheridan Road, Evanston, Illinois 60208
}

\section{Supplemental Section Table of Contents}

1. General methods

S2-S3

2. Procedure for Thiazolium-Catalyzed Acylsilane Additions

to $\alpha, \beta$-Unsaturated Systems and Characterization Data for 1,4-Diketones (4-34) S3-S8

3. Procedure for Thiazolium-Catalyzed Furan Synthesis and S8-S9

Characterization Data for Furan Products (25-30)

4. Procedure for Thiazolium-Catalyzed Pyrrole Synthesis and

S9-S13

Characterization Data for Pyrrole Products (31-49)

5. Microwave Procedure for Preparation of $\mathbf{3 6}$

S13

6. Procedure for Thiazolium-Catalyzed $\alpha$-Aminoketone Synthesis

S14-S16 and Characterization Data for $\alpha$-Aminoketones (51-60)

7. Synthesis of Proposed Intermediate II

S17-S18

8. References

S18-S19

9. ${ }^{1} \mathrm{H}$ and ${ }^{13} \mathrm{C}$ Spectra

S20-S90

1,2,4-Triphenylbutane-1,4-dione (4)

S20-S21

4-(4-chlorophenyl)-1-2-diphenylbutane-1-4-dione (5)

S22-S23

4-(4-methoxyphenyl)-1,2-diphenylbutane-1-4-dione (6)

$\mathrm{S} 24-\mathrm{S} 25$

2-(Naphthalene-4-yl)-1,4-diphenylbutane-1,4-dione (7)

S26-S27

2-(4-Bromophenyl)-1,4-diphenylbutane-1,4-dione (8)

S28-S29

2-(4-Chlorophenyl)-1,4-diphenylbutane-1,4-dione (9)

S30-S31

2-(2-Chlorophenyl)-1,4-diphenylbutane-1,4-dione (10)

S32-S33

(2-(4-Methylphenyl)-1,4-diphenylbutane-1-4-dione (11)

S34-S35

2-(3-Methoxyphenyl)-1,4-diphenylbutane-1,4-dione (12)

S36-S37

2-(4-Methoxyphenyl)-1,4-diphenylbutane-1,4-dione (13)

S38-S39

1-(4-chlorophenyl)-2-4-diphenylbutane-1,4-dione (15)

S40-S41

2,4-diphenyl-1-p-tolylbutane-1,4-dione (16)

$\mathrm{S} 42-\mathrm{S} 43$

1,3-Diphenylpentane-1,4-dione (17)

S44-S45

1-Cyclohexyl-2-4-diphenyl-1-4-dione (18)

$\mathrm{S} 46$

Diethyl 2-benzoylsuccinate (19)

S47-S48 
Dimethyl 2-benzoylsuccinate (20)

1-Phenylpentane-1,4-dione (22)

S49-S50

1-(4-chlorophenyl)-2-phenylpentane-1,4-dione (23)

S51-S52

2, 3, 5 triphenylfuran (25)

S53-S54

3-(3,4-dichlorophenyl)-2-ethyl-5-phenylfuran (27)

S55-S56

3-(4-bromophenyl)-2-ethyl-5-phenylfuran (28)

S57-S58

2-methyl-5-phenyl-4- $p$-furan (30)

S59

1,2,3,5-tetraphenyl-1- $H$-pyrrole (31)

S60

2-(4-chlorophenyl)-1,3,5-triphenyl-1- $H$-pyrrole (33)

S61

2-cyclohexyl-1, 3, 5-triphenyl-1 $H$-pyrrole (34)

S62

2-methyl-1, 3, 5-triphenyl-1 $H$-pyrrole (35)

S62

1,2,5-triphenyl-3- $p$-tolyl-1- $H$-pyrrole (36)

S63

3-(-4-chlorophenyl)-1,2,5-triphenyl-1 $H$-pyrrole (37)

S64

2-(4-chlorophenyl)-3-(4-methoxyphenyl)-1,5-diphenyl-1 $H$-pyrrole (38)

1-(4-Chlorophenyl)-2,3,5-triphenyl-1- $H$-pyrrole (39)

S65

1-(4-bromophenyl)-2,3,5-triphenyl-1- $H$-pyrrole (40)

S65

S66

4-(2,3,5-triphenyl-1H-pyrrol-1yl)-phenol (41)

S67

1-methyl-2,3,5-triphenyl-1- $H$-pyrrole (43)

S68

2,3,5-triphenyl-1-propyl-1- $H$-pyrrole (44)

S69

S69

1-cyclohexyl-2,3,5-triphenyl-1H-pyrrole (46)

S70

2,3,5-triphenyl-1-((S)-1-phenylethyl)-1- $H$-pyrrole (47)

S71

(R)-1-sec-butyl-2,3,5-triphenyl-1H-pyrrole (48)

S72

4-(2,3,5-triphenyl-1 $H$-pyrrol-1-yl)benzeneamine (49)

S73

2-(Diphenylphosphinamide)-1,2-diphenylethanone (51)

S74-S75

1-(4-Chlorophenyl)-2-(diphenylphosphinamide)-2-phenylethanone (52)

S76-S77

1-(Diphenylphosphinamide)-1-phenylpropan-2-one (54)

S78

5-(Benzyloxy)-1-(diphenylphosphinamide)-1-phenylpentan-2-one (55)

S79-S80

2-(4-Chlorophenyl)-2-(diphenylphosphinamide)-1-phenylethanone (56)

S81-S82

2-(Diphenylphosphinamide)-1-phenyl-2-p-tolylethanone (57)

S83-S84

2-(Diphenylphosphinamide)-2-(4-methoxyphenyl)-1-phenylethanone (58) S85-S86

2-(2-Chlorophenyl)-2-(diphenylphosphinamide)-1-phenylethanone (59)

S87-S88

2-(Diphenylphosphinamide)-1-phenyl-2-(thiophen-2-yl)ethanone (60)

S89-S90

General Methods: Tetrahydrofuran (THF), dicholormethane (DCM) and chloroform were purified by passage through a bed of activated alumina. ${ }^{1}$ Purification of reaction products was carried out by flash chromatography using silica gel 60 (230-400 mesh). Analytical thin layer chromatography was performed on $0.25 \mathrm{~mm}$ silica gel $60-\mathrm{F}$ plates. Visualization was accomplished with UV light and potassium permangenate, ceric ammonium molybdate or anisaldehyde stains followed by heating. Melting points (mp) were obtained on a capillary melting point apparatus and are uncorrected. Infrared spectra (IR) were obtained on a spectrophotometer. Infrared spectra for all products were obtained as a thin film. Nuclear 
magnetic resonances were recorded in deuterated solvents on a $500 \mathrm{MHz}$ spectrometer. Proton $\left({ }^{1} \mathbf{H}\right.$ NMR) are reported in parts per million (ppm, d) with the residual protio solvent referenced $\left(\mathrm{CDCl}_{3}, \mathrm{~d} 7.27 ; \mathrm{D}_{2} \mathrm{O}, \mathrm{d}\right.$ 4.80; DMSO, d 2.50). ${ }^{1} \mathrm{H}$ NMR splitting patterns are designated as singlet (s), doublet (d), triplet (t), quartet (q), or septet (sep). Splitting patterns that could not be interpreted or easily visualized are designated as multiplet (m) or broad (br). Coupling constants are reported in Hertz $(\mathrm{Hz})$. Proton-decoupled $\left({ }^{13} \mathbf{C} \mathbf{N M R}\right)$ spectra are reported in ppm using the solvent as an internal standard $\left(\mathrm{CDCl}_{3}, \mathrm{~d}\right.$ 77.0; DMSO, $\mathrm{d}$ 39.5). Electrospray mass spectra (ESI-MS) were obtained using a HPLC/MS/MS Mass Spectrometer. Gas Chromatography (GC) analysis data were obtained on GC system with a capillary $30 \mathrm{~m}$ column. Unless otherwise noted, all other commercially available reagents and solvents were used without further purification.

Benzoyltrimethylsilane (1a), $p$-chlorobenzoyltrimethylsilane (1b), and $p$-toluoyltrimethylsilane (1c) were prepared according to the procedure of Yamamoto and coworkers. ${ }^{2}$ Benzoyldimethylphenylsilane (1d) were prepared using the procedure developed by Fleming and Ghosh. ${ }^{3}$ Alkyl acylsilanes (1e-1f) were prepared according to the procedure of Scheidt and coworkers. ${ }^{4}$

$\alpha, \beta$-Unsaturated ketones were prepared according to the general procedure of Murphy and Wattansin. ${ }^{5}$

\section{General Procedure for Thiazolium-Catalyzed Acylsilane Additions to $\alpha, \beta$-Unsaturated Systems:}

A screw-capped test tube was charged with the thiazolium salt (30 $\mathrm{mg}, 0.119 \mathrm{mmol})$ in a nitrogen-filled drybox. The test tube was removed from the box and placed under a positive pressure of nitrogen. Benzoyltrimethylsilane $(140 \mathrm{mg}, 0.768 \mathrm{mmol})$ in THF $(0.25 \mathrm{~mL})$ was added by syringe to the test tube followed by the addition of DBU $(17 \mathrm{~mL}, 0.119 \mathrm{mmol})$. The reaction mixture was heated to $70^{\circ} \mathrm{C}$ after which the chalcone $(0.384 \mathrm{mmol})$ in THF $(0.25 \mathrm{~mL})$ was added by syringe followed by the addition of isopropanol $(120 \mathrm{~mL}, 1.56 \mathrm{mmol})$. The reaction was allowed to stir at $70^{\circ} \mathrm{C}$ for 24 hours. Upon completion by TLC $(40 \%$ ether/hexanes), the reaction was cooled to room temperature, diluted with ethyl acetate $(20 \mathrm{~mL})$ and washed with water $(20 \mathrm{~mL})$. The aqueous layer was washed with ethyl acetate $(3 \times 30 \mathrm{~mL})$ and the combined organic extracts were dried over sodium sulfate, filtered, and concentrated in vacuo. The resulting residue was purified by flash column chromatography on silica gel.

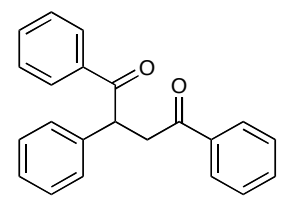

1,2,4-Triphenylbutane-1,4-dione (4): Purified with $10 \%$ ether/hexanes, yielding $93 \mathrm{mg}(77 \%)$ of $\mathbf{4}$ as a colorless oil. $\mathrm{R}_{f}=0.50$ (40/60 ether/hexanes); IR (film) $3054,2986,1681,1598 \mathrm{~cm}^{-1}$; ${ }^{1} \mathrm{H}$ NMR $\left(500 \mathrm{MHz}, \mathrm{CDCl}_{3}\right) \delta 8.06-$ 
7.98 (m, 4H); 7.58-7.54 (m, 1H); 7.49-7.39 (m, 6H); 7.37-7.32 (m, 2H); 7.26-7.22 (m, 1H); $5.34(\mathrm{dd}, \mathrm{J}=10.1,3.5 \mathrm{~Hz}, 1 \mathrm{H}) ; 4.23(\mathrm{dd}, \mathrm{J}=17.9,10.1 \mathrm{~Hz}, 1 \mathrm{H}) ; 3.32(\mathrm{dd}, \mathrm{J}=17.9,3.5 \mathrm{~Hz}, 1 \mathrm{H})$; ${ }^{13} \mathrm{C}$ NMR $\left(125 \mathrm{MHz}, \mathrm{CDCl}_{3}\right) \delta$ 199.0, 198.2, 138.8, 136.6, 133.5, 133.1, 129.4, 129.1, 128.8, 128.7, 128.4, 128.4, 127.6, 48.9, 44.2; LRMS (electrospray): Mass calculated for $\mathrm{C}_{22} \mathrm{H}_{18} \mathrm{O}_{2}$ $[\mathrm{M}+\mathrm{Na}]^{+}, 337.1$. Found 337.0. All spectral data are similar to Henrick and coworkers. ${ }^{6}$

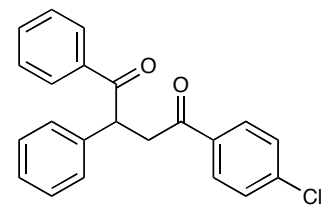

4-(4-chlorophenyl)-1-2-diphenylbutane-1-4-dione (5): Purified with 10\% ether/hexanes yielding $77 \mathrm{mg}(82 \%)$ of $\mathbf{5}$ as a clear oil. $\mathrm{R}_{f}=0.44(40 / 60$ ether/hexanes); IR (film): 3060, 2932, 1679, 1590, $1492 \mathrm{~cm}^{-1} ;{ }^{1} \mathrm{H}$ NMR (500 $\mathrm{MHz}_{\mathrm{CDCl}}$ ) $\delta$ 8.15-8.13 (m, 4H), 7.74-7.41 (m, 10H), $5.42(\mathrm{dd}, \mathrm{J}=9.8,3.3$ $\mathrm{Hz}, 1 \mathrm{H}), 4.20(\mathrm{dd}, J=18.1,4.0 \mathrm{~Hz}, 1 \mathrm{H}), 3.47(\mathrm{dd}, J=18.0,3.5 \mathrm{~Hz}, 1 \mathrm{H})$; ${ }^{13} \mathrm{C}$ NMR $\left(125 \mathrm{MHz}, \mathrm{CDCl}_{3}\right) \delta$ 199.0, 197.2, 139.9, 138.7, 136.6, 135.1, 133.2, 129.8, 129.5, 129.2, 128.8, 128.5, 127.7, 49.0, 44.1; Mass calculated for $\mathrm{C}_{22} \mathrm{H}_{17} \mathrm{ClO}_{2}[\mathrm{M}+\mathrm{Na}]^{+}, 371.1$. Found 371.0 .

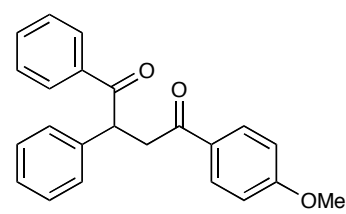

4-(4-methoxyphenyl)-1,2-diphenylbutane-1-4-dione (6): Purified with 15 ether/hexanes yielding $75 \mathrm{mg}(79 \%)$ of $\mathbf{6}$ as yellow crystals. $\mathrm{R}_{f}=0.35$ (40/60 ether/hexanes); IR (film): 3061, 2917, 1680, 1588, $1570 \mathrm{~cm}^{-1} ;{ }^{1} \mathrm{H}$ $\operatorname{NMR}\left(500 \mathrm{MHz}, \mathrm{CDCl}_{3}\right) \delta$ 8.18-8.12 (m, 4H), 7.70-7.41 (m, 8H), 6.99 (d, $J=8.7,2 \mathrm{H}), 5.44(\mathrm{dd}, J=9.9,3.67,1 \mathrm{H}), 4.33(\mathrm{dd}, J=18.1,9.9,1 \mathrm{H})$, $3.90(\mathrm{~s}, 3 \mathrm{H}), 3.39(\mathrm{dd}, J=17.9,3.7,1 \mathrm{H}) ;{ }^{13} \mathrm{C} \mathrm{NMR}\left(125 \mathrm{MHz}, \mathrm{CDCl}_{3}\right) \delta$ 199.4, 198.5, 159.1, 136.8, 133.5, 133.1, 130.8, 129.6, 129.2, 128.8, 128.7, 128.4, 117.1, 55.5, 48.1, 44.2. All spectral data match those reported by Horspool and Khalaf. ${ }^{7}$

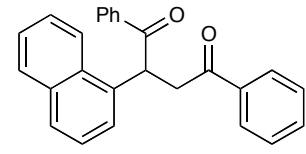

2-(Naphthalene-4-yl)-1,4-diphenylbutane-1,4-dione (7): Purified with 10\% ether/hexanes yielding $101 \mathrm{mg}(72 \%)$ of 7 as a white solid. $\mathrm{R}_{f}=0.40(40 / 60$ ether/hexanes); IR (film): 3059, 1681, $1598 \mathrm{~cm}^{-1} ;{ }^{1} \mathrm{H}$ NMR (500 MHz, $\left.\mathrm{CDCl}_{3}\right)$ 8.34-8.33 (m, 1H); 8.00-7.92 (m, 5H); 7.78-7.76 (m, 1H); 7.67-7.64 $(\mathrm{m}, 1 \mathrm{H}) ; 7.59-7.54(\mathrm{~m}, 2 \mathrm{H}) ; 7.46-7.41(\mathrm{~m}, 3 \mathrm{H}) ; 7.37-7.28(\mathrm{~m}, 4 \mathrm{H}) ; 6.12(\mathrm{dd}, \mathrm{J}=10.4,2.0 \mathrm{~Hz}$, $1 \mathrm{H}) ; 4.30(\mathrm{dd}, \mathrm{J}=18.1,10.6 \mathrm{~Hz}, 1 \mathrm{H}) ; 3.28(\mathrm{dd}, \mathrm{J}=18.1,2.3 \mathrm{~Hz}, 1 \mathrm{H}) ;{ }^{13} \mathrm{C} \mathrm{NMR}(125 \mathrm{MHz}$ $\left.\mathrm{CDCl}_{3}\right) \delta 199.4,198.4,136.7,136.5,135.3,134.7,133.5,133.1,130.8,129.6,129.0,128.8$, $128.7,128.5,128.3,127.3,126.3,126.0,122.7,44.5,43.0$; LRMS (electrospray): Mass calculated for $\mathrm{C}_{26} \mathrm{H}_{20} \mathrm{O}_{2}[\mathrm{M}+\mathrm{Na}]^{+}$, 387.2. Found 387.0.

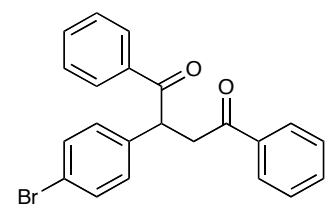

2-(4-Bromophenyl)-1,4-diphenylbutane-1,4-dione (8): Purified with $10 \%$ ether/hexanes, yielding $100 \mathrm{mg}(66 \%)$ of $\mathbf{8}$ as a colorless oil. $\mathbf{R}_{f}=$ 0.50 (40/60 ether/hexanes); IR (film) 3060, 2922, 1681, $1596 \mathrm{~cm}^{-1} ;{ }^{1} \mathrm{H}$ NMR (500 MHz, $\left.\mathrm{CDCl}_{3}\right) \delta 8.02-7.97(\mathrm{~m}, 4 \mathrm{H}) ; 7.58-7.50(\mathrm{~m}, 2 \mathrm{H}) ; 7.47-$ $7.40(\mathrm{~m}, 4 \mathrm{H}) ; 7.32-7.27(\mathrm{~m}, 4 \mathrm{H}) ; 5.32(\mathrm{dd}, \mathrm{J}=9.7,4.0 \mathrm{~Hz}, 1 \mathrm{H}) ; 4.17(\mathrm{dd}, \mathrm{J}$

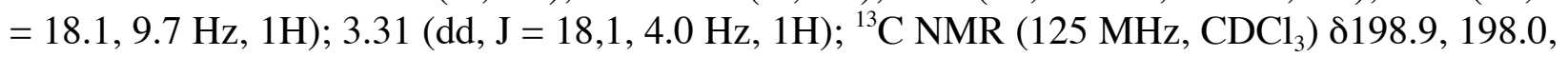
137.3, 136.5, 136.4, 133.6, 133.6, 133.4, 129.8, 129.6, 129.1, 128.9, 128.8, 128.4, 48.2, 43.9. LRMS (electrospray): Mass calculated for $\mathrm{C}_{22} \mathrm{H}_{17} \mathrm{BrO}_{2}[\mathrm{M}+\mathrm{Na}]^{+}, 415.0$. Found 416.8. 


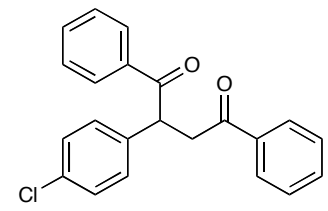

2-(4-Chlorophenyl)-1,4-diphenylbutane-1,4-dione (9): Purified with 10\% ether/hexanes yielding $99 \mathrm{mg}(74 \%)$ of 9 as a white solid. $\mathrm{R}_{f}=0.50(40 / 60$ ether/hexanes); IR (film): 3054, 2986, 1682, $1597 \mathrm{~cm}^{-1} ;{ }^{1} \mathrm{H}$ NMR $(500 \mathrm{MHz}$, $\left.\mathrm{CDCl}_{3}\right) \delta$ 8.04-7.97 (m, 4H), 7.58-7.55 (m, $\left.1 \mathrm{H}\right), 7.53-7.50(\mathrm{~m}, 1 \mathrm{H})$, 7.47$7.40(\mathrm{~m}, 4 \mathrm{H}), 7.32-7.27(\mathrm{~m}, 4 \mathrm{H}), 5.32(\mathrm{dd}, \mathrm{J}=9.9,3.8 \mathrm{~Hz}, 1 \mathrm{H}), 4.17(\mathrm{dd}, \mathrm{J}$ $=17.9,9.9 \mathrm{~Hz}, 1 \mathrm{H}), 3.31(\mathrm{dd}, \mathrm{J}=17.9,3.8 \mathrm{~Hz}, 1 \mathrm{H}) ;{ }^{13} \mathrm{C} \mathrm{NMR}\left(100 \mathrm{MHz}, \mathrm{CDCl}_{3}\right) \delta$ 198.8, 197.9, 137.3, 136.5, 136.4, 133.6, 133.5, 133.3, 129.8, 129.6, 129.1, 128.8, 128.4, 48.2, 44.0; LRMS (electrospray): Mass calculated for $\mathrm{C}_{22} \mathrm{H}_{17} \mathrm{ClO}_{2}[\mathrm{M}+\mathrm{Na}]^{+}, 371.1$. Found 371.0.

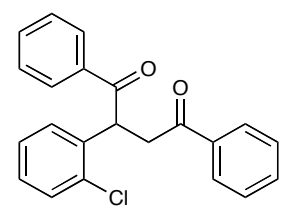

2-(2-Chlorophenyl)-1,4-diphenylbutane-1,4-dione (10): Purified with $10 \%$ ether/hexanes yielding $66 \mathrm{mg}(68 \%)$ of $\mathbf{1 0}$ as a colorless oil. $\mathrm{R}=0.48(40 / 60$ ether/hexanes); IR (film) 3054, 1681, $1597 \mathrm{~cm}^{-1}$; ${ }^{1} \mathrm{H}$ NMR (500 MHz, $\mathrm{CDCl}_{3}$ ) ठ 8.02-8.00 (m, 4H), 7.59-7.39 (m, 7H), 7.22-7.16 (m, 3H), $5.79(\mathrm{dd}, \mathrm{J}=10.9$, $4.0 \mathrm{~Hz}, 1 \mathrm{H}), 4.10(\mathrm{dd}, J=17.7,10 \mathrm{~Hz}, 1 \mathrm{H}), 3.23(\mathrm{dd}, J=17.7,3.7 \mathrm{~Hz}, 1 \mathrm{H})$

${ }^{13} \mathrm{C}$ NMR $\left(125 \mathrm{MHz}, \mathrm{CDCl}_{3}\right) \delta 198.8,197.8,136.6,136.3,133.5,133.4,130.5,129.3,129.1$, 128.9, 128.8, 128.8, 128.4, 127.8, 45.1, 42.5; LRMS (electrospray): Mass calculated for $\mathrm{C}_{22} \mathrm{H}_{17} \mathrm{ClO}_{2}[\mathrm{M}+\mathrm{Na}]^{+}$371.1. Found 372.0.

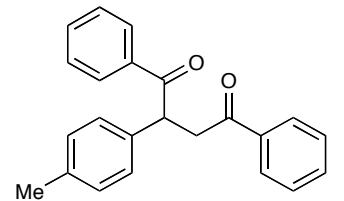

2-(4-Methylphenyl)-1,4-diphenylbutane-1-4-dione (11): Purified with $10 \%$ ether/ hexanes yielding $118 \mathrm{mg}(84 \%)$ of $\mathbf{1 1}$ as a white solid. $\mathrm{R}_{f}=0.47$ (40/60 ether hexanes); IR (film) 3054, 1681, $1597 \mathrm{~cm}^{-1} ;{ }^{1} \mathrm{H}$ NMR (500

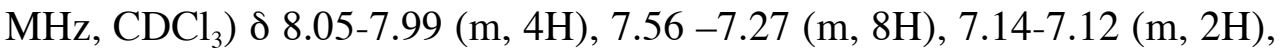
$5.32(\mathrm{dd}, J=10.1,3.5 \mathrm{~Hz}, 1 \mathrm{H}), 4.24(\mathrm{dd}, J=18.0,3.6) 3.31(\mathrm{dd}, J=18.0$, $3.5 \mathrm{~Hz}), 2.30$ (s, 3H); ${ }^{13} \mathrm{C} \mathrm{NMR}\left(125 \mathrm{MHz} \mathrm{CDCl}_{3}\right) \delta 199.3,198.4,137.3,136.7,135.8,133.5$, 133.1, 130.5, 129.2, 128.8, 128.7, 128.4, 128.3, 48.5, 44.1, 21.3; LRMS (electrospray): Mass calculated for $\mathrm{C}_{23} \mathrm{H}_{20} \mathrm{O}_{2}[\mathrm{M}+\mathrm{Na}]^{+} 351.1$. Found 351.0. All spectral data match those reported by Nagarajan and Shechter. ${ }^{8}$

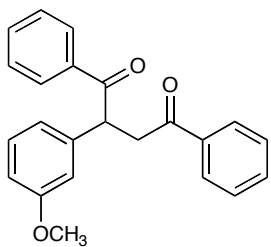

2-(3-Methoxyphenyl)-1,4-diphenylbutane-1,4-dione (12): Purified with $15 \%$ ether/hexanes yielding $99 \mathrm{mg}(75 \%)$ of $\mathbf{1 2}$ as a yellow oil. $\mathbf{R}_{f}=0.38(40 / 60$ ether/hexanes); IR (film) 2922, 1680, 1597, $1485 \mathrm{~cm}^{-1} ;{ }^{1} \mathrm{H}$ NMR $(500 \mathrm{MHz}$,

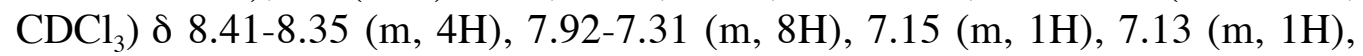
$5.66(\mathrm{dd}, J=10.3,3.1 \mathrm{~Hz}, 1 \mathrm{H}), 4.57(\mathrm{dd}, J=17.9,9.5 \mathrm{~Hz}, 1 \mathrm{H}), 4.14(\mathrm{~s}, 3 \mathrm{H})$, $3.66(\mathrm{dd}, J=17.9,3.7 \mathrm{~Hz}, 1 \mathrm{H}) ;{ }^{13} \mathrm{C}$ NMR $\left(125 \mathrm{MHz}, \mathrm{CDCl}_{3}\right) \delta 198.9,198.3$, $160.3,140.4,136.7,133.5,130.5,129.2,128.8,128.7,126.4,114.1,112.9,55.5,48.9,44.1$; LRMS (electrospray): Mass calculated for $\mathrm{C}_{23} \mathrm{H}_{20} \mathrm{O}_{3}[\mathrm{M}+\mathrm{Na}]^{+}$367.1. Found 367.0.

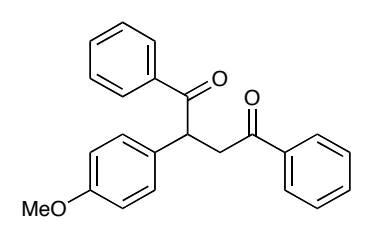

2-(4-Methoxyphenyl)-1,4-diphenylbutane-1,4-dione (13): Purified with $15 \%$ ether hexanes yielding $102 \mathrm{mg}(77 \%)$ of $\mathbf{1 3}$ as a yellow solid. $\mathbf{R}_{f}=$ 0.35 (40/60 ether/hexanes); IR (film) $1678,1596 \mathrm{~cm}^{-1} ;{ }^{1} \mathrm{H}$ NMR (500 
$\left.\mathrm{MHz}, \mathrm{CDCl}_{3}\right) \delta$ 8.01-7.99 (m, 4H), 7.53-7.35 (m, 8H), $7.24(\mathrm{~m}, 2 \mathrm{H}), 5.27(\mathrm{dd}, J=10.0,4.0$ $\mathrm{Hz}, 1 \mathrm{H}), 4.17(\mathrm{dd}, J=18.0,9.7 \mathrm{~Hz}, 1 \mathrm{H}), 3.72(\mathrm{~s}, 3 \mathrm{H}), 3.25(\mathrm{dd}, J=18.3,3.6,1 \mathrm{H}) ;{ }^{13} \mathrm{C} \mathrm{NMR}$ $\left(125 \mathrm{MHz} \mathrm{CDCl}_{3}\right) \delta 199.4,198.5,159.1,136.8,133.5,133.1,130.8,129.6,129.2,128.8,128.4$, 127.8, 114.9, 55.5, 48.1, 44.1; LRMS (electrospray): Mass calculated for $\mathrm{C}_{23} \mathrm{H}_{20} \mathrm{O}_{3}[\mathrm{M}+\mathrm{Na}]^{+}$ 367.1. Found 367.0. All other spectral data match those reported by Nagarajan and Shechter. ${ }^{8}$

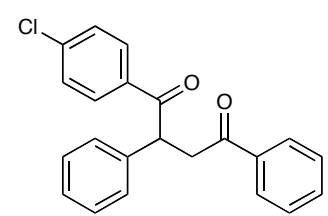

1-(4-chlorophenyl)-2-4-diphenylbutane-1,4-dione (15): Purified with $10 \%$ ether/hexanes yielding $67 \mathrm{mg}(82 \%)$ of $\mathbf{1 5}$ as a clear oil. $\mathrm{R}_{f}=0.48$ (40/60 ether/hexanes); IR (film): 3061, 2930, 1680, $1588 \mathrm{~cm}^{-1} ;{ }^{1} \mathrm{H}$ NMR $\left(400 \mathrm{MHz} \mathrm{CDCl}_{3}\right) \delta$ 7.97-7.93 (m, 4H), $7.55(\mathrm{~m}, 2 \mathrm{H}), 7.47-7.25(\mathrm{~m}, 8 \mathrm{H})$, $5.25(\mathrm{dd}, J=10.4,3.7 \mathrm{~Hz}, 1 \mathrm{H}), 4.23(\mathrm{dd}, J=18.3,10.4 \mathrm{~Hz}, 1 \mathrm{H}), 3.30(\mathrm{dd}$, $J=17.7,3.7 \mathrm{~Hz}, 1 \mathrm{H}) ;{ }^{13} \mathrm{C} \mathrm{NMR}\left(125 \mathrm{MHz} \mathrm{CDCl}_{3}\right) \delta 198.3,198.0,139.6,138.5,136.5,135.0$, 133.6, 130.6, 129.6, 129.1, 128.9, 128.4, 127.8, 49.0, 44.1; LRMS (electrospray): Mass calculated for $\mathrm{C}_{22} \mathrm{H}_{17} \mathrm{ClO}_{2}[\mathrm{M}+\mathrm{Na}]^{+}, 371.1$. Found 371.0. All spectral data match those reported by Stetter and Schreckenberg. ${ }^{9}$

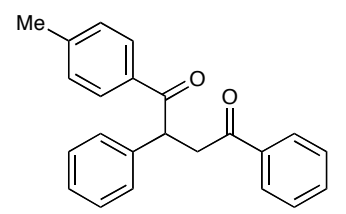

2,4-diphenyl-1-p-tolylbutane-1,4-dione (16): Purified with 10\% ether/hexanes yielding $84 \mathrm{mg}(70 \%)$ of $\mathbf{1 6}$ as a white solid. $\mathrm{R}_{f}=0.42$ (40/60 ether/hexanes); IR (film): 2927, 1678, 1596, $1511 \mathrm{~cm}^{-1} ;{ }^{1} \mathrm{H}$ NMR $\left(500 \mathrm{MHz}, \mathrm{CDCl}_{3}\right) \delta 8.15-8.08(\mathrm{~m}, 4 \mathrm{H}), 7.66-7.22(\mathrm{~m}, 10 \mathrm{H}), 5.41(\mathrm{dd}, J=$ 9.9, $3.6 \mathrm{~Hz}, 1 \mathrm{H}), 4.29(\mathrm{dd}, J=17.9,9.9 \mathrm{~Hz}, 1 \mathrm{H}), 3.39(\mathrm{dd}, J=17.9,3.5$ $\mathrm{Hz}, 1 \mathrm{H}), 2.40(\mathrm{~s}, 3 \mathrm{H}) ;{ }^{13} \mathrm{C}$ NMR $\left(125 \mathrm{MHz}, \mathrm{CDCl}_{3}\right) \delta 199.3,198.4,137.4,136.7,135.8,133.5$, $133.1,130.2$, 129.2, 128.8, 128.7, 128.4, 128.3, 48.6, 44.2, 21.3; All spectral data match those reported by Zdrojweski and Jonczyk. ${ }^{10}$

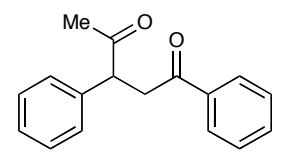

1,3-Diphenylpentane-1,4-dione (17): Purified with $10 \%$ ether/hexanes yielding $49 \mathrm{mg}(70 \%)$ of $\mathbf{1 7}$ as a pale yellow oil. $\mathrm{R}_{f}=0.42(40 / 60$ ether/hexanes); IR (film): 3060, 3028, 2918, 1716, $1684 \mathrm{~cm}^{-1} ;{ }^{1} \mathrm{H}$ NMR (500 $\left.\mathrm{MHz} \mathrm{CDCl}_{3}\right) \delta$ 7.97-7.95 (m, 2H); 7.58-7.52 (m, 1H); 7.55-7.42 (m, 2H); 7.37-7.34 (m, 2H); 7.30-7.29 (m, 3H); 4.45-4.42 (dd, $J=10.1,3.7 \mathrm{~Hz}, 1 \mathrm{H})$; 4.05-3.99 (dd, J = $17.9,10.1 \mathrm{~Hz}, 1 \mathrm{H}) ; 3.16-3.12(\mathrm{dd}, J=17.9,3.7 \mathrm{~Hz}, 1 \mathrm{H}) ; 2.22(\mathrm{~s}, 3 \mathrm{H}) ;{ }^{13} \mathrm{C} \mathrm{NMR}(125 \mathrm{MHz}$, $\left.\mathrm{CDCl}_{3}\right) \delta 207.47,198.4,138.2,136.7,133.5,129.4,128.8,128.6,128.3,127.9,54.1,42.5,29.4$; LRMS (electrospray): Mass calculated for $\mathrm{C}_{17} \mathrm{H}_{16} \mathrm{O}_{2}[\mathrm{M}+\mathrm{Na}]^{+}, 272.3$. Found 275.0. All spectral data match those reported by Hegedus and Perry. ${ }^{11}$

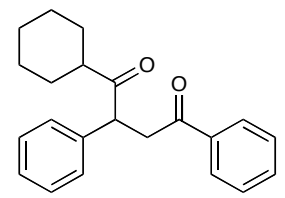

1-Cyclohexyl-2-4-diphenyl-1-4-dione (18): Purified with $10 \%$ ether/hexanes yielding $40 \mathrm{mg}(63 \%)$ of $\mathbf{1 8}$ as a clear oil. $\mathrm{R}_{f}=0.48$ (40/60 ether/hexanes); IR (film): 3059, 2929, 1721, 1664, 1605, $1576 \mathrm{~cm}^{-1} ;{ }^{1} \mathrm{H}$ NMR $\left(500 \mathrm{MHz}, \mathrm{CDCl}_{3}\right)$ d 8.06-7.97 (m, 4H), 7.68-7.29 (m, 6H), $4.60(\mathrm{dd}, J=10.1,2.9 \mathrm{~Hz}, 1 \mathrm{H}), 4.01$ (dd, $J=19.8,10.4 \mathrm{~Hz}, 1 \mathrm{H}), 3.09$ (dd, $J=17.9,3.11 \mathrm{~Hz}, 1 \mathrm{H}), 2.53(\mathrm{~m}, 1 \mathrm{H}), 2.15(\mathrm{~m}, 1 \mathrm{H}), 1.81$ $-1.09(\mathrm{~m}, 9 \mathrm{H}) ;{ }^{13} \mathrm{C} \mathrm{NMR}\left(125 \mathrm{MHz}, \mathrm{CDCl}_{3}\right) \delta 212.5,198.5,138.5,133.4,130.8,129.3,129.2$, $128.9,128.4,127.6,52.0,50.8,43.0,29.7,28.6,26.2,26.1$; All spectral data match those reported by Yus and coworkers. ${ }^{12}$ 


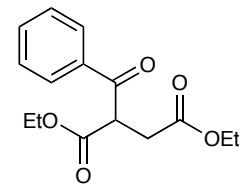

Diethyl 2-benzoylsuccinate (19): Purified with $10 \%$ ether/hexanes yielding 50 $\mathrm{mg}(65 \%)$ of 19 as a pale yellow oil. $\mathrm{R}_{f}=0.35$ (40/60 ether/hexanes); IR (film) $3057,2985,1735,1688,1598 \mathrm{~cm}^{-1} ;{ }^{1} \mathrm{H}$ NMR $\left(500 \mathrm{MHz}, \mathrm{CDCl}_{3}\right) \delta 8.07-8.06(\mathrm{~m}$, $2 \mathrm{H}), 7.64-7.61(\mathrm{~m}, 1 \mathrm{H}), 7.53-7.50(\mathrm{~m}, 2 \mathrm{H}) ; 4.89(\mathrm{dd}, J=7.3,7.3 \mathrm{~Hz}, 1 \mathrm{H}), 4.16$ $(\mathrm{m}, J=7.0 \mathrm{~Hz}, 4 \mathrm{H}), 3.12(\mathrm{dd}, J=17.4,7.7 \mathrm{~Hz}, 1 \mathrm{H}), 3.04(\mathrm{dd}, J=17.4,6.6 \mathrm{~Hz}, 1 \mathrm{H}), 1.25(\mathrm{t}, J=$ $7.1 \mathrm{~Hz}, 3 \mathrm{H}), 1.18(\mathrm{t}, J=7.1 \mathrm{~Hz}, 3 \mathrm{H}) ;{ }^{13} \mathrm{C} \mathrm{NMR}\left(125 \mathrm{MHz}, \mathrm{CDCl}_{3}\right) \delta 194.5,171.5,169.0,136.2$, 133.9, 129.1, 129.0, 62.1, 61.3, 49.9, 33.5, 14.3, 14.2; LRMS (electrospray): Mass calculated for $\mathrm{C}_{15} \mathrm{H}_{18} \mathrm{O}_{5}[\mathrm{M}+\mathrm{Na}]^{+}$301.1. Found 301.0. All spectral data match those reported by Saigo and coworkers. ${ }^{13}$

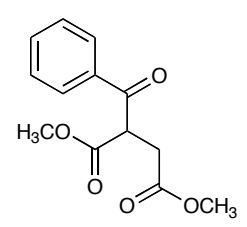

Dimethyl 2-benzoylsuccinate (20): Purified with $15 \%$ ethyl acetate/ hexanes yielding $50 \mathrm{mg}(72 \%)$ of $\mathbf{2 0}$ as a clear oil. $\mathrm{R}_{f}=0.35$ (50/50 ethyl acetate/hexanes); IR (film): 3002, 2945, 1738, 1684, 1596, $1581 \mathrm{~cm}^{-1}$; ${ }^{1} \mathrm{H}$ NMR $\left(500 \mathrm{MHz}, \mathrm{CDCl}_{3}\right) \delta 8.04(\mathrm{~d}, J=7.7 \mathrm{~Hz}, 2 \mathrm{H}), 7.62-7.52(\mathrm{~m}, 3 \mathrm{H}), 4.91(\mathrm{dd}, J=$ 7.3, 7.3 Hz, 1H), $3.69(\mathrm{~s}, 6 \mathrm{H}), 3.12(\mathrm{dd}, J=8.8,7.7 \mathrm{~Hz}, 1 \mathrm{H}), 3.10(\mathrm{dd}, J=8.8$, $7.9 \mathrm{~Hz}, 1 \mathrm{H}) ;{ }^{13} \mathrm{C}$ NMR $\left(125 \mathrm{MHz}, \mathrm{CDCl}_{3}\right) \delta$ 194.3, 171.9, 169.4, 136.1, 134.0, 129.2, 129.0, 53.1, 52.4, 49.5, 33.3; LRMS (electrospray): Mass calculated for $\mathrm{C}_{13} \mathrm{H}_{14} \mathrm{O}_{5}[\mathrm{M}+\mathrm{Na}]^{+}, 273.1$. Found 272.9. All spectral data match those reported by Kawenoki and co-workers. ${ }^{14}$

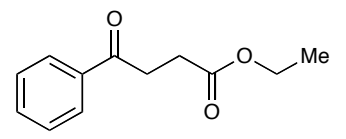

Ethyl 4-oxo-4-phenylbutanoate (21): Purified with 10\% ethyl acetate/hexanes yielding $40 \mathrm{mg}(72 \%)$ of $\mathbf{2 1}$ as a clear oil. $\mathrm{R}_{f}=0.32$; IR: 2951, 1732, 1595, $1447 \mathrm{~cm}^{-1} ;{ }^{1} \mathrm{H}$ NMR $\left(500 \mathrm{MHz}, \mathrm{CDCl}_{3}\right) \delta$ 8.00-7.98 (m, $2 \mathrm{H}), 7.58-7.45(\mathrm{~m}, 3 \mathrm{H}), 4.14(\mathrm{q}, J=6.7,2 \mathrm{H}), 3.33(\mathrm{t}, J=6.7,2 \mathrm{H}), 2.75(\mathrm{t}, J=6.1,2 \mathrm{H}), 1.27(\mathrm{t}$, $J=6.7,3 \mathrm{H}) ;{ }^{13} \mathrm{C}\left(125 \mathrm{MHz}, \mathrm{CDCl}_{3}\right) \delta 198.4,172.5,135.3,133.0,128.9,128.0,61.5,33.3,28.1$, 13.8. All spectral data match those reported by Rieke and coworkers. ${ }^{15}$

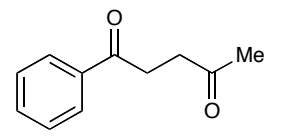

1-Phenylpentane-1,4-dione (22): Purified with $15 \%$ ethyl acetate/hexanes yielding $36 \mathrm{mg}(75 \%)$ of $\mathbf{2 2}$ as a yellow oil. $\mathrm{R}_{f}=0.35$ (50/50 ethyl acetate/hexanes); IR (film) 2975, 2931, 1716, 1685; 1H (500 MHz, CDCl3) $\delta$ $8.00(\mathrm{~d}, J=7.3,2 \mathrm{H}), 7.50-7.47(\mathrm{~m}, 3 \mathrm{H}), 3.31(\mathrm{t}, J=6.6,2 \mathrm{H}), 2.92(\mathrm{t}, J=6.4,2 \mathrm{H}), 2.29(\mathrm{~s}, 3 \mathrm{H})$; ${ }^{13} \mathrm{C}$ NMR $\left(125 \mathrm{MHz}, \mathrm{CDCl}_{3}\right) \delta$ 207.6, 198.8, 136.9, 133.4, 128.8, 128.3, 37.3, 32.7, 30.4; All spectral data match those reported by Shibata and coworkers. ${ }^{16}$

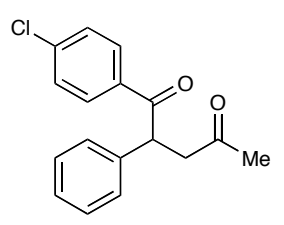

1-(4-chlorophenyl)-2-phenylpentane-1,4-dione (23): Purified using a gradient starting with $5 \%$ ether/hexanes yielding $55 \mathrm{mg}(40 \%)$ of $\mathbf{2 3}$ as a yellow oil. $\mathrm{R}_{f}=0.27$ (40\% ether/hexanes); IR (film) 3062, 2920, 1715, 1588 $\mathrm{cm}^{-1}$; ${ }^{1} \mathrm{H}$ NMR $\left(500 \mathrm{MHz}, \mathrm{CDCl}_{3}\right) \delta$ 7.93-7.92 (m, 2H), 7.38-7.25 (m, 7H), $5.07(\mathrm{dd}, J=9.8,3.7 \mathrm{~Hz}, 1 \mathrm{H}), 3.60(\mathrm{dd}, J=18.3,9.8 \mathrm{~Hz}, 1 \mathrm{H}), 2.75(\mathrm{dd}, J=$ 18.3, 3.7 Hz, 1H), 2.20 (s, 3H); ${ }^{13} \mathrm{C}$ NMR $\left(125 \mathrm{MHz}, \mathrm{CDCl}_{3}\right) \delta$ 207.0, 198.3, 139.6, 138.4, 134.8, $130.6,129.6,129.1,128.5,128.3,128.0,127.7,49.05,48.3,30.2$. All spectral data match that of Stetter and Scheckenberg. ${ }^{8}$ 


\section{General Procedure for Thiazolium-Catalyzed Furan Synthesis:}

A screw-capped test tube was charged with the thiazolium salt ( 0.20 equiv) and placed under nitrogen. Acylsilane (1.0 equiv) in THF $(0.25 \mathrm{~mL})$ was added by syringe to the test tube followed by the addition of $\mathrm{DBU}\left(0.30\right.$ equiv). The reaction mixture was heated to $70^{\circ} \mathrm{C}$ after which the chalcone (1.0 equiv) in THF $(0.25 \mathrm{~mL})$ was added by syringe followed by the addition of 2-propanol (4.0 equiv). The reaction was allowed to stir at $70^{\circ} \mathrm{C}$ for 24 hours or until completion as determined by TLC ( $40 \%$ ether/hexanes). To the solution acetic acid (1.0 equiv) was added. The reaction mixture remained heating at $70^{\circ} \mathrm{C}$ for an additional 12 to 24 hours or until consumption of chalcone produx as determined by TLC ( $40 \%$ ether/hexanes). Upon cooling to room temperature, the reaction mixture was diluted with water $(30 \mathrm{~mL})$ and washed with dichloromethane $(30 \mathrm{~mL})$. The aqueous layer was washed with dichloromethane $(3 \mathrm{X} 30$ $\mathrm{mL}$ ), dried over sodium sulfate, filtered, and concentrated in vacuo. The resulting residue was purified by flash column chromatography on silica gel.

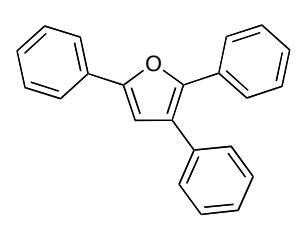

2, 3, 5 triphenylfuran (25): Purified with 5-10\% ether/hexanes yielding 65 $\mathrm{mg}(81 \%)$ of $\mathbf{2 5}$ as a white solid. $\mathrm{R}_{f}=0.72$ (25\% ethyl acetate/hexanes); IR (film) 3055, 2967, 2357, 1493, 1261, 1074, $801 \mathrm{~cm}^{-1}$; ${ }^{1} \mathrm{H}$ NMR (400 MHz, $\left.\mathrm{CDCl}_{3}\right) \delta$ 7.89-7.19 (m, 15H), $6.89(\mathrm{~s}, 1 \mathrm{H}) ;{ }^{13} \mathrm{C} \mathrm{NMR}(125 \mathrm{MHz}) \delta 153.4$, $148.2,134.6,131.5,130.9,128.9,128.8,128.5,128.1,127.1,126.9,124.8$, 124.1, 109.9; All other spectral values are identical to those reported by Fürstner and Jumbam. ${ }^{17}$

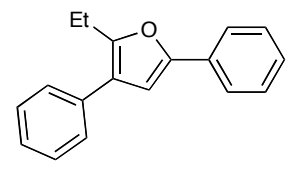

2-ethyl-3,5-diphenylfuran (26): Purified with 5-10\% ether/hexanes yielding $68 \mathrm{mg}(82 \%)$ of $\mathbf{2 6}$ as a clear oil. $\mathrm{R}_{f}=0.75$ (25\% ethyl acetate/hexanes); ${ }^{1} \mathrm{H}$ NMR $\left(500 \mathrm{MHz}, \mathrm{CDCl}_{3}\right) \delta 7.70-7.14(\mathrm{~m}, 10 \mathrm{H}), 6.73(\mathrm{~s}, 1 \mathrm{H}), 2.92(\mathrm{q}, J=7.7$ $\mathrm{Hz}, 2 \mathrm{H}), 1.37(\mathrm{t}, J=7.6 \mathrm{~Hz} 3 \mathrm{H})$; All spectral values are identical to those reported by Luh and coworkers. ${ }^{18}$

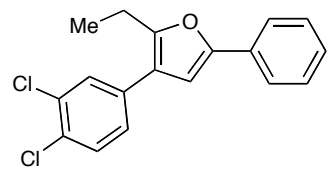

3-(3,4-dichlorophenyl)-2-ethyl-5-phenylfuran (27): Purified with $10 \%$ ether/hexanes to yield $76 \mathrm{mg}(81 \%)$ of 27 as a clear oil. $\mathrm{R}_{f}=0.68 \quad(30 \%$ ethyl acetate/hexanes); IR (film) 3062, 2960, 1701, 16661, 1588, 1257, 1110, 1041, $797 \mathrm{~cm}^{-1} ;{ }^{1} \mathrm{H}$ NMR $\left(500 \mathrm{MHz} \mathrm{CDCl}_{3}\right) \delta 7.93-7.16(\mathrm{~m}, 8 \mathrm{H})$, 6.67(s, 1H), 2.76 (q, J=7.3), 1.26 (t, 7.3); ${ }^{13} \mathrm{C}$ NMR (125 MHz) $\delta 150.6,149.3,134.4,132.3$, $130.2,127.6,126.4,125.9,124.9,124.6,120.7,103.0,17.8,10.3$; Mass calculated (ESI) for $\left[\mathrm{C}_{18} \mathrm{H}_{15} \mathrm{Cl}_{2} \mathrm{O}+1\right] 318.4$; Found 318.1 .

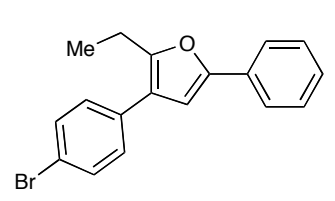

3-(4-bromophenyl-2-ethyl-5-phenylfuran (28): Purified with $10 \%$ ether in hexanes to yield $56 \mathrm{mg}(84 \%)$ of $\mathbf{2 8}$ as a yellow oil. $\mathrm{R}_{f}=0.75(30 \%$ ethyl acetate/hexanes); IR (film) 3062, 2959, 1702, 1582, 1485, 1404, 1258.1, 1043, $796 \mathrm{~cm}^{-1} ;{ }^{1} \mathrm{H}$ NMR (400 MHz, $\left.\mathrm{CDCl}_{3}\right) \delta$ 7.68-7.24 (m, 9H), $6.71(\mathrm{~s}, 1 \mathrm{H}), 2.83(\mathrm{q}, \mathrm{J}=7.3,2 \mathrm{H}), 1.34(\mathrm{t}, \mathrm{J}=7.3,3 \mathrm{H}) ;{ }^{13} \mathrm{C} \mathrm{NMR}(125 \mathrm{MHz})$ 


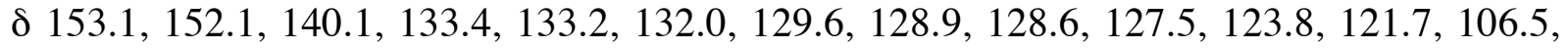
20.9, 13.3; Mass calculated for [ $\left.2 \mathrm{X} \mathrm{C}_{18} \mathrm{H}_{15} \mathrm{BrO}+\mathrm{Na}^{+}\right]$: 675.4; Found 675.3

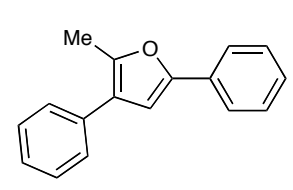

2-methyl-3, 5-diphenylfuran (29): Purified with $2 \%$ ethyl acetate/hexanes to give $66 \mathrm{mg}$ (74\%) of $\mathbf{2 9}$ as a yellow oil. IR (film) 3055, 2967, 1493, 1450, 1261, 1212, 1021, $759 \mathrm{~cm}^{-1} ;{ }^{1} \mathrm{H}$ NMR $\left(500 \mathrm{MHz}, \mathrm{CDCl}_{3}\right) \delta$ 7.73-7.18(m, $10 \mathrm{H}), 6.81(\mathrm{~s}, 1 \mathrm{H}), 2.49(\mathrm{~s}, 3 \mathrm{H}) ;{ }^{13} \mathrm{C}$ NMR $(125 \mathrm{MHz}) \delta 151.3,148.0,133.9$, 133.2, 130.8, 130.6, 129.4, 128.4, 127.8, 126.6, 126.5, 122.9, 118.9, 106.7, 13.0; All other spectral values are identical to those reported by Chong and co-workers. ${ }^{19}$

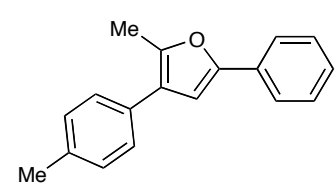

2-methyl-5-phenyl-4-p-furan (30): Purified with 5-10\% ether/hexanes yielding $75 \mathrm{mg}(83 \%)$ of $\mathbf{3 0}$ as a clear oil. $\mathrm{R}_{f}=0.72 \quad(25 \%$ ethyl acetate/hexanes); IR (film) 3053, 2958, 1704, 1656, 1576, 1253, 1188, $1041,825 \mathrm{~cm}^{-1} ;{ }^{1} \mathrm{H}$ NMR $\left(400 \mathrm{MHz}, \mathrm{CDCl}_{3}\right) \delta$ 7.71-7.55 (m, 4H), 7.42$7.12(\mathrm{~m}, 5 \mathrm{H}), 6.80(\mathrm{~s}, 1 \mathrm{H}), 2.54(\mathrm{~s}, 3 \mathrm{H}), 2.43(\mathrm{~s}, 3 \mathrm{H}) ;{ }^{13} \mathrm{C}$ NMR $(125 \mathrm{MHz}) \delta 156.0,153.9$, 137.4, 135.9, 130.7, 129.5, 129.0, 128.1, 127.6, 126.2, 124.0, 106.5, 21.5, 12.7; Mass calculated (ESI) for $\mathrm{C}_{18} \mathrm{H}_{6} \mathrm{O}\left[2 \mathrm{X} \mathrm{C}_{18} \mathrm{H}_{15} \mathrm{O}\right]$ 498.62; Found 499.1.

\section{General Procedure for Thiazolium-Catalyzed Pyrrole Synthesis:}

A screw-capped test tube was charged with the thiazolium salt $(14 \mathrm{mg}, 0.055 \mathrm{mmol}, 0.20$ equiv) in a nitrogen-filled drybox. The reaction vessel was removed from the box and placed under a positive pressure of nitrogen. Benzoyltrimethylsilane ( $100 \mathrm{mg}, 0.55 \mathrm{mmol}, 2.0$ equiv) in THF $(0.25 \mathrm{~mL})$ was added by syringe to the test tube followed by the addition of DBU $(13 \mathrm{~mL}, 0.083$ mmol, 0.30 equiv). The reaction mixture was heated to $70^{\circ} \mathrm{C}$ after which the chalcone $(0.275$ mmol, 1.0 equiv) in THF $(0.25 \mathrm{~mL})$ was added by syringe followed by the addition of 2 propanol ( $83 \mathrm{~mL}, 1.10 \mathrm{mmol}, 4.0$ equiv). The reaction was allowed to stir at $70^{\circ} \mathrm{C}$ for 12 hours or until completion as determined by TLC ( $40 \%$ ether/hexanes). To the solution amine $(0.825$ $\mathrm{mmol}, 3.0$ equiv) was added followed by addition of $p$-toluenesulfonic acid (104 $\mathrm{mg}, 0.55 \mathrm{mmol}$, 2.0 equiv) in ethanol $(0.5 \mathrm{~mL})$ and $4 \AA$ molecular sieves (spatula tip). The reaction mixture remained heating at $70^{\circ} \mathrm{C}$ for an additional 6 to 12 hours or until consumption of diketone as determined by TLC ( $25 \%$ ethyl acetate/ hexane). Upon cooling to room temperature, the reaction mixture was diluted with ethyl acetate $(30 \mathrm{~mL})$ and washed with water $(10 \mathrm{~mL})$. The aqueous layer was washed with ethyl acetate $(3 \times 30 \mathrm{~mL})$ and the combined the organic extracts were washed with brine $(10 \mathrm{~mL})$ and dried over sodium sulfate, filtered, and concentrated in vасио. The resulting residue was purified by flash column chromatography on silica gel.

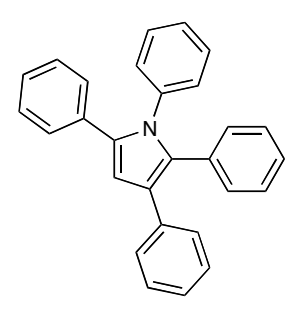

1,2,3,5-tetraphenyl-1- $\boldsymbol{H}$-pyrrole (31): Purified with $1 \%$ ethyl acetate/hexanes, yielding $67 \mathrm{mg}(66 \%)$ of $\mathbf{3 1}$ as a white solid. $\mathrm{R}_{f}=0.88(25 \%$ ethyl acetate/hexanes); mp: $200-201{ }^{\circ} \mathrm{C}$ (lit, $\left.200-202^{\circ} \mathrm{C}\right) .{ }^{1} \mathrm{H}$ NMR $(500 \mathrm{MHz}$, $\left.\mathrm{CDCl}_{3}\right) \delta$ 7.26- $6.97(\mathrm{~m}, 20 \mathrm{H}), 6.74(\mathrm{~s}, 1 \mathrm{H}) ;{ }^{13} \mathrm{C} \mathrm{NMR}\left(125 \mathrm{MHz}, \mathrm{CDCl}_{3}\right) \delta$ 139.0, 136.4, 133.2, 132.9, 132.4, 131.7, 129.4, 128.8, 128.7, 128.4, 128.2, 127.8, 127.4, 126.6, 125.7, 124.1, 123.7, 110.2; All other spectral information are similar to those reported by Stetter and coworkers. ${ }^{20}$ 

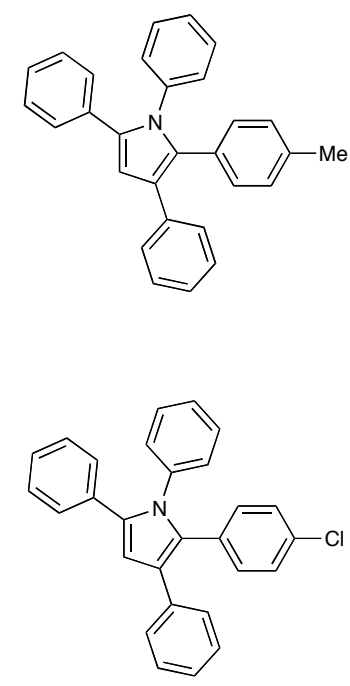

1,3,5-triphenyl-2-p-tolyl-1-H -pyrrole (32): Purified with $2 \%$ ethyl acetate/hexanes, yielding $75 \mathrm{mg}(71 \%)$ of 32as a clear oil. $\mathrm{R}_{f}=0.82(25 \%$ ethyl acetate/hexanes); ${ }^{1} \mathrm{H}$ NMR (500 MHz, $\mathrm{CDCl}_{3}$ ) $\delta$ 7.22- 7.05 (m, 19 $\mathrm{H}), 6.89(\mathrm{~s}, 1 \mathrm{H}), 2.36(\mathrm{~s}, 3 \mathrm{H}) ;{ }^{13} \mathrm{C} \mathrm{NMR}\left(125 \mathrm{MHz}, \mathrm{CDCl}_{3}\right) \delta 139.5$, 135.8, 135.0, 133.4, 133.0, 132.8, 132.2, 131.8, 130.0, 129.8, 129.5, 128.9, 128.7, 127.2, 126.1, 125.4, 122.7, 109.2, 20.3; MALDI: Mass (m/e) calculated for $\mathrm{C}_{29} \mathrm{H}_{23} \mathrm{~N}$ : 385.5. Found 385.8.

2-(4-chlorophenyl)-1,3,5-triphenyl-1- $\boldsymbol{H}$-pyrrole (33): Purified with $2 \%$ ethyl acetate/hexanes, yielding $68 \mathrm{mg}(61 \%)$ of $\mathbf{3 3}$ as a yellow solid. $\mathrm{R}_{f}=$ 0.61 (25\% ethyl acetate/hexanes); ${ }^{1} \mathrm{H}$ NMR $\left(500 \mathrm{MHz}, \mathrm{CDCl}_{3}\right) \delta$ 7.19-7.02 (m, 14H), 6.91-6.86 (m, 5H), $6.61(\mathrm{~s}, 1 \mathrm{H}),{ }^{13} \mathrm{C}$ NMR $\left(125 \mathrm{MHz}, \mathrm{CDCl}_{3}\right) \delta$ 138.8, 136.1, 135.5, 133.1, 132.9, 132.8, 131.6, 131.4, 130.9, 129.3, 129.0, $128.8,128.4,127.6,126.8,125.9,124.2,110.4$; All other spectral information are similar to those reported by Stetter and Schreckenberg. ${ }^{9}$

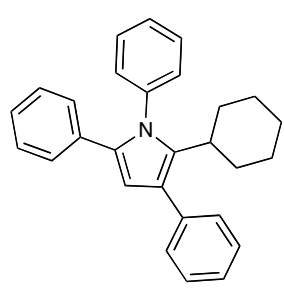

2-cyclohexyl-1, 3, 5-triphenyl-1H-pyrrole (34): Purified with 2\% ethyl acetate/hexanes yielding $71 \mathrm{mg}(69 \%)$ of $\mathbf{3 4}$ as a white solid. $\mathrm{R}_{f}=0.77(25 \%$ ethyl acetate/hexanes); mp: $162-163^{\circ} \mathrm{C}$. IR (film); ${ }^{1} \mathrm{H}$ NMR $(500 \mathrm{MHz}$, $\left.\mathrm{CDCl}_{3}\right)$ 8 7.50-7.38 (m, 8H), 7.10-7.08 (m, 7H), $6.40(\mathrm{~s}, 1 \mathrm{H}), 2.61(\mathrm{t}, J=12.1$, $1 \mathrm{H}), 1.73-0.90(\mathrm{~m}, 10 \mathrm{H}) ;{ }^{13} \mathrm{C}$ NMR $(125 \mathrm{MHz}) \delta 140.0,138.6,137.3,133.6$, 130.2, 130.0, 128.3, 128.2, 126.1, 125.9, 123.2, 111.5, 37.8, 33.5, 27.4, 26.1; MALDI: Mass (m/e) calculated for $\mathrm{C}_{28} \mathrm{H}_{27} \mathrm{~N}$ : 377.5. Found 377.2.

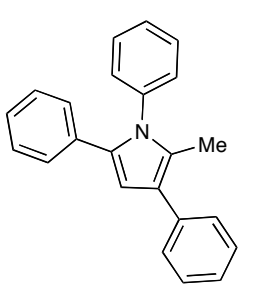

2-methyl-1, 3, 5-triphenyl-1 $\boldsymbol{H}$-pyrrole (35): Purified with 2\% ethyl acetate/hexanes, yielding $42 \mathrm{mg}(71 \%)$ of $\mathbf{3 5}$ as a white solid. $\mathrm{R}_{f}=0.58(25 \%$ ethyl acetate/hexanes); mp: $171-172^{\circ} \mathrm{C}$ (lit, $\left.173-175^{\circ} \mathrm{C}\right) .{ }^{1} \mathrm{H}$ NMR $(500 \mathrm{MHz}$, $\left.\mathrm{CDCl}_{3}\right) \delta$ 7.55-7.24 (m, 8H), 7.15-7.12 (m, 7H), $6.59(\mathrm{~s}, 1 \mathrm{H}), 2.27(\mathrm{~s}, 3 \mathrm{H}) ;{ }^{13} \mathrm{C}$ NMR $(125 \mathrm{MHz}) \delta 139.5,137.1,134.1,133.4,132.3,129.2,128.9,128.6,128.3$, 128.2, 128.1, 127.8, 126.1, 125.7, 122.9, 109.5, 12.7; All spectral data are similar to those report by Stetter and Schreckenberg. ${ }^{9}$
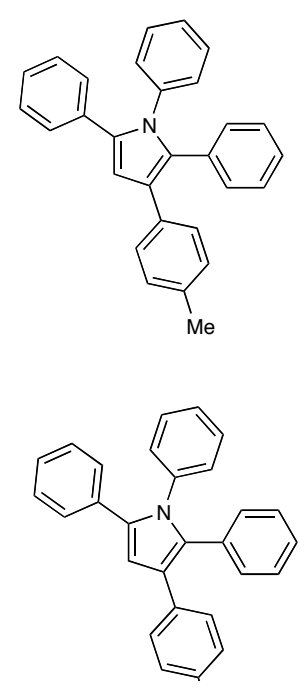

1,2,5-triphenyl-3-p-tolyl-1-H-pyrrole (36): Purified with $2 \%$ ethyl acetate/hexanes, yielding $74 \mathrm{mg}(70 \%)$ of $\mathbf{3 6}$ as a white solid. $\mathrm{R}_{f}=0.83(25 / 75$ ethyl acetate/hexanes); mp: $156-160^{\circ} \mathrm{C} .{ }^{1} \mathrm{H}$ NMR $\left(500 \mathrm{MHz}, \mathrm{CDCl}_{3}\right) \delta 7.22-$ $7.01(\mathrm{~m}, 19 \mathrm{H}), 6.73(\mathrm{~s}, 1 \mathrm{H}), 2.34(\mathrm{~s}, 3 \mathrm{H}) ;{ }^{13} \mathrm{C}$ NMR $\left(125 \mathrm{MHz}, \mathrm{CDCl}_{3}\right) \delta$ 139.1, 135.3, 134.9, 133.4, 133.2, 133.1, 131.8, 129.4, 129.2, 128.8, 128.7, 128.3, 128.2, 128.1, 127.2, 126.6, 123.7, 110.3, 21.4; MALDI: Mass (m/e) calculated for $\mathrm{C}_{29} \mathrm{H}_{23} \mathrm{~N}$ : 385.5. Found 385.4.

3-(-4-chlorophenyl)-1,2,5-triphenyl-1 $\boldsymbol{H}$-pyrrole (37): Purified with 2-3\% ethyl acetate/hexanes yielding $64 \mathrm{mg}(58 \%)$ of $\mathbf{3 7}$ as an oil. $\mathrm{R}_{f}=0.63(25 \%$ ethyl acetate/hexanes); ${ }^{1} \mathrm{H} \mathrm{NMR}\left(500 \mathrm{MHz}, \mathrm{CDCl}_{3}\right) \delta$ 7.17- $7.11(\mathrm{~m}, 15 \mathrm{H})$, 
7.03-6.98 (m, 4H), $6.68(\mathrm{~s}, 1 \mathrm{H}) ;{ }^{13} \mathrm{C}$ NMR $\left(100 \mathrm{MHz}, \mathrm{CDCl}_{3}\right) \delta$ 139.1, 136.2, 135.9, 131.6, 131.4, 129.8, 129.6, 129.6, 129.3, 128.8, 128.7, 128.5, 128.3, 127.5, 127.4, 126.7, 122.5, 109.9; MALDI: Mass (m/e) calculated for $\mathrm{C}_{28} \mathrm{H}_{20} \mathrm{ClN}$ : 405.9. Found 405.4.

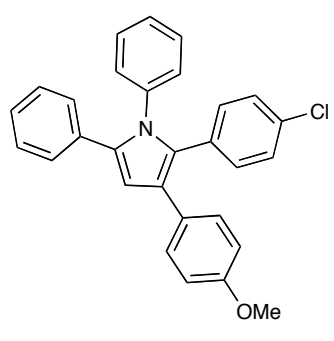

435.9. Found 435.3.

\section{2-(4-chlorophenyl)-3-(4-methoxyphenyl)-1,5-diphenyl-1 $H$-pyrrole} (38): Purified with 5\% ethyl acetate/hexanes, yielding $58 \mathrm{mg}(69 \%)$ of $\mathbf{3 8}$ as white solid. $\mathrm{R}_{f}=0.55$ (25\% ethyl acetate/hexanes); mp: $189-192^{\circ} \mathrm{C} .{ }^{1} \mathrm{H}$ NMR (500 MHz, $\left.\mathrm{CDCl}_{3}\right) \delta$ 7.21-7.10 (m, 14H), 7.09- $6.79(\mathrm{~m}, 4 \mathrm{H}), 6.64$ (s, $1 \mathrm{H}), 3.80$ (s, 3H); ${ }^{13} \mathrm{C}$ NMR $(125 \mathrm{MHz}) \delta 158.0,138.9,135.3,133.0$, $132.9,131.5,129.6,129.3,128.9,128.8,128.6,128.4,128.2,127.5,126.7$, 123.9, 114.0, 110.4, 55.4; MALDI: Mass (m/e) calculated for $\mathrm{C}_{29} \mathrm{H}_{22} \mathrm{ClNO}$ :

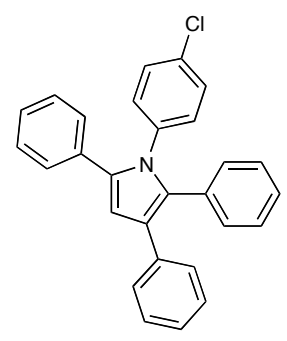

1-(4-Chlorophenyl)-2,3,5-triphenyl-1- $\boldsymbol{H}$-pyrrole (39): Purified with $2 \%$ ethyl acetate/hexanes, yielding $48 \mathrm{mg}(57 \%)$ of $\mathbf{3 9}$ as a yellow oil. $\mathbf{R}_{f}=0.61$ (25\% ethyl acetate/hexanes). ${ }^{1} \mathrm{H}$ NMR $\left(500 \mathrm{MHz}, \mathrm{CDCl}_{3}\right) \delta$ 7.24-6.91 (m, 19H), $6.71(\mathrm{~s}, 1 \mathrm{H}) ;{ }^{13} \mathrm{C}$ NMR $\left(125 \mathrm{MHz}, \mathrm{CDCl}_{3}\right) \delta 137.3,134.6,132.2,131.3$, $131.2,130.9,130.1,128.4,127.7,127.4,126.9,126.8,126.6,126.0,125.8$, 124.3, 122.3, 108.9; MALDI: Mass (m/e) calculated for $\mathrm{C}_{28} \mathrm{H}_{20} \mathrm{ClN}$ : 405.9. Found 405.1.

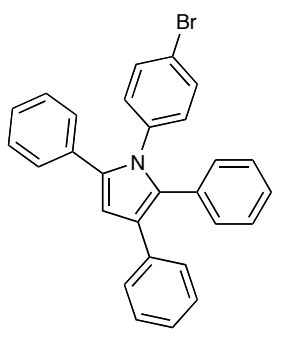

1-(4-bromophenyl)-2,3,5-triphenyl-1- $\boldsymbol{H}$-pyrrole (40): Purified with $2 \%$ ethyl acetate/hexanes, yielding $62 \mathrm{mg}(71 \%)$ of $\mathbf{4 0}$ as a white solid. $\mathrm{R}_{f}=0.58(25 \%$ ethyl acetate/hexanes); mp: $173-176^{\circ} \mathrm{C}$. IR (film) 3056, 3030, 1601, 1489, 1373; ${ }^{1} \mathrm{H}$ NMR $\left(400 \mathrm{MHz}, \mathrm{CDCl}_{3}\right) \delta$ 7.22-6.94 (m, 17H), $6.75(\mathrm{~d}, J=8.5 \mathrm{~Hz}$, 2H), $6.62(\mathrm{~s}, 1 \mathrm{H}) ;{ }^{13} \mathrm{C}$ NMR $\left(100 \mathrm{MHz}, \mathrm{CDCl}_{3}\right) \delta 138.1,136.0,134.9,132.8$, $132.5,131.9,131.7,130.7,128.9,128.4,128.3,127.4,126.8,125.8,121.1$, 110.6; MALDI: Mass (m/e) calculated for $\mathrm{C}_{28} \mathrm{H}_{20} \mathrm{BrN}$ : 450.4. Found 450.9 .

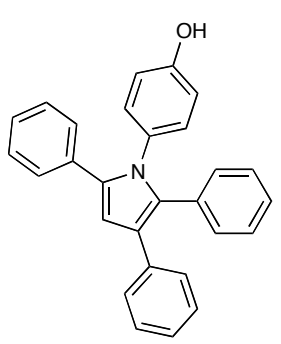

4-(2,3,5-triphenyl-1H-pyrrol-1yl)-phenol (41): Purified with $15 \%$ ethyl acetate/hexanes, yielding $65 \mathrm{mg}(61 \%)$ of $\mathbf{4 1}$ as oil. $\mathrm{R}_{f}=0.32$ (25\% ethyl acetate/hexanes); IR (film) 3394, 2962, 1598, 1513, $1261 \mathrm{~cm}^{-1}$; ${ }^{1} \mathrm{H}$ NMR (500 $\left.\mathrm{MHz}, \mathrm{CDCl}_{3}\right) \delta$ 7.27- $7.14(\mathrm{~m}, 15 \mathrm{H}), 6.86(\mathrm{~m}, 2 \mathrm{H}), 6.70(\mathrm{~s}, 1 \mathrm{H}), 6.63(\mathrm{~m}, 2 \mathrm{H})$, 4.71 (bs, $1 \mathrm{H}) ;{ }^{13} \mathrm{C}$ NMR $(125 \mathrm{MHz}) \delta 154.6,136.4,135.1,133.2,133.0,132.6$, $132.2,131.8,130.5,128.8,128.4,128.2,128.1,127.2,126.6,125.7,123.5$, 115.5, 109.9; MALDI: Mass (m/e) calculated for $\mathrm{C}_{28} \mathrm{H}_{21} \mathrm{NO}$ : 387.4. Found 387.1 .

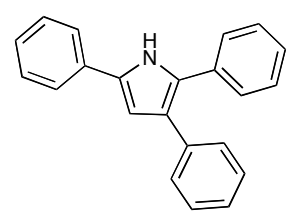

2,3,5-triphenyl-1 $\boldsymbol{H}$-pyrrole (42): Purified with $10 \%$ ethyl acetate/hexanes, yielding $50 \mathrm{mg}(62 \%)$ of $\mathbf{4 2}$ as a white solid. $\mathrm{R}_{f}=0.45$ (25\% ethyl acetate/hexanes); mp $134-135^{\circ} \mathrm{C}$ (lit. $135-137^{\circ} \mathrm{C}$ ). ${ }^{1} \mathrm{H}$ NMR $(500 \mathrm{MHz}$, 
$\left.\mathrm{CDCl}_{3}\right) \delta 8.36(\mathrm{~s}, 1 \mathrm{H}), 7.50(\mathrm{~m}, 2 \mathrm{H}), 7.38-7.10(\mathrm{~m}, 13 \mathrm{H}), 6.70(\mathrm{~s}, 1 \mathrm{H}) ;{ }^{13} \mathrm{C} \mathrm{NMR}(125 \mathrm{MHz})$ $\delta$ 136.5, 132.9, 132.1, 129.1, 128.9, 128.6, 128.4, 128.2, 127.7, 126.9, 126.0, 125.8, 122.9, 108.2; All other spectral information are similar to those reported by Fürstner and coworkers. ${ }^{21}$

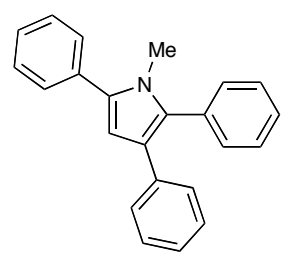

1-methyl-2,3,5-triphenyl-1- $\boldsymbol{H}$-pyrrole (43): Purified with 2\% ethyl acetate/hexanes, yielding $59 \mathrm{mg}(70 \%)$ of $\mathbf{4 3}$ as a white solid. $\mathbf{R}_{f}=0.73$ (25\% ethyl acetate/hexanes); Mp: $181-183^{\circ} \mathrm{C}$ (lit. $\left.{ }^{1} 178-179^{\circ} \mathrm{C}\right) .{ }^{1} \mathrm{H}$ NMR $(500$ $\left.\mathrm{MHz}, \mathrm{CDCl}_{3}\right) \delta$ 7.53-7.19 (m, $\left.15 \mathrm{H}\right), 6.51(\mathrm{~s}, 1 \mathrm{H}), 3.50$ (s, 3H); ${ }^{13} \mathrm{C}$ NMR $\left(125 \mathrm{MHz}, \mathrm{CDCl}_{3}\right) \delta 136.6,135.9,133.6,131.3,130.2,129.1,128.8,128.3$, 128.1, 127.8, 127.2, 125.4, 122.0, 109.0, 34.0; All spectral information are similar to those reported by Horspool and coworkers. ${ }^{22}$

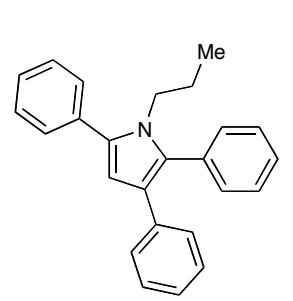

2,3,5-triphenyl-1-propyl-1-H-pyrrole (44): Purified with 1-3\% ethyl acetate/hexanes, yielding $76 \mathrm{mg}(82 \%)$ of $\mathbf{4 4}$ as a white solid. $\mathrm{R}_{f}=0.72(25 \%$ ethyl acetate/hexanes); mp: $123-124^{\circ} \mathrm{C} .{ }^{1} \mathrm{H}$ NMR $\left(500 \mathrm{MHz}, \mathrm{CDCl}_{3}\right) \delta$ 7.56$7.37(\mathrm{~m}, 9 \mathrm{H}), 7.23-7.11(\mathrm{~m}, 6 \mathrm{H}), 6.51(\mathrm{~s}, 1 \mathrm{H}), 3.93(\mathrm{t}, J=7.3,2 \mathrm{H}), 1.30-1.27$ $(\mathrm{m}, 2 \mathrm{H}), 0.51(\mathrm{t}, J=7.3 \mathrm{~Hz}, 3 \mathrm{H}) ;{ }^{13} \mathrm{C}$ NMR $\left(125 \mathrm{MHz}, \mathrm{CDCl}_{3}\right) \delta 136.7$, 135.4, 134.3, 133.8, 132.3, 131.5, 129.3, 128.8, 128.7, 128.3, 128.0, 127.8, 127.3, 125.3, 123.1, 109.7, 46.8, 24.4, 11.1; All additional spectral information are similar to those reported by Namy and Farcas. ${ }^{23}$

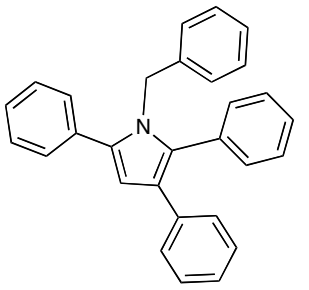

1-benzyl-2,3,5-triphenyl-1-H-pyrrole (45): Purified with 2\% ethyl acetate/hexanes yielding $78 \mathrm{mg}(65 \%)$ of $\mathbf{4 5}$ white solid. $\mathrm{R}_{f}=0.57$ (25\% ethyl acetate/hexanes); mp: $162-165^{\circ} \mathrm{C}$ (lit. $165-167^{\circ} \mathrm{C}$ ). ${ }^{1} \mathrm{H}$ NMR $(500 \mathrm{MHz}$, $\left.\mathrm{CDCl}_{3}\right)$ d 7.45-7.12 (m, 18H), 6.69-6.68 (m, 2H), $6.61(\mathrm{~s}, 1 \mathrm{H}), 5.13(\mathrm{~s}, 2 \mathrm{H})$; ${ }^{13} \mathrm{C}$ NMR $\left(125 \mathrm{MHz}, \mathrm{CDCl}_{3}\right) \delta 139.4,136.4,135.8,133.7,133.4,132.6$, 131.6, 129.3, 128.7, 128.6, 128.5, 128.3, 127.9, 127.8, 127.4, 126.3, 125.4, 123.5, 109.7, 48.7; All additional spectral information are similar to those reported by Yoshida and coworkers. ${ }^{24}$

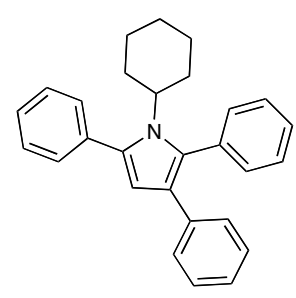

1-cyclohexyl-2,3,5-triphenyl-1H-pyrrole (46): Purified with 2\% ether/hexanes, yielding $66 \mathrm{mg}(64 \%)$ of $\mathbf{4 6}$ as a white solid. $\mathrm{R}_{f}=0.75(25 \%$ ethyl acetate/hexanes); mp: $165-167^{\circ} \mathrm{C} .{ }^{1} \mathrm{H}$ NMR $\left(500 \mathrm{MHz}, \mathrm{CDCl}_{3}\right) \delta 7.51-$ $7.39(\mathrm{~m}, 9 \mathrm{H}), 7.13-7.03(\mathrm{~m}, 6 \mathrm{H}), 6.39(\mathrm{~s}, 1 \mathrm{H}), 3.94(\mathrm{~m}, 1 \mathrm{H}), 1.86(\mathrm{~d}, J=11.2$ $\mathrm{Hz}, 2 \mathrm{H}), 1.61-0.72(\mathrm{~m}, 8 \mathrm{H}) ;{ }^{13} \mathrm{C}$ NMR $(125 \mathrm{MHz}) \delta 136.7,135.2,134.8$, 132.6, 131.7, 130.6, 128.4, 128.2, 128.1, 127.7, 127.5, 125.0, 123.0, 109.9, 58.9, 34.4, 26.7, 25.5; MALDI: Mass (m/e) calculated for $\mathrm{C}_{28} \mathrm{H}_{27} \mathrm{~N}$ : 377.5 .

Found 377.1. 


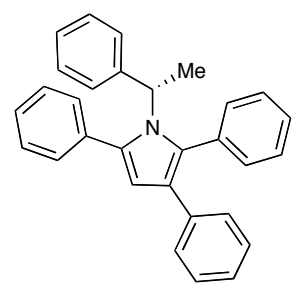

2,3,5-triphenyl-1-((S)-1-phenylethyl)-1- $\boldsymbol{H}$-pyrrole (47): Purified with 5\% ether/hexanes, yielding $60 \mathrm{mg}(55 \%)$ of $\mathbf{4 7}$ as yellow oil. $\mathrm{R}_{f}=0.71(25 \%$ ethyl acetate/hexanes); ${ }^{1} \mathrm{H}$ NMR $\left(500 \mathrm{MHz}, \mathrm{CDCl}_{3}\right) \delta$ 7.29-7.06 $(\mathrm{m}, 18 \mathrm{H})$, 7.05-6.91 (m, 2H), $6.50(\mathrm{~s}, 1 \mathrm{H}), 5.52(\mathrm{q}, J=5.9 \mathrm{~Hz}, 1 \mathrm{H}), 1.58(\mathrm{~d}, J=7.2 \mathrm{~Hz}$, $3 \mathrm{H}) ;{ }^{13} \mathrm{C}$ NMR $(125 \mathrm{MHz}) \delta 143.4,136.5,135.6,134.5,134.2,132.5,131.8$, $130.5,128.4,128.3,128.2,127.9,127.6,127.5,127.0,126.5,125.2,123.2$, 109.8, 54.0, 20.0; MALDI: Mass (m/e) calculated for $\mathrm{C}_{30} \mathrm{H}_{25} \mathrm{~N}$ : 399.5. Found

399.2 .

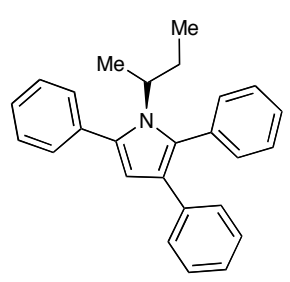

(R)-1-sec-butyl-2,3,5-triphenyl-1H-pyrrole (48): Purified with 2\% ether/hexanes, yielding $54 \mathrm{mg}(56 \%)$ of $\mathbf{4 8}$ as a yellow oil. $\mathbf{R}_{f}=0.70(25 \%$ ethyl acetate/hexanes); ${ }^{1} \mathrm{H}$ NMR $\left(500 \mathrm{MHz}, \mathrm{CDCl}_{3}\right) \delta$ 7.50-7.36 $(\mathrm{m}, 10 \mathrm{H})$, 7.12-7.03(m, 5H), $6.38(\mathrm{~s}, 1 \mathrm{H}), 4.10(\mathrm{~m}, 1 \mathrm{H}), 1.55(\mathrm{~m}, 1 \mathrm{H}), 1.37-1.35(\mathrm{~m}, 1 \mathrm{H})$, $1.32(\mathrm{~d}, J=7.1 \mathrm{~Hz}, 3 \mathrm{H}), 0.62(\mathrm{t}, J=7.3 \mathrm{~Hz}, 3 \mathrm{H}) ;{ }^{13} \mathrm{C}$ NMR $(125 \mathrm{MHz}) \delta$ $136.7,135.3,134.8,132.5,130.6,128.5,128.2,128.1,128.0,127.7,127.5$, 125.0, 109.9, 55.6, 30.2, 22.8, 11.3; MALDI: Mass (m/e) calculated for $\mathrm{C}_{26} \mathrm{H}_{25} \mathrm{~N}$ : 351.4 Found 351.4 .

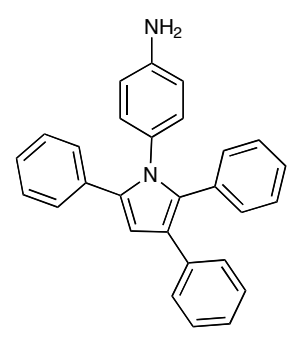

4-(2,3,5-triphenyl-1H-pyrrol-1-yl)benzeneamine (49): Purified with $20 \%$ ethyl acetate/hexanes to give $37 \mathrm{mg}(70 \%)$ of $\mathbf{4 9}$ as an oil. $\mathrm{R}_{f}=0.41(25 \%$ ethyl acetate/hexanes); IR (film) 3468, 3378, 3054, 2916, 2848, 1621, 1600, $1515 \mathrm{~cm}^{-1} ;{ }^{1} \mathrm{H}$ NMR $\left(500 \mathrm{MHz}, \mathrm{CDCl}_{3}\right) \delta$ 7.37-7.13 (m, $\left.15 \mathrm{H}\right), 6.78(\mathrm{~m}, 2 \mathrm{H})$, $6.69(\mathrm{~s}, 1 \mathrm{H}), 6.48(\mathrm{~m}, 2 \mathrm{H}) ;{ }^{13} \mathrm{C}$ NMR $(125 \mathrm{MHz}) \delta 145.2,136.6,135.1$, $133.3,131.8,130.1,128.8,128.6,128.4,128.3,128.0,127.0,126.4,125.6$, 123.3, 116.6, 115.1, 109.7; MALDI: Mass (m/e) calculated for $\mathrm{C}_{29} \mathrm{H}_{22} \mathrm{~N}_{2}$ :

386.5. Found 386.6.

\section{Procedure for Microwave Reaction (compound 36):}

A conical test tube was charged with the thiazolium salt ( $14 \mathrm{mg}, 0.055 \mathrm{mmol}, 0.20$ equiv). To the conical tube, benzoyltrimethylsilane ( $100 \mathrm{mg}, 0.55 \mathrm{mmol}, 2.0$ equiv) in THF $(0.25 \mathrm{~mL})$ was added by syringe to the test tube followed by the addition of DBU $(13 \mathrm{~mL}, 0.083 \mathrm{mmol}, 0.30$ equiv). To reaction mixture chalcone $(61 \mathrm{mg}, 0.275 \mathrm{mmol}, 1.0$ equiv) in THF $(0.25 \mathrm{~mL})$ was added by syringe followed by the addition of 2-propanol ( $83 \mathrm{~mL}, 1.10 \mathrm{mmol}, 4.0$ equiv). The conical tube was capped with septa and crimped aluminum collar and heated for 15 minutes in the microwave at $160^{\circ} \mathrm{C}$. After this first heating cycle, the septa was removed and a solution of amine (77 mg, $0.825 \mathrm{mmol}, 3.0$ equiv) followed by $p$-toluenesulfonic acid $(104 \mathrm{mg}, 0.55 \mathrm{mmol}$, 2.0 equiv) in ethanol $(0.5 \mathrm{~mL})$ and $4 \AA$ molecular sieves (spatula tip) were added to the reaction mixture and heated in the microwave for an additional 15 minutes at $160{ }^{\circ} \mathrm{C}$. Upon cooling to room temperature, the reaction mixture was diluted with ethyl acetate $(30 \mathrm{~mL})$ and washed with water $(10 \mathrm{~mL})$. The aqueous layer was washed with ethyl acetate $(3 \times 30 \mathrm{~mL})$ and the combined the organic extracts were washed with brine $(10 \mathrm{~mL})$ and dried over sodium sulfate, filtered, and concentrated in vacuo. The resulting residue was purified by flash column chromatography on silica gel (2\% ethyl acetate/hexanes) to give $58 \mathrm{mg} \mathrm{(55 \% )} \mathrm{of} \mathbf{3 6}$ as a white solid. 
General Procedure for Thiazolium-Catalyzed $\alpha$-Aminoketone Synthesis:

A screw-capped tube was charged with the thiazolium salt $(20 \mathrm{mg}, 0.08 \mathrm{mmol})$ in a nitrogenfilled dry box. The tube was removed from the box and placed under a positive pressure of nitrogen. Benzoyltrimethylsilane $(84 \mathrm{mg}, 0.47 \mathrm{mmol})$ in $\mathrm{CHCl}_{3}(0.25 \mathrm{~mL})$ was added by syringe followed by the addition of DBU $(12 \mathrm{~mL}, 0.08 \mathrm{mmol})$. The reaction mixture was heated to 60 ${ }^{\circ} \mathrm{C}$ after which the phosphoryl imine $(0.26 \mathrm{mmol})$ in $\mathrm{CHCl}_{3}(0.25 \mathrm{~mL})$ was added by syringe followed by the addition of isopropanol $(80 \mathrm{~mL}, 1.05 \mathrm{mmol})$. The reaction was allowed to stir at $60{ }^{\circ} \mathrm{C}$ for 24 hours. Upon completion by HPLC the reaction was cooled to room temperature, diluted with methylene chloride $(20 \mathrm{~mL})$ and washed with water $(20 \mathrm{~mL})$. The aqueous layer was washed with methylene chloride $(3 \times 30 \mathrm{~mL})$ and the combined organic extracts were dried over sodium sulfate, filtered, and concentrated in vacuo. The resulting residue was purified by flash column chromatography on silica gel.

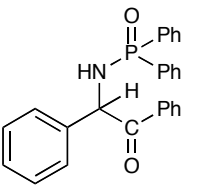

2-(Diphenylphosphinamide)-1,2-diphenylethanone (51): Purified with 1\% methanol/ethyl acetate, yielding $100 \mathrm{mg}(93 \%)$ of $\mathbf{5 1}$ as a white foam. $\mathbf{R}_{f}=0.69$ (100\% ethyl acetate); Mp: $135.5-136{ }^{\circ} \mathrm{C}$; IR (film) 3184, 3057, 1682, $1200 \mathrm{~cm}^{-1}$; ${ }^{1} \mathrm{H}$ NMR $\left(500 \mathrm{MHz}, \mathrm{CDCl}_{3}\right)$ d 7.86-7.81 (m, 4H); 7.69-7.65 (m, 2H); 7.47-7.39 (m, $4 \mathrm{H})$; 7.33-7.30 (M, 4H); $7.15(\mathrm{bs}, 5 \mathrm{H}) ; 5.96(\mathrm{dd}, J=8.9,8.9 \mathrm{~Hz}, 1 \mathrm{H}) ; 4.96(\mathrm{dd}, J=7.9,7.9 \mathrm{~Hz}$, $1 \mathrm{H}) ;{ }^{13} \mathrm{C}$ NMR (500 MHz, CDCl) d 196.7, 138.9, 134.4, 133.8, 133.5, 132.7, 132.7, 132.5, 132.2, 131.9, 131.8, 131.7, 129.4, 129.2, 128.9, 128.8, 128.8, 128.4, 128.3, 128.2, 128.2, 59.4; LRMS (MALDI-TOF): Mass calculated for $\mathrm{C}_{26} \mathrm{H}_{22} \mathrm{NO}_{2} \mathrm{P}[\mathrm{M}+\mathrm{H}]^{+}, 412.4$. Found 412.2.

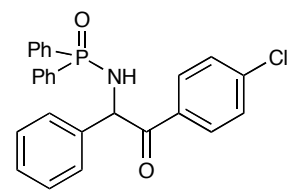

1-(4-Chlorophenyl)-2-(diphenylphosphinamide)-2-phenylethanone (52): Purified with $1 \%$ methanol/ethyl acetate, yielding $105 \mathrm{mg}(90 \%)$ of $\mathbf{5 2}$ as a white foam. $\mathrm{R}_{f}=0.74$ (100\% ethyl acetate); $\mathrm{Mp}: 150-151^{\circ} \mathrm{C}$; IR (film) 3170 , 3057, 1685, $1200 \mathrm{~cm}^{-1}$; ${ }^{1} \mathrm{H}$ NMR $\left(500 \mathrm{MHz} \mathrm{CDCl}_{3}\right)$ d 7.84-7.82 (m, 3H); 7.80-7.65 (m, 2H); 7.48-7.40 (m, 3H); 7.39-7.29 (m, 3H); 7.16-7.14 (m, 4H); $5.91(\mathrm{dd}, J=8.6,8.6 \mathrm{~Hz}, 1 \mathrm{H}) ; 4.91(\mathrm{dd}, J=8.1,8.1 \mathrm{~Hz}, 1 \mathrm{H}) ;{ }^{13} \mathrm{C} \mathrm{NMR}\left(500 \mathrm{MHz}, \mathrm{CDCl}_{3}\right) \mathrm{d}$ 195.6, 140.3, 138.7, 138.5, 132.8, 132.7, 132.7, 132.5, 132.3, 131.9, 131.8, 131.6, 130.7, 129.3, 129.2, 128.9, 128.8, 128.5, 128.4, 128.3, 128.2, 59.4; LRMS (MALDI-TOF): Mass calculated for $\mathrm{C}_{26} \mathrm{H}_{21} \mathrm{ClNO}_{2} \mathrm{P}[\mathrm{M}+\mathrm{H}]^{+}$, 446.1. Found 447.4.

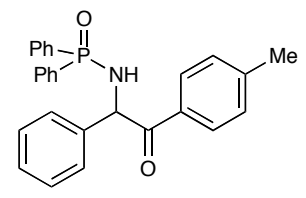

2-(Diphenylphosphinamide)-2-phenyl-1-p-tolylethanone (53): Purified with $1 \%$ methanol/ethyl acetate, yielding $90 \mathrm{mg}(81 \%)$ of $\mathbf{5 3}$ as a white foam. $\mathrm{R}_{f}=0.74$ (100\% ethyl acetate); Mp: $138-138.5^{\circ} \mathrm{C}$; IR (film) 3177,3057 , $1680,1202 \mathrm{~cm}^{-1} ;{ }^{1} \mathrm{H} \mathrm{NMR}\left(500 \mathrm{MHz}, \mathrm{CDCl}_{3}\right) \mathrm{d}$ 7.85-7.81 (m, 2H); 7.77-7.75 $(\mathrm{m}, 2 \mathrm{H}) ; 7.69-7.65(\mathrm{~m}, 2 \mathrm{H}), 7.48-7.28(\mathrm{~m}, 4 \mathrm{H}) ; 7.15-7.11(\mathrm{~m}, 6 \mathrm{H}) ; 5.94(\mathrm{dd}$, $J=10.1,8.2 \mathrm{~Hz}, 1 \mathrm{H}) ; 4.99(\mathrm{dd}, J=8.2,8.2 \mathrm{~Hz}, 1 \mathrm{H}) ; 2.31(\mathrm{~s}, 3 \mathrm{H}) ;{ }^{13} \mathrm{C} \mathrm{NMR}\left(500 \mathrm{MHz}, \mathrm{CDCl}_{3}\right)$ d 196.2, 144.9, 139.3, 132..7, 132.7, 132.2, 131.9, 131.8, 129.5, 129.1, 128.9, 128.8, 128.4, 128.3, 128.2, 128.1, 59.2, 21.9; LRMS (MALDI-TOF): Mass calculated for $\mathrm{C}_{27} \mathrm{H}_{24} \mathrm{NO}_{2} \mathrm{P}$ $[\mathrm{M}+\mathrm{H}]^{+}, 426.2$. Found 427.8.

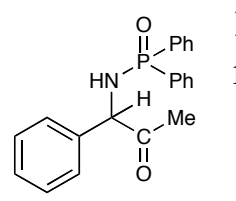

1-(Diphenylphosphinamide)-1-phenylpropan-2-one (54): Purified with $1 \%$ methanol/ethyl acetate, yielding $80 \mathrm{mg}(87 \%)$ of $\mathbf{5 4}$ as a white foam. $\mathbf{R}_{f}=0.46$ 
(100\% ethyl acetate); Mp: 99-100 ${ }^{\circ} \mathrm{C}$; IR (film) $3168,3057,1717,1196 \mathrm{~cm}^{-1} ;{ }^{1} \mathrm{H}$ NMR (500 $\left.\mathrm{MHz}, \mathrm{CDCl}_{3}\right)$ d 7.84-7.80 (m, 2H); 7.61-7.57 (m, 2H); 7.49-7.41 (m, 3H); 7.34-7.32 (m, 1H); 7.21-7.17 (m, 4H); 7.07-7.06 (m, 2H); $5.05(\mathrm{dd}, J=11.1,6.7 \mathrm{~Hz}, 1 \mathrm{H}) ; 4.84(\mathrm{dd}, J=6.2,6.2 \mathrm{~Hz}$, 1H); 2.02 (s, 3H) ${ }_{13}^{13} \mathrm{C}$ NMR (500 MHz, CDCl) d 204.0, 138.3, 132.7, 132.7, 132.4, 132.3, 131.8, 131.8, 129.1, 128.9, 128.8, 128.4, 128.3, 128.2, 128.1, 64.0, 27.1; LRMS (MALDI-TOF): Mass calculated for $\mathrm{C}_{21} \mathrm{H}_{20} \mathrm{NO}_{2} \mathrm{P}[\mathrm{M}+\mathrm{H}+\mathrm{Na}]^{+}$, 372.1. Found 372.4.

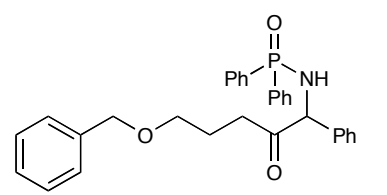

5-(Benzyloxy)-1-(diphenylphosphinamide)-1-phenylpentan-2-one (55): Purified with $1 \%$ methanol/ethyl acetate, yielding $40 \mathrm{mg}(63 \%)$ of $\mathbf{5 5}$ as an orange oil. $\mathrm{R}_{f}=0.70$ (100\% ethyl acetate); IR (film) 3175,3059 , $2928,1718,1198 \mathrm{~cm}^{-1} ;{ }^{1} \mathrm{H}$ NMR $\left(500 \mathrm{MHz}, \mathrm{CDCl}_{3}\right) \mathrm{d}$ 7.83-7.79 (m, 2H); 7.62-7.58 (m, 2H); 7.50-7.21 (m, 16H); $7.06(\mathrm{~m}, 2 \mathrm{H}) ; 5.03(\mathrm{dd}, J=10.4,7.0 \mathrm{~Hz}, 1 \mathrm{H}) ; 4.84(\mathrm{~m}$, $1 \mathrm{H}) ; 4.33(\mathrm{q}, J=12.2 \mathrm{~Hz}, 2 \mathrm{H}) ; 3.30(\mathrm{~m}, 2 \mathrm{H}) ; 2.43(\mathrm{~m}, 2 \mathrm{H}) ; 1.78(\mathrm{~m}, 2 \mathrm{H}) ;{ }^{13} \mathrm{C}$ NMR $(500 \mathrm{MHz}$, $\mathrm{CDCl}_{3}$ ) d 210.7, 138.4, 132.7, 132.6, 132.3, 131.9, 131.8, 129.1, 128.9, 128.8, 128.6, 128.4, 128.3, 128.3, 128.2, 127.8, 127.8, 69.0, 63.5, 36.4, 24.1; LRMS (MALDI-TOF): Mass calculated for $\mathrm{C}_{30} \mathrm{H}_{30} \mathrm{NO}_{3} \mathrm{P}[\mathrm{M}+\mathrm{H}+\mathrm{Na}]^{+}$, 507.2. Found 507.2.

2-(4-Chlorophenyl)-2-(diphenylphosphinamide)-1-phenylethanone (56):

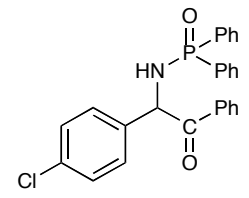

Purified with $1 \%$ methanol/ethyl acetate, yielding $100 \mathrm{mg}(85 \%)$ of $\mathbf{5 6}$ as a white foam. $\mathrm{R}_{f}=0.69$ (100\% ethyl acetate); Mp: $152-152.5^{\circ} \mathrm{C}$; IR (film) 3171 , $3057,1685,1197 \mathrm{~cm}^{-1} ;{ }^{1} \mathrm{H}$ NMR $\left(500 \mathrm{MHz}, \mathrm{CDCl}_{3}\right) \mathrm{d}$ 7.82-7.81 (m, 4H); 7.67$7.63(\mathrm{~m}, 2 \mathrm{H}) ; 7.48-7.28(\mathrm{~m}, 8 \mathrm{H}) ; 7.11-7.05(\mathrm{M}, 4 \mathrm{H}) ; 5.94(\mathrm{dd}, J=8.5,8.5 \mathrm{~Hz}$, $1 \mathrm{H}) ; 4.96(\mathrm{dd}, J=7.9,7.9 \mathrm{~Hz}, 1 \mathrm{H}) ;{ }^{13} \mathrm{C}$ NMR $\left(500 \mathrm{MHz}, \mathrm{CDCl}_{3}\right)$ d 196.3, 137.6, 134.2, 134.1, 134.1, 132.7, 132.6, 132.3, 131.9, 131.9, 131.8, 129.6, 129.3, 128.9, 128.8, 128.5, 128.4, 58.7; LRMS (MALDI-TOF): Mass calculated for $\mathrm{C}_{26} \mathrm{H}_{21} \mathrm{ClNO}_{2} \mathrm{P}[\mathrm{M}+\mathrm{H}+\mathrm{Na}]^{+}$, 469.1. Found 469.3.

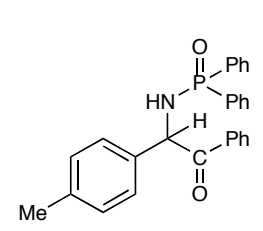

2-(Diphenylphosphinamide)-1-phenyl-2-p-tolylethanone (57): Purified with $1 \%$ methanol/ethyl acetate, yielding $104 \mathrm{mg}(94 \%)$ of $\mathbf{5 7}$ as a white foam. $\mathbf{R}_{f}=$ 0.69 (100\% ethyl acetate); Mp: $151-152^{\circ} \mathrm{C}$; IR (film) $3179,3056,2976,2918$, $1684,1437,1199 \mathrm{~cm}^{-1} ;{ }^{1} \mathrm{H}$ NMR $\left(500 \mathrm{MHz}, \mathrm{CDCl}_{3}\right) \mathrm{d}$ 7.86-7.82 (m, 4H); 7.727.68 (m, 2H); 7.48-7.41 (m, 4H); 7.33-7.27 (m, 4H) 7.04-7.03 (m, 2H); 6.98$6.96(\mathrm{~m}, 2 \mathrm{H}) ; 5.92(\mathrm{dd}, J=9.2,9.2 \mathrm{~Hz}, 1 \mathrm{H}) ; 4.91(\mathrm{dd}, J=8.2,8.2 \mathrm{~Hz}, 1 \mathrm{H}) ; 2.23(\mathrm{~s}$, $3 \mathrm{H}) ;{ }^{13} \mathrm{C}$ NMR (500 MHz, CDCl) d 196.8, 138.0, 136.0, 134.5, 133.8, 133.5, 132.8, 132.7, 132.5, 132.2, 132.0, 131.9, 131.8, 129.9, 129.3, 128.9, 128.8, 128.4, 128.3, 128.1, 59.2, 21.3; MALDI: Mass (m/e) calculated for $\mathrm{C}_{27} \mathrm{H}_{24} \mathrm{NO}_{2} \mathrm{P}[\mathrm{M}+\mathrm{H}+\mathrm{Na}]^{+}$, 449.2: Found 449.4.

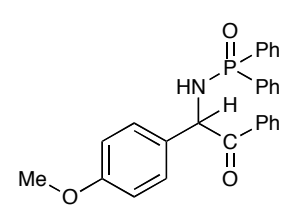

2-(Diphenylphosphinamide)-2-(4-methoxyphenyl)-1-phenylethanone (58):

Purified with $1 \%$ methanol/ethyl acetate, yielding $100 \mathrm{mg}(86 \%)$ of $\mathbf{5 8}$ as a white foam. $\mathrm{R}_{f}=0.58$ ( $100 \%$ ethyl acetate); Mp: $157-158^{\circ} \mathrm{C}$; IR (film) 3179, 3055, 2957, 1684, $1198 \mathrm{~cm}^{-1} ;{ }^{1} \mathrm{H}$ NMR $\left(500 \mathrm{MHz}, \mathrm{CDCl}_{3}\right)$ d 7.85-7.81 (m, 4H); 7.71-7.67 (m, 2H); 7.47-7.41 (m, 4H); 7.33-7.29 (m, 4H); 7.06-7.05 (m, 2H); 6.69-6.67 (m, 2H); $5.92(\mathrm{dd}, J=8.9,8.9 \mathrm{~Hz}, 1 \mathrm{H}) ; 4.91(\mathrm{dd}, J=7.8,7.8 \mathrm{~Hz}, 1 \mathrm{H}) ; 3.69(\mathrm{~s}$, $3 \mathrm{H}) ;{ }^{13} \mathrm{C}$ NMR $(500 \mathrm{MHz}, \mathrm{CDCl}) \mathrm{d} 196.7,159.5,134.5,133.7,132.8,132.7,132.2,131.9$, 131.9, 131.1, 129.5, 129.3, 128.9, 128.8, 128.4, 128.3, 114.6, 58.8, 55.4; LRMS (MALDI-TOF): Mass calculated for $\mathrm{C}_{27} \mathrm{H}_{24} \mathrm{NO}_{3} \mathrm{P}[\mathrm{M}+\mathrm{H}+\mathrm{Na}]^{+}$, 465.2. Found 465.8. 


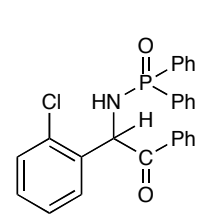

2-(2-Chlorophenyl)-2-(diphenylphosphinamide)-1-phenylethanone (59):

Purified with $1 \%$ methanol/ethyl acetate, yielding $90 \mathrm{mg}(77 \%)$ of $\mathbf{5 9}$ as a white foam. $\mathrm{R}_{f}=0.69$ ( $100 \%$ ethyl acetate); Mp: $158-158.5^{\circ} \mathrm{C}$; IR (film) 3149,3058 , $1685,1197 \mathrm{~cm}^{-1} ;{ }^{1} \mathrm{H}$ NMR $\left(500 \mathrm{MHz}, \mathrm{CDCl}_{3}\right) \mathrm{d}$ 7.90-7.83 (m, 4H); 7.70-7.67 (m, $2 \mathrm{H}) ; 7.47-7.38$ (m, 7H); 7.37-7.31 (m, 3H); 7.25-7.06 (m, 3H); 6.35 (dd, $J=10.2$, $7.5 \mathrm{~Hz}, 1 \mathrm{H}) ; 5.09(\mathrm{dd}, J=7.2,7.2 \mathrm{~Hz}, 1 \mathrm{H}) ;{ }^{13} \mathrm{C} \mathrm{NMR}\left(500 \mathrm{MHz}, \mathrm{CDCl}_{3}\right)$ d 196.3, 136.9, 134.2, 134.0, 133.6, 132.9, 132.8, 132.5, 132.2, 131.8, 131.7, 131.5, 130.3, 129.8, 129.6, 129.1, 128.9, 128.8, 128.3, 128.2, 127.7, 56.4; LRMS (MALDI-TOF): Mass calculated for $\mathrm{C}_{26} \mathrm{H}_{21} \mathrm{ClNO}_{2} \mathrm{P}$ $[\mathrm{M}+\mathrm{H}+\mathrm{Na}]^{+}, 469.1$. Found 469.6.

2-(Diphenylphosphinamide)-1-phenyl-2-(thiophen-2-yl)ethanone (60): Purified with $1 \%$ methanol/ethyl acetate, yielding $87 \mathrm{mg} \mathrm{(80 \% )} \mathrm{of} \mathbf{6 0}$ as a white foam. $\mathrm{R}_{f}=0.61$ (100\% ethyl acetate); IR (film) $3187,2058,1685,1199 \mathrm{~cm}^{-1} ;{ }^{1} \mathrm{H}$ NMR (500 MHz, $\left.\mathrm{CDCl}_{3}\right)$ d 7.92-7.80 (m, 5H); 7.51-7.36 (m, 8H); 7.16-7.15 (m, $1 \mathrm{H}) ; 6.77(\mathrm{~s}, 2 \mathrm{H}) ; 6.24(\mathrm{dd}, J=9.3,9.3 \mathrm{~Hz}, 1 \mathrm{H}) ; 4.89(\mathrm{dd}, J=8.4,8.4 \mathrm{~Hz}$, $1 \mathrm{H}) ;{ }^{13} \mathrm{C}$ NMR (500 MHz, CDCl $)$ d 195.6, 141.9, 134.1, 133.2, 132.7, 132.6, 132.4, 132.1, 132.0, 131.9, 131.6, 129.4, 128.9, 128.8, 128.6, 128.5, 127.3, 127..2, 126.5, 54.1; LRMS (MALDI-TOF): Mass calculated for $\mathrm{C}_{24} \mathrm{H}_{20} \mathrm{NO}_{2} \mathrm{PS}[\mathrm{M}+\mathrm{H}+\mathrm{Na}]^{+}$, 441.1. Found 440.7.

Determination of Silyl Acceptor in Addition to Chalcone: An authentic sample of 3trimethylsilyloxy-octane was prepared by combining 3-octanol $(1 \mathrm{~mL}, 6.29 \mathrm{mmol})$, trimethylsilyl chloride $(2.4 \mathrm{~mL}, 18.9 \mathrm{mmol})$, and triethylamine $(2.6 \mathrm{~mL}, 18.9 \mathrm{mmol})$ in $\mathrm{CH}_{2} \mathrm{Cl}_{2}(16 \mathrm{~mL})$ and stirring at room temperature for 24 hours. Upon completion, the reaction mixture was diluted with pentane, washed with water $(30 \mathrm{~mL})$ and saturated $\mathrm{CuSO}_{4}(3 \times 30 \mathrm{~mL})$. The organic layer was dried over $\mathrm{Na}_{2} \mathrm{SO}_{4}$, filtered, and concentrated in vacuo. A retention time (3.95 min) was collected on an Agilent Technologies 6890N gas chromatograph (equipped with Agilent 19091J$413 \mathrm{HP}-55 \%$ phenylmethylsiloxane capillary column- $30.0 \mathrm{~m}$ x $320 \mathrm{~mm}$ x $0.25 \mathrm{~mm}$ nominal) using the following conditions: $70{ }^{\circ} \mathrm{C}$ for $1 \mathrm{~min}$ then a ramp of $25^{\circ} \mathrm{C} / \mathrm{min}$ to $285^{\circ} \mathrm{C}$ with 3 minute hold.

For the experiment, a screw-capped test tube was charged with 3-benzyl-4,5-dimethylthiazolium bromide $(51 \mathrm{mg}, 0.183 \mathrm{mmol})$ in a nitrogen filled glove box. The test tube was removed from box and put under a positive pressure of nitrogen. Benzoyltrimethylsilane $(50 \mathrm{mg}, 0.274 \mathrm{mmol})$ in THF $(0.25 \mathrm{~mL})$ was added by syringe to the test tube followed by the addition of DBU (27 $\mathrm{mL}, 0.183 \mathrm{mmol})$. The reaction mixture was heated to $70^{\circ} \mathrm{C}$ after which the chalcone $(0.183$ $\mathrm{mmol})$ in THF $(0.25 \mathrm{~mL})$ was added by syringe followed by the addition of 3-octanol $(50 \mathrm{~mL}$, $0.314 \mathrm{mmol})$. The reaction was allowed to stir at $70^{\circ} \mathrm{C}$ for 4 hours. At this time an aliquot was filtered through glass wool and analyzed by GC (see above for details). The following retention times were observed: $3.25 \mathrm{~min}$ (3-octanol), $3.95 \mathrm{~min}$ (3-trimethylsilyloxy-octane), $4.99 \mathrm{~min}$ (benzoyltrimethylsilane), $8.37 \mathrm{~min}$ (chalcone), $10.89 \mathrm{~min}$ (1,2,4-triphenylbutane-1,4-dione).

Determination of Silyl Acceptor in Additions to N-Phosphinoylimines: An authentic sample of 3-trimethylsilyloxy-octane was prepared by combining 3-octanol $(1 \mathrm{~mL}, 6.29 \mathrm{mmol})$, 
trimethylsilyl chloride $(2.4 \mathrm{~mL}, 18.9 \mathrm{mmol})$, and triethylamine $(2.6 \mathrm{~mL}, 18.9 \mathrm{mmol})$ in $\mathrm{CH}_{2} \mathrm{Cl}_{2}(16 \mathrm{~mL})$ and stirring at room temperature for 24 hours. Upon completion, the reaction mixture was diluted with pentane, washed with water $(30 \mathrm{~mL})$ and saturated aqueous $\mathrm{CuSO}_{4}(3 \mathrm{x}$ $30 \mathrm{~mL}$ ). The organic layer was dried over $\mathrm{Na}_{2} \mathrm{SO}_{4}$, filtered, and concentrated in vacuo. A retention time (4.720 min) was collected on an Agilent Technologies $6890 \mathrm{~N}$ gas chromatograph (equipped with Agilent 19091J-413 HP-5 5\% phenylmethylsiloxane capillary column- $30.0 \mathrm{~m} \mathrm{x}$ $320 \mathrm{~mm} \times 0.25 \mathrm{~mm}$ nominal) using the following conditions: $70^{\circ} \mathrm{C}$ for $1 \mathrm{~min}$ then a ramp of 25 ${ }^{\circ} \mathrm{C} /$ min to $285^{\circ} \mathrm{C}$ with 3 minute hold.

In the experiment, a screw-capped tube was charged with 3,4,5-trimethylthiazolium iodide (10, $10 \mathrm{mg}, 0.04 \mathrm{mmol}$ ) in a nitrogen filled glove box. The tube was removed from box and put under a positive pressure of nitrogen. Benzoyltrimethylsilane $(41 \mathrm{mg}, 0.23 \mathrm{mmol})$ in $\mathrm{CHCl}_{3}$ $(0.25 \mathrm{~mL})$ was added by syringe to the tube followed by the addition of DBU (12 mL, 0.04 mmol). The reaction mixture was heated to $60^{\circ} \mathrm{C}$ after which imine $12(0.13 \mathrm{mmol})$ in $\mathrm{CHCl}_{3}$ $(0.25 \mathrm{~mL})$ was added by syringe followed by the addition of 3 -octanol $(83 \mathrm{~mL}, 0.52 \mathrm{mmol})$. The reaction was allowed to stir at $60{ }^{\circ} \mathrm{C}$ for 4 hours. At this time an aliquot was filtered through glass wool and analyzed by GC (see above for details). The following retention times were observed: $3.89 \mathrm{~min}$ (3-octanol), $4.71 \mathrm{~min}$ (3-trimethylsilyloxy-octane), $5.96 \mathrm{~min}$ (benzoyltrimethylsilane), $15.00 \mathrm{~min}$ (imine). The a-amino ketone product (13) was not observed due to its molecular weight and highly polar nature.

\section{General Procedure for the Preparation of Protected Thiazolium Carbinols:}

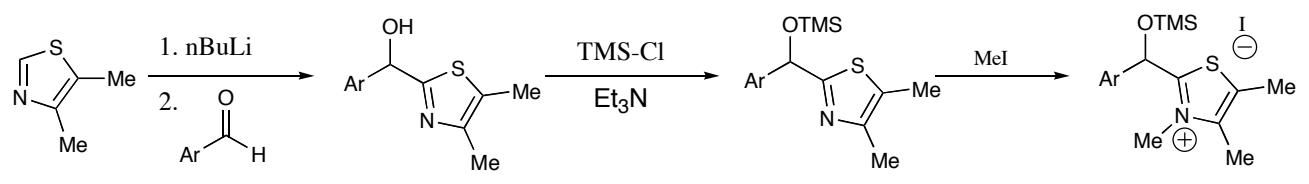

\section{Synthesis of Carbinol:}

A flame-dried round bottom flask under $\mathrm{N}_{2}$ was charged with 4,5-dimethylthiazole $(1 \mathrm{~mL}, 9.45$ mmol). THF ( $35 \mathrm{~mL})$ was added and the solution was cooled to $-78^{\circ} \mathrm{C}$. $n$-Butyl lithium $(2.03$ $\mathrm{M}, 5.6 \mathrm{~mL}, 11.3 \mathrm{mmol}$ ) was added dropwise to the cold reaction mixture. The resulting dark red solution was stirred for $45 \mathrm{~min}$ at $-78^{\circ} \mathrm{C}$. Benzaldehyde $(28.4 \mathrm{mmol})$ was then added, causing the solution to turn yellow, and the reaction was stirred for $1 \mathrm{~h}$ at $-78{ }^{\circ} \mathrm{C}$. The reaction was warmed to room temperature and stirred for $2 \mathrm{~h}$. At this time, water $(50 \mathrm{~mL})$ was added to quench the reaction. The reaction mixture was diluted with ethyl acetate $(200 \mathrm{~mL})$ and washed with water $(200 \mathrm{~mL})$. The aqueous layer was extracted two additional times with ethyl acetate $(100 \mathrm{~mL})$. The combined organic extracts were dried over sodium sulfate, filtered and concentrated in vacuo. The resulting residue was purified by recrystallization (ethyl acetate/hexanes).

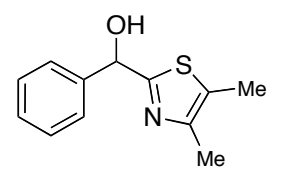

(4,5-Dimethylthiazol-2-yl)(phenyl)methanol: Purified by recrystallization (ethyl acetate/hexanes), yielding $1.80 \mathrm{~g}(87 \%)$ as a white solid. ${ }^{1} \mathrm{H}$ NMR (500 $\mathrm{MHz}, \mathrm{CDCl}_{3}$ ) $\delta 7.46$ (m, 2H); 7.38 (m, 3H); 5.95 (s, 3H); 2.30 (s, 3H); 2.29 (s, $3 \mathrm{H})$. 
Protection and Alkylation of Carbinol:

To a flame-dried round bottom flask under $\mathrm{N}_{2}$ was added the carbinol $(8.21 \mathrm{mmol})$ followed by dichloromethane $(36 \mathrm{~mL})$. Next, trimethylsilylchloride $(3.12 \mathrm{~mL}, 24.6 \mathrm{mmol})$ was added followed by triethylamine $(1.26 \mathrm{~mL}, 9.03 \mathrm{mmol})$. The resulting solution was then stirred overnight at room temperature. The reaction mixture was diluted with dichloromethane (100 $\mathrm{mL})$ and washed three times with brine $(200 \mathrm{~mL})$. The combined aqueous layers were washed with dichloromethane. The combined organic layers were dried over $\mathrm{Na}_{2} \mathrm{SO}_{4}$, filtered and concentrated in vacuo. The pale yellow oil (2.08 g, 87\% yield) was used in the next step without further purification.

1

To the protected carbinol in a flame-dried round bottom flask was added iodomethane (10 eq) and the reaction was heated at reflux at $70{ }^{\circ} \mathrm{C}$. After $12 \mathrm{~h}$ the reaction was cooled to room temperature and concentrated in vacuo to yield $3.08 \mathrm{~g}$ (99.7\% yield) of an off white solid that was used without further purification.

II was isolated as a pale yellow solid in an $86 \%$ yield over the two steps. ${ }^{1} \mathrm{H}$
$\mathrm{NMR}\left(500 \mathrm{MHz}, \mathrm{CDCl}_{3}\right) \mathrm{d} 7.56(\mathrm{~m}, 2 \mathrm{H}) ; 7.44(\mathrm{~m}, 3 \mathrm{H}) ; 6.91(\mathrm{~s}, 1 \mathrm{H}) ; 3.97(\mathrm{~s}$,

\section{References:}

(1) Pangborn, A. B.; Giardello, M. A.; Grubbs, R. H.; Rosen, R. K.; Timmers, F. J.

Organometal. 1996, 15, 1518-1520.

(2) Yamamoto, K.; Hayashi, A.; Suzuki, S.; Tsuji, J. Organometal. 1987, 6, 974-979.

(3) Fleming, I.; Ghosh, U. J. Chem. Soc., Perkin Trans. 1 1994, 257-62.

(4) Clark, C. T.; Milgram, B. C.; Scheidt, K. A. Org. Lett. 2004, 6, 3977-3980.

(5) Murphy, W. S.; Wattansin, S. Synthesis 1980, 647-650.

(6) Henrick, C.; Ridley, D.; Ritchie, E.; Taylor, W. Aust. J. Chem. 1970, 23, 329-339.

(7) Horspool, W.; Khalaf, A. J. Chem. Soc. Perkin Trans. 1 1989, 1147-1152.

(8) Nagarajan, M.; Shechter, H. J. Org. Chem. 1984, 49, $62-74$.

(9) Stetter, H.; Schreckenberg, M. Chem. Ber. 1974, 107, 2453-2458.

(10) Zdrojewski, T.; Jonczyk, A. Synthesis 1990, 224-233.

(11) Hegedus, L. S.; Perry, R. J. J. Org. Chem. 1985, 50, 4955-4960.

(12) Yus, M.; Ortiz, J.; Najera, C. Arkivoc 2002, 38-47.

(13) Saigo, K.; Kurihara, H.; Miura, H.; Hongu, A.; Kubota, N.; Nohira, H.; Hasegawa, M. Syn. Comm. 1984, 14, 787-796.

(14) Kawenoki, I.; Maurel, D.; Kossanyi, J. Bull. Soc. Chim. Fr. 1982, 385-390.

(15) Rieke, R. D.; Stack, D. E.; Dawson, B. T.; Wu, T. C. J. Org. Chem. 1993, 58, 2483-2491.

(16) Yasuda, M.; Nishio, M.; Shibata, I.; Baba, A.; Matsuda, H. J. Org. Chem. 1994, 59, 486487.

(17) Furstner, A.; Jumbam, D. N. Tetrahedron 1992, 48, 5991-6010.

(18) Lee, C. F.; Yang, L. M.; Hwu, T. Y.; Feng, A. S.; Tseng, J. C.; Luh, T. Y. J. Am. Chem. Soc. 2000, 122, 4992-4993.

(19) Brown, C. D.; Chong, J. M.; Shen, L. X. Tetrahedron 1999, 55, 14233-14242.

(20) Stetter, H.; Schmitz, P. H.; Schreckenberg, M. Chem. Ber. 1977, 110, 1971-1977.

(21) Furstner, A.; Weintritt, H.; Hupperts, A. J. Org. Chem. 1995, 60, 6637-6641. 
(22) Armesto, D.; Horspool, W. M.; Ortiz, M. J.; Romano, S. J. Chem. Soc., Perkin Trans. 1 1992, 171-175.

(23) Farcas, S.; Namy, J. L. Tetrahedron 2001, 57, 4881-4888.

(24) Yoshida, H.; Utsumi, F.; Suzuki, H.; Ito, S.; Sakashita, S.; Ogata, T.; Matsumoto, K. Bull. Chem. Soc. Jpn. 1992, 65, 698-702. 


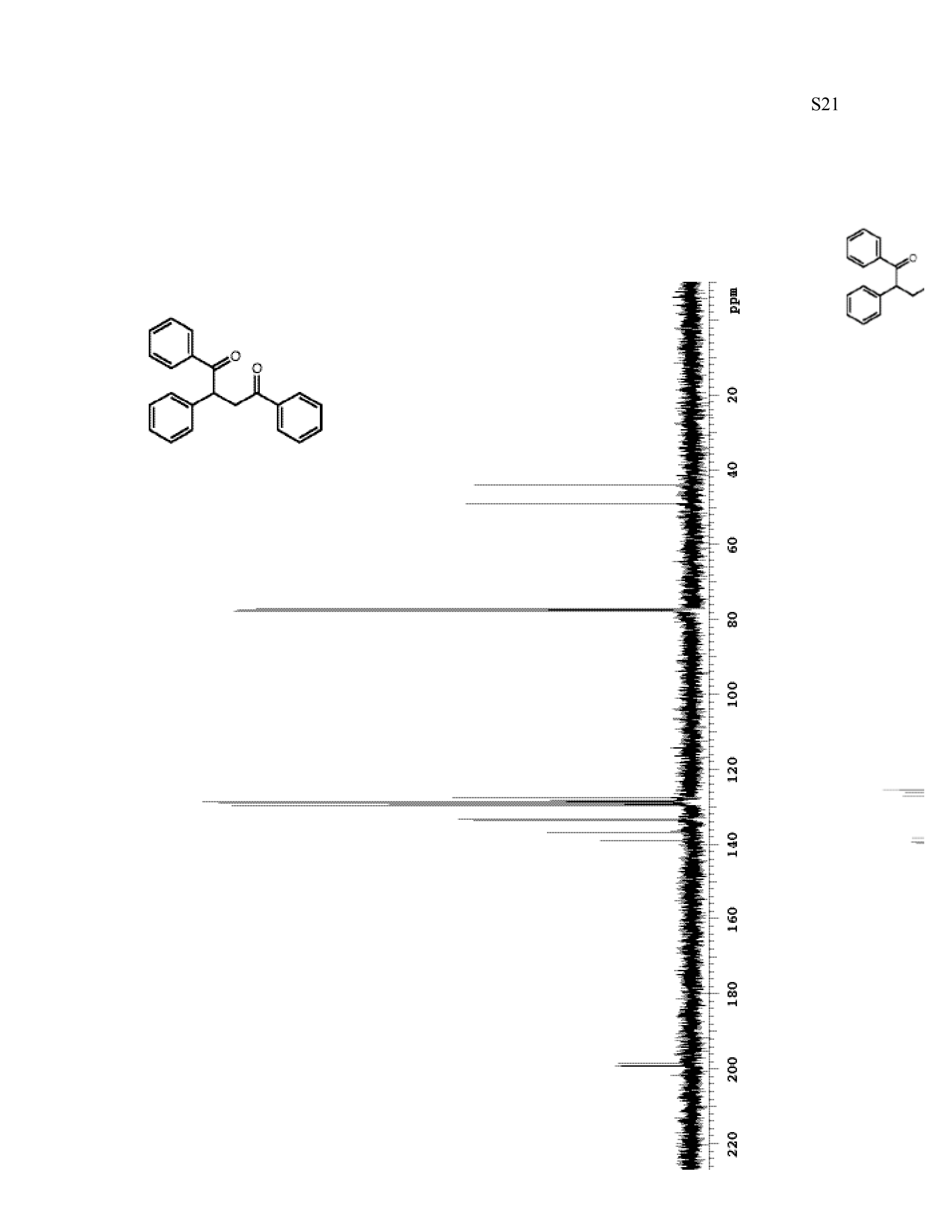


<smiles>O=C(CC(C(=O)CC(C(=O)c1ccccc1)c1ccccc1)c1ccc(Cl)cc1)c1ccc(Cl)cc1</smiles>

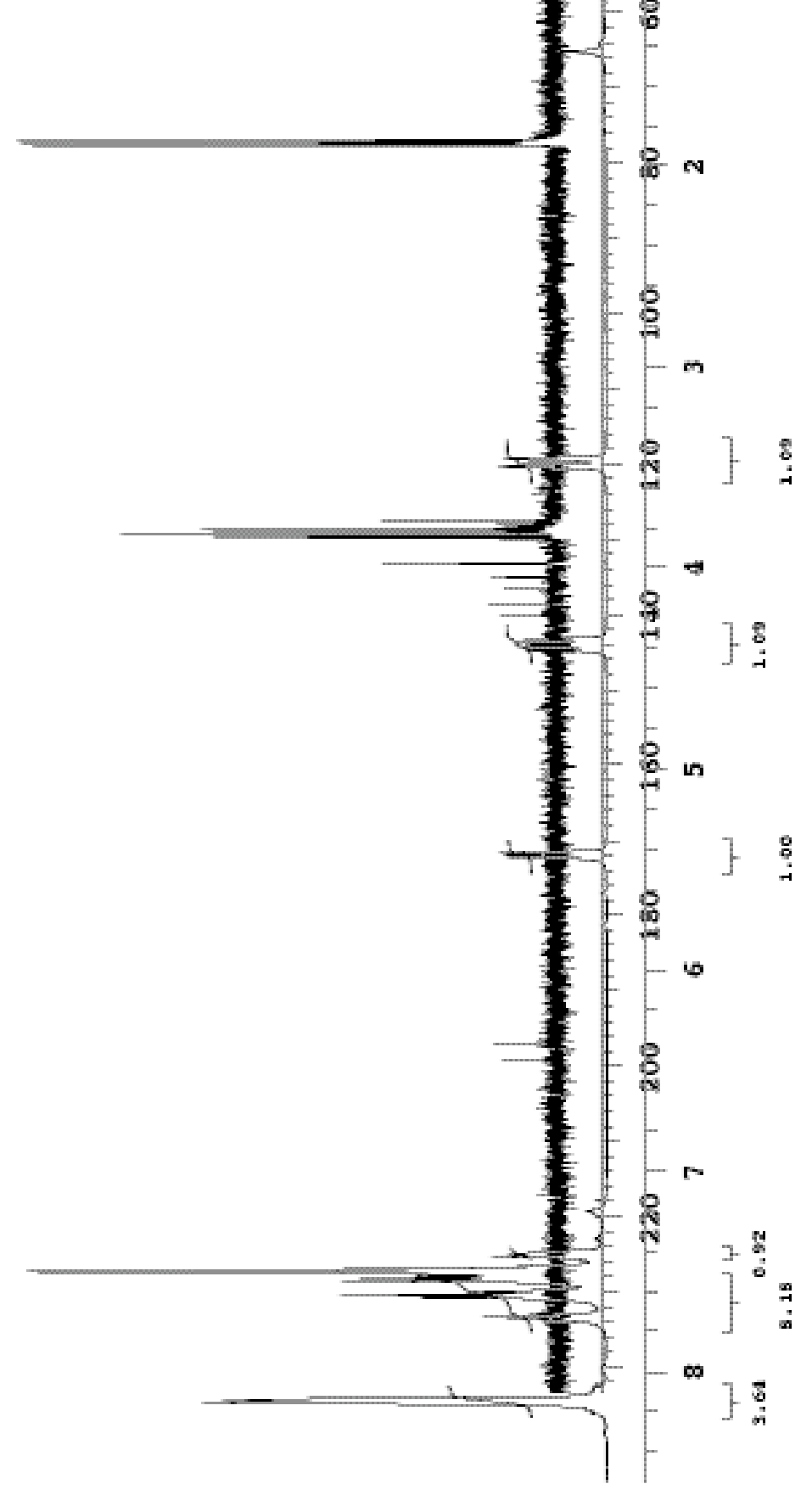


<smiles>COc1ccc(C(=O)CC(C(=O)c2ccccc2)c2ccccc2)cc1</smiles>

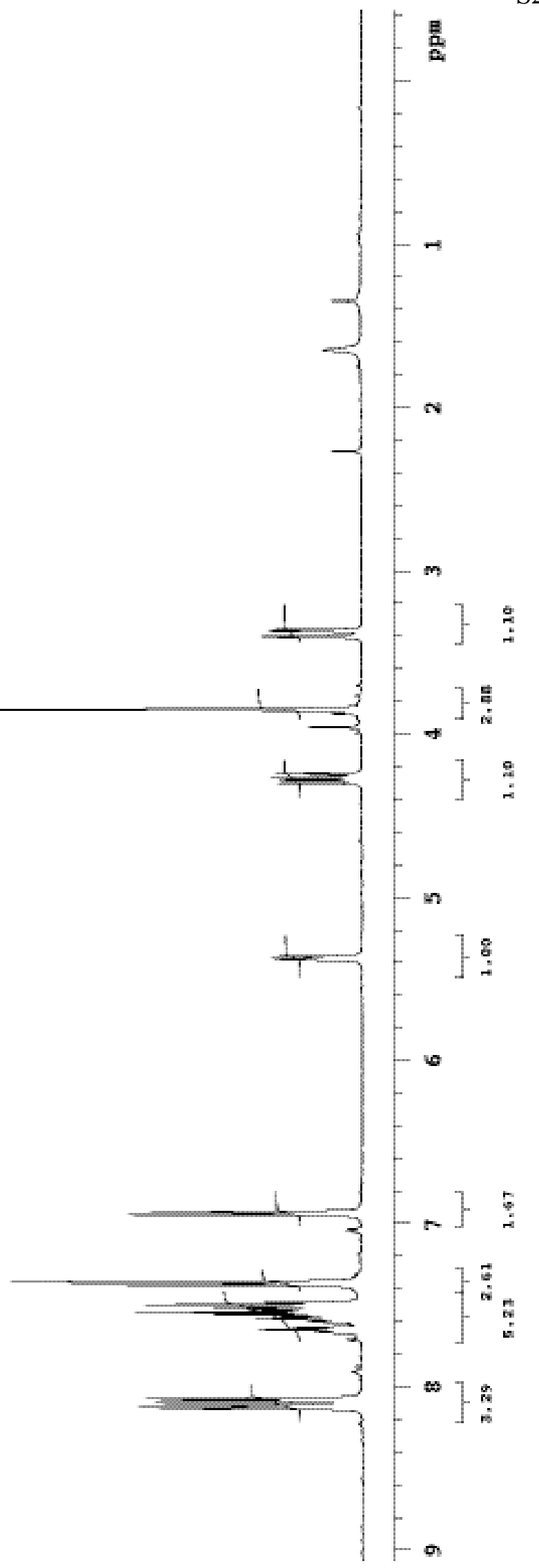




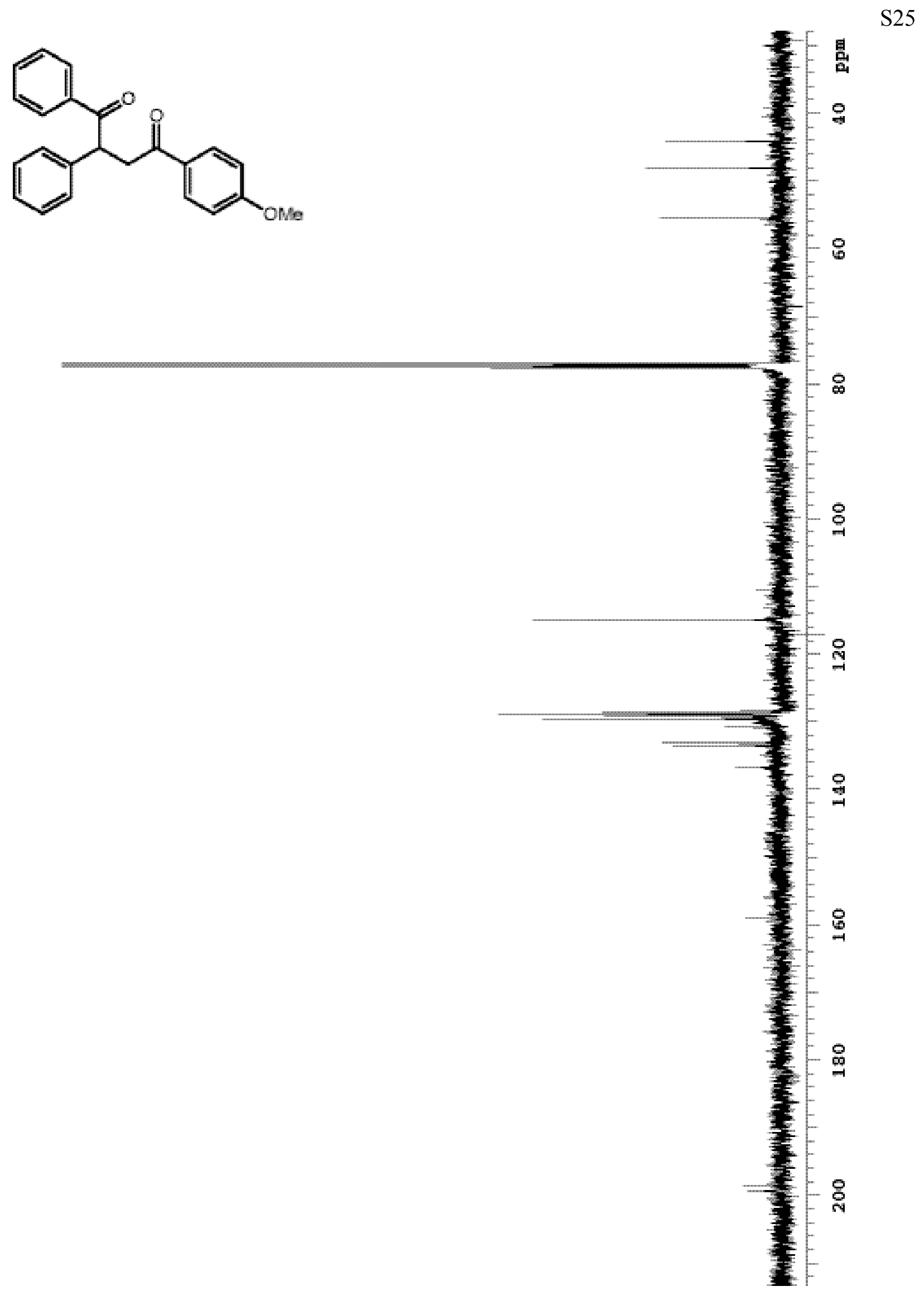


<smiles>O=C(CC(C(=O)c1ccccc1)c1cccc2ccccc12)c1ccccc1</smiles>

1 - 음

品
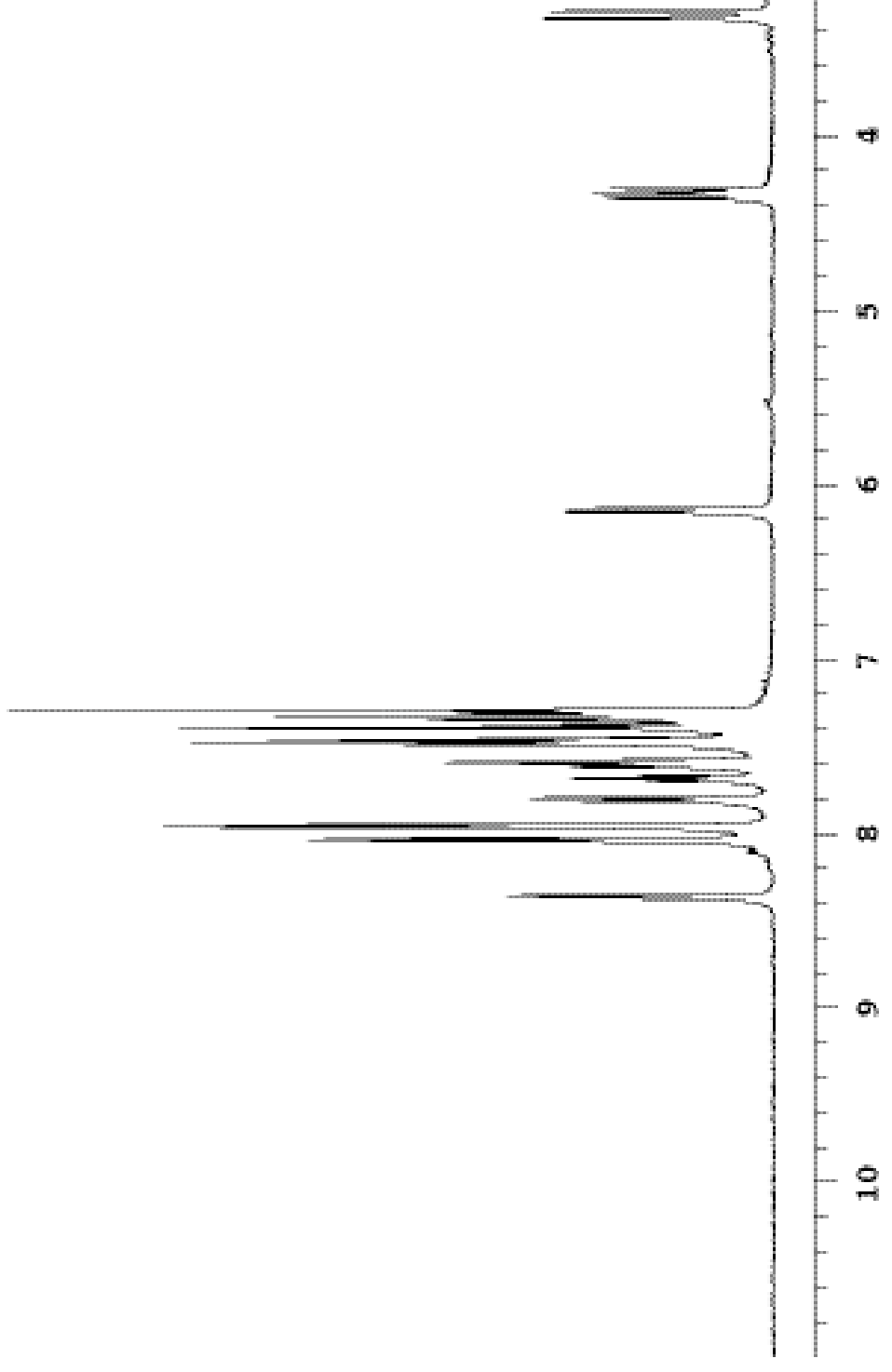


$$
4
$$


S28<smiles>O=C(CC(C(=O)c1ccccc1)c1ccc(Br)cc1)c1ccccc1</smiles> 


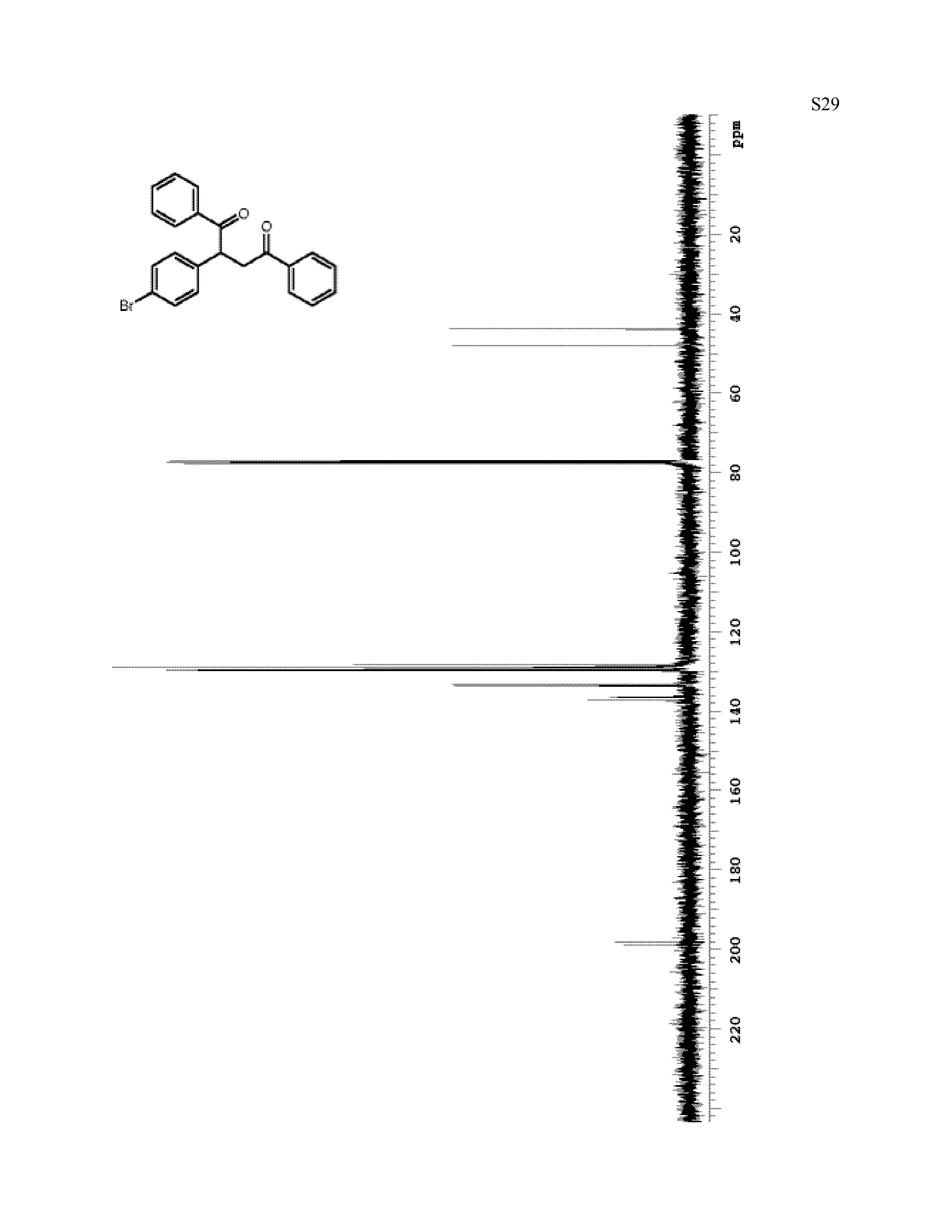


<smiles>C1=C[GeH2][GeH2]1</smiles>

S30

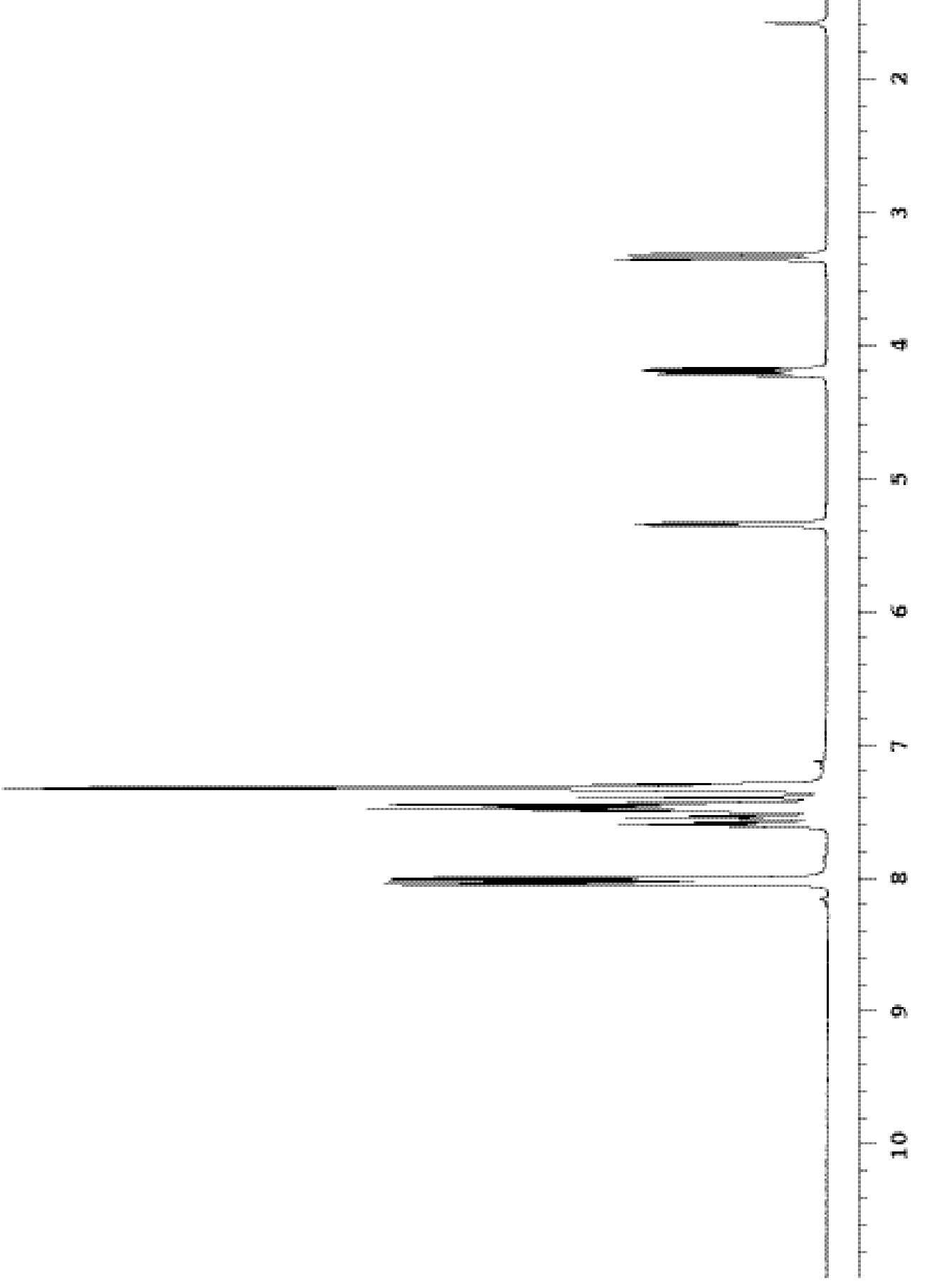



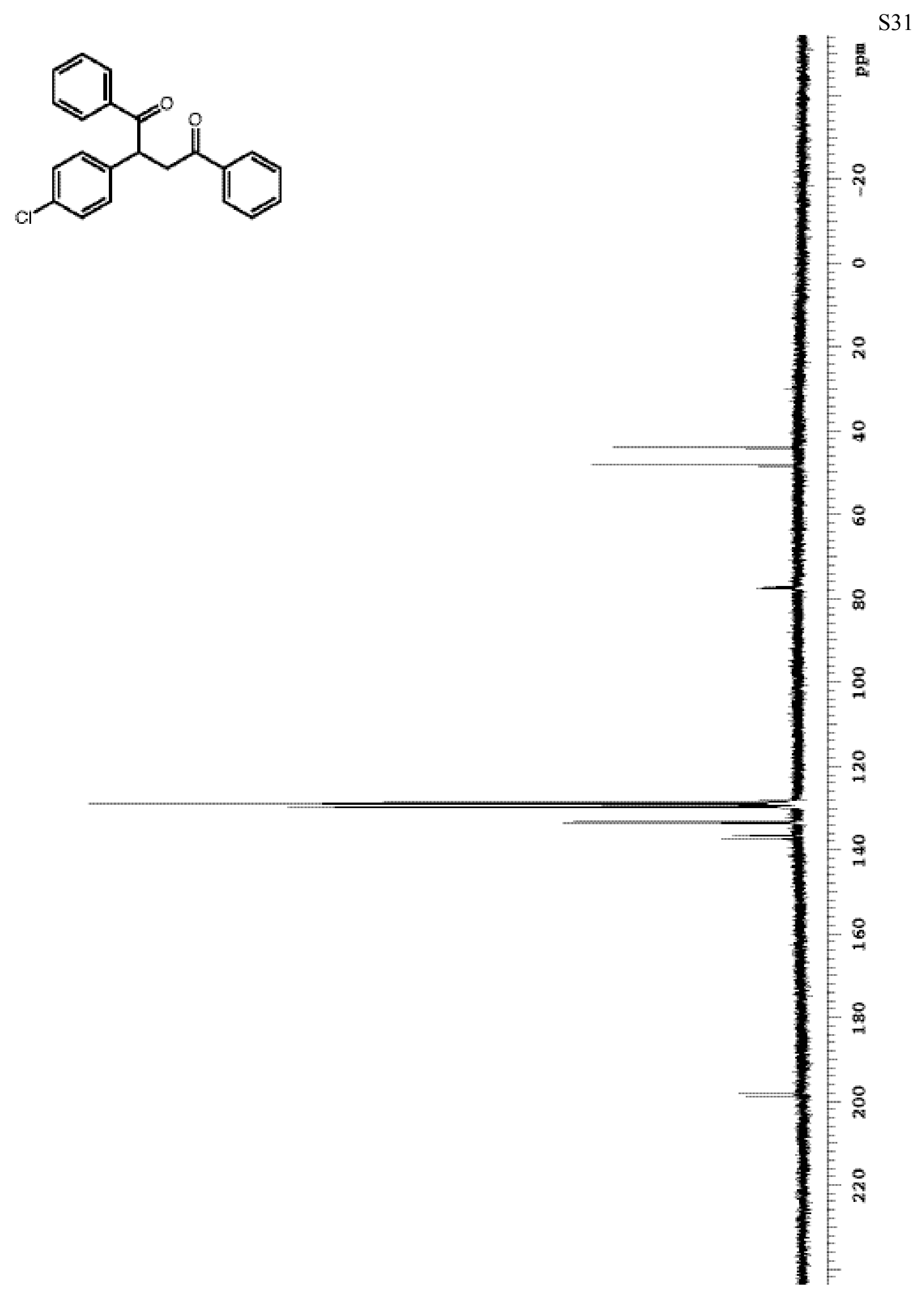


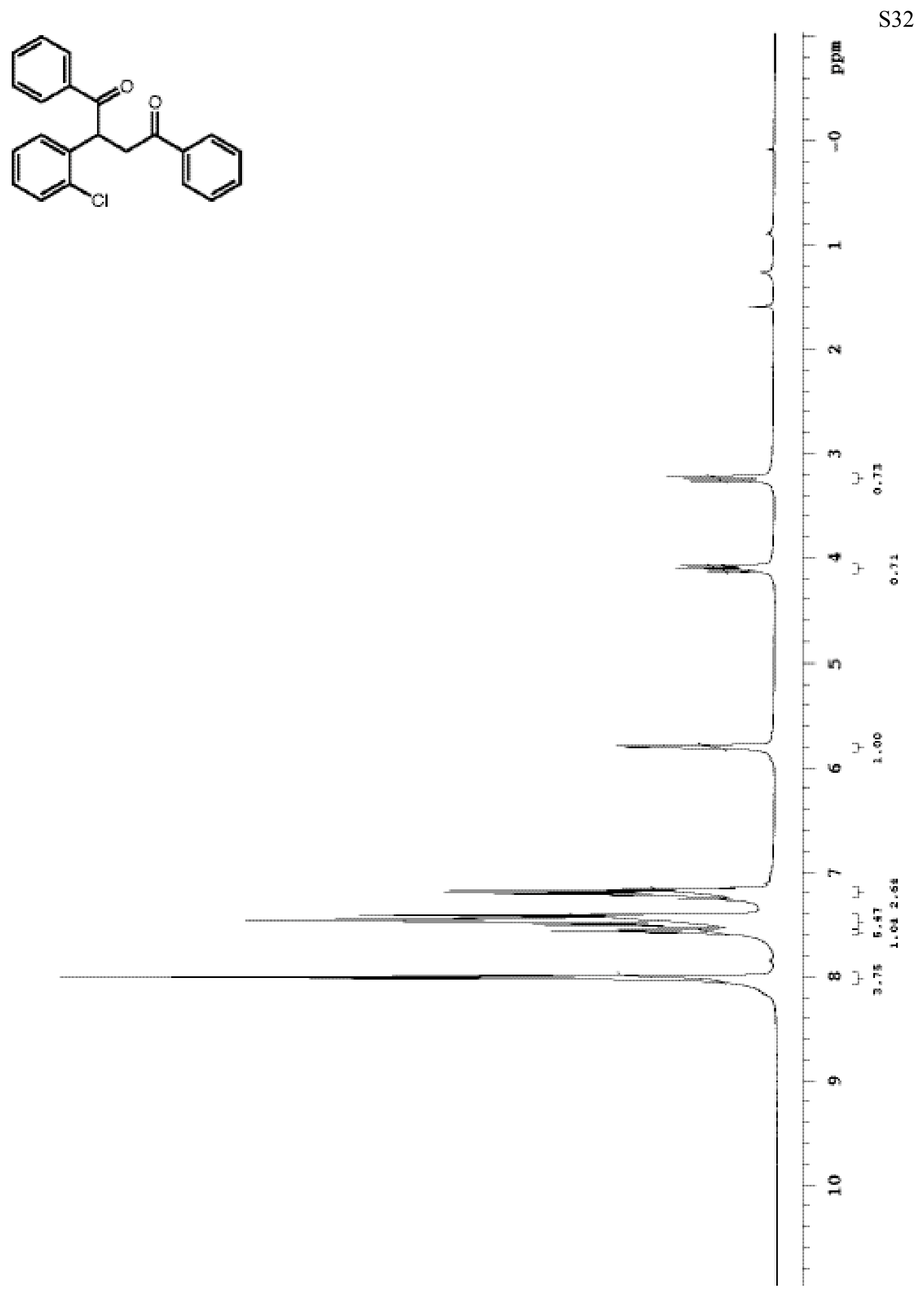




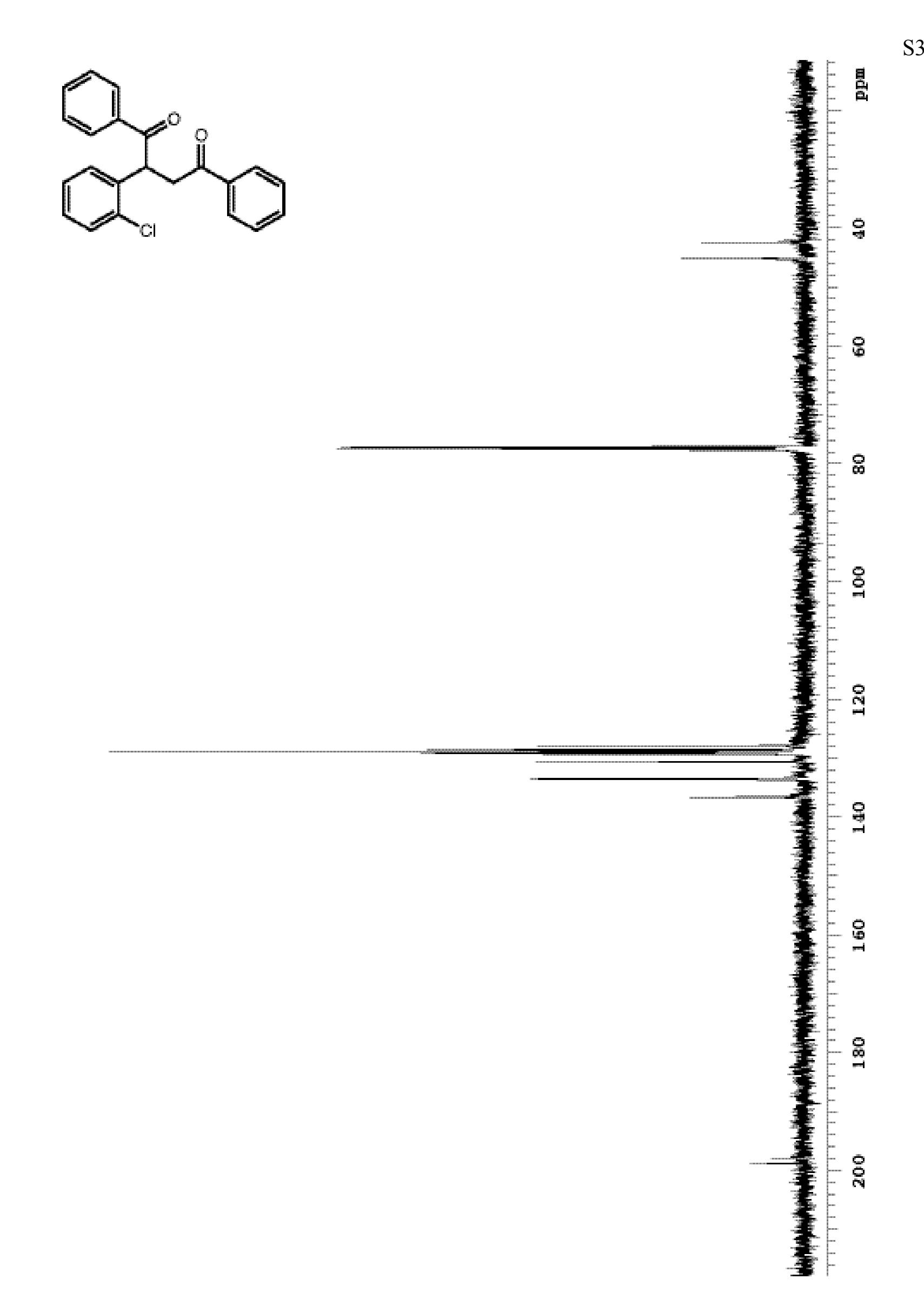



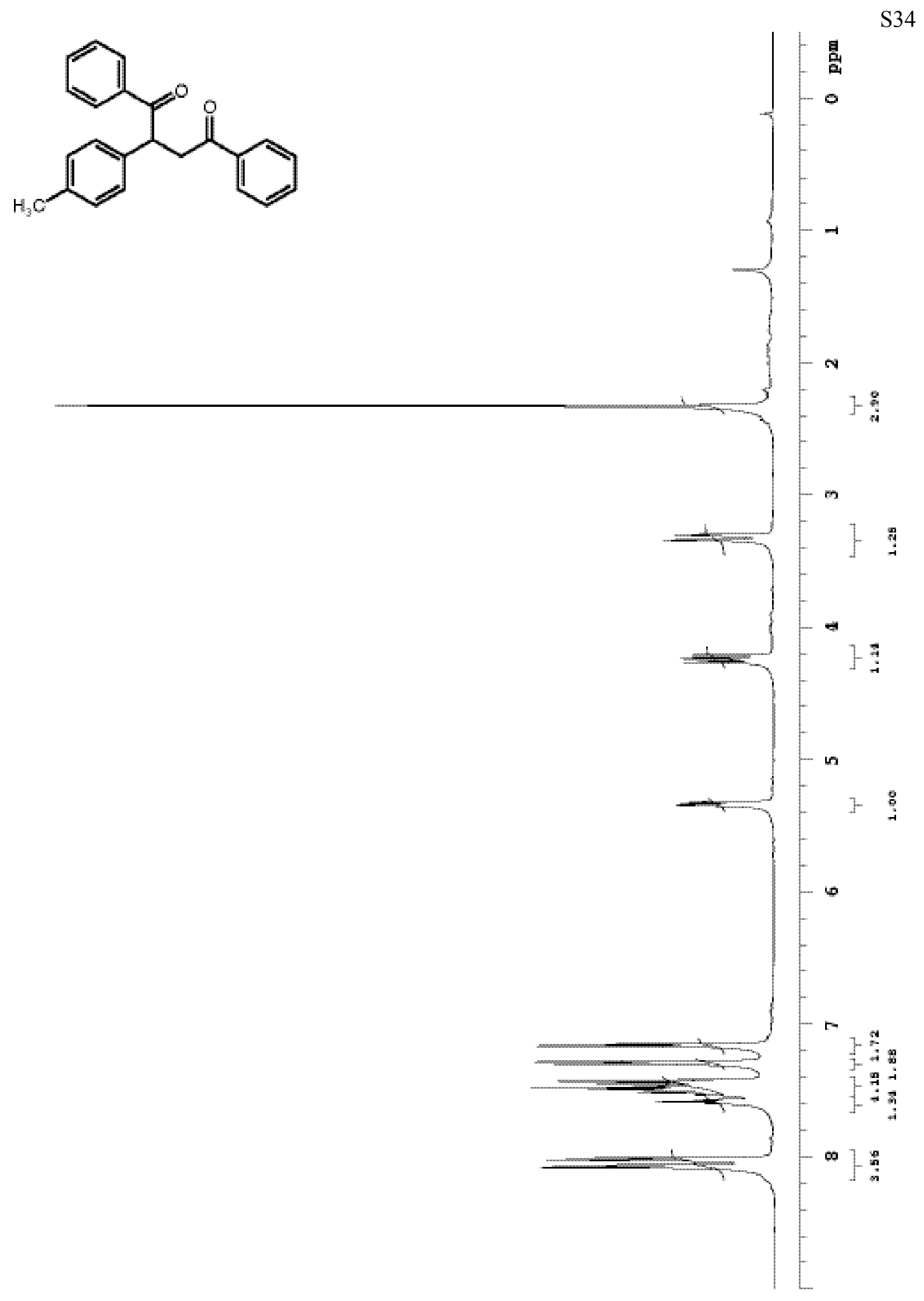


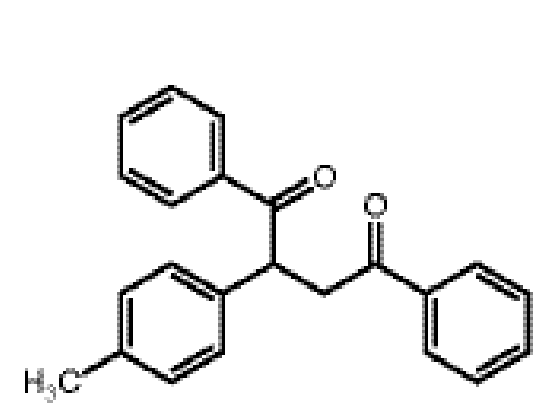

S35

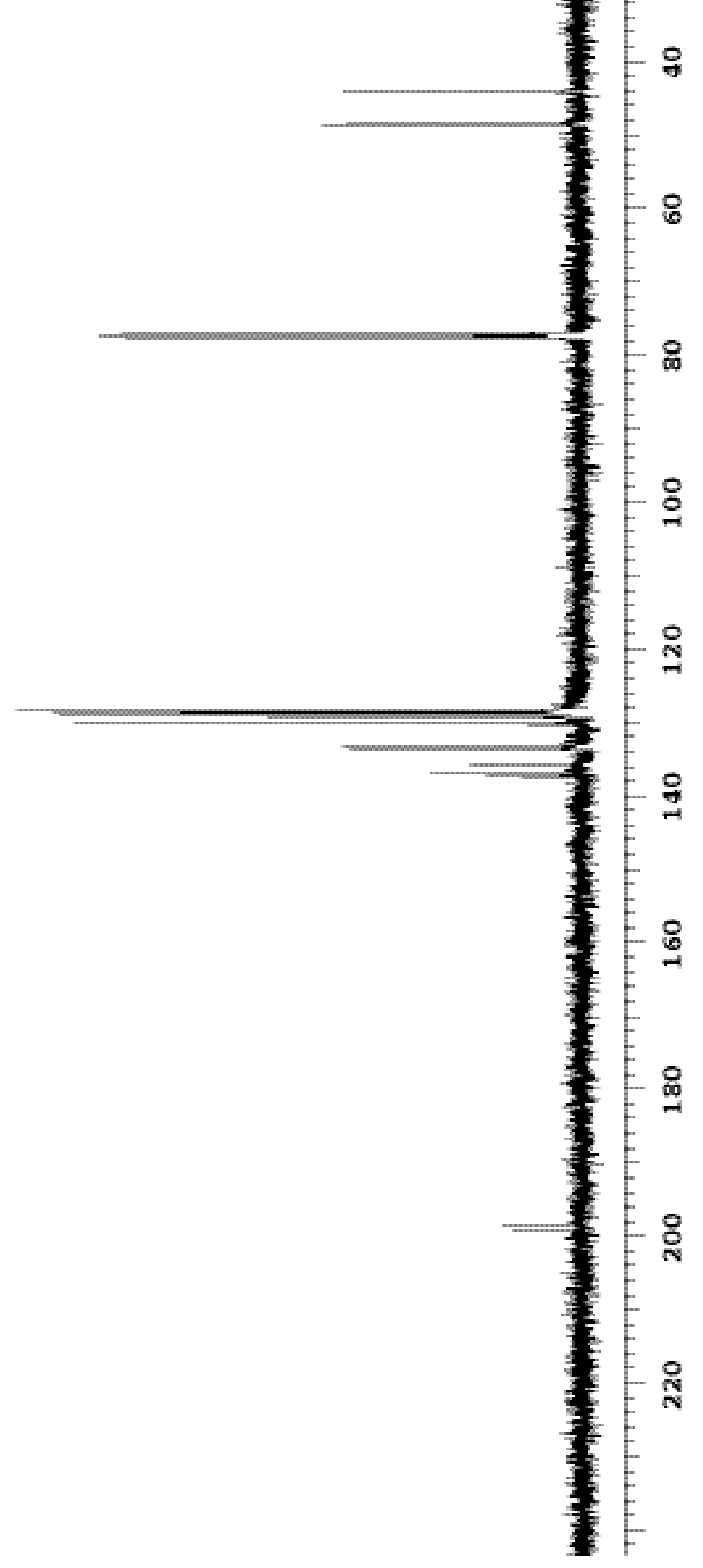


<smiles>COc1cccc(C(CC(=O)c2ccccc2)C(=O)c2ccccc2)c1</smiles>

S36

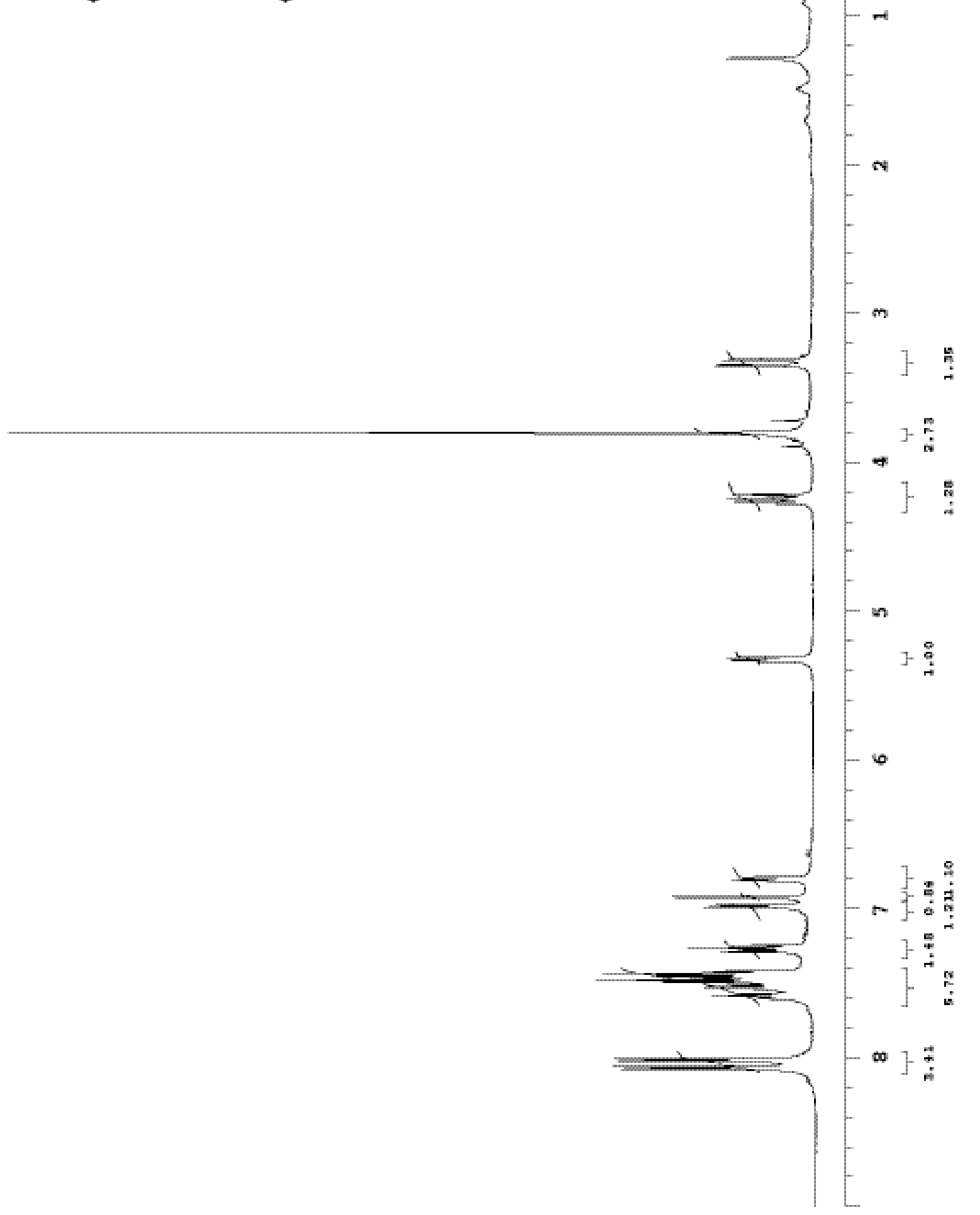


<smiles>COc1cccc(C(CC(=O)c2ccccc2)C(=O)c2ccccc2)c1</smiles>

S37

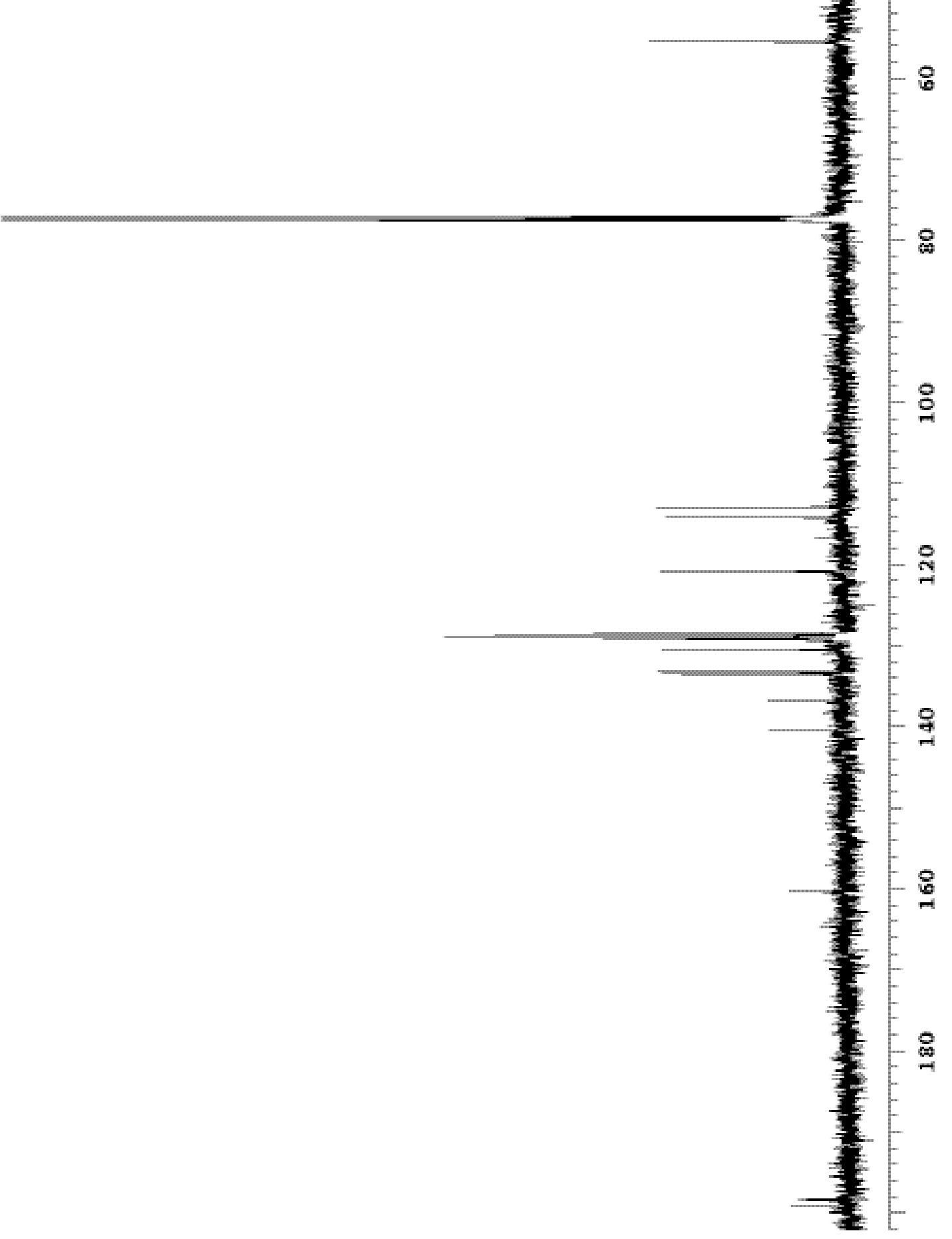


<smiles>COc1ccc(C(CC(=O)c2ccccc2)C(=O)c2ccccc2)cc1</smiles>

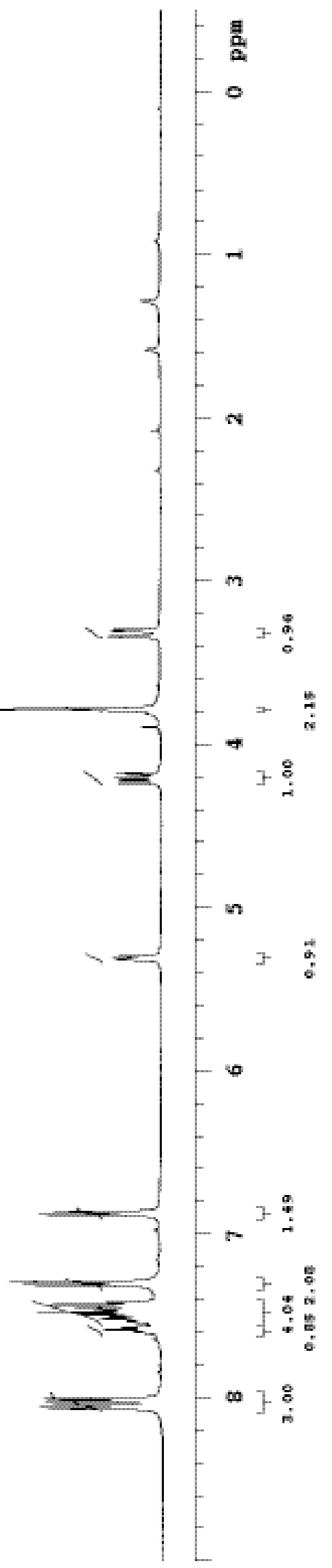


<smiles>COc1ccc(C(CC(=O)c2ccccc2)C(=O)c2ccccc2)cc1</smiles>

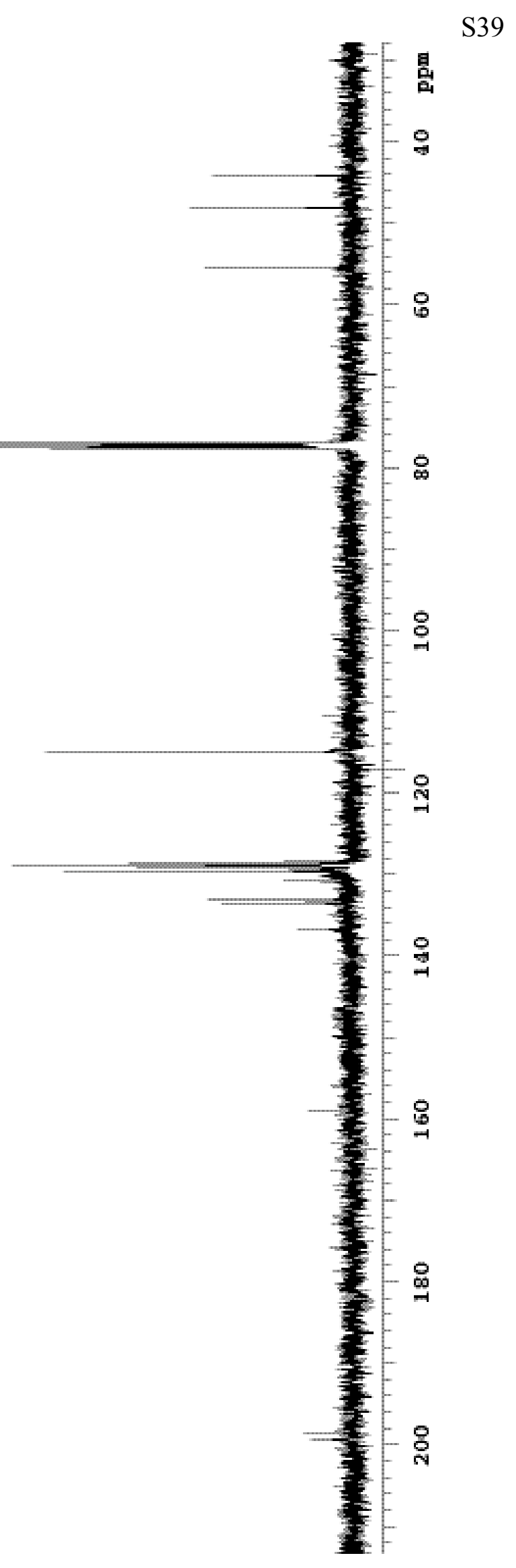



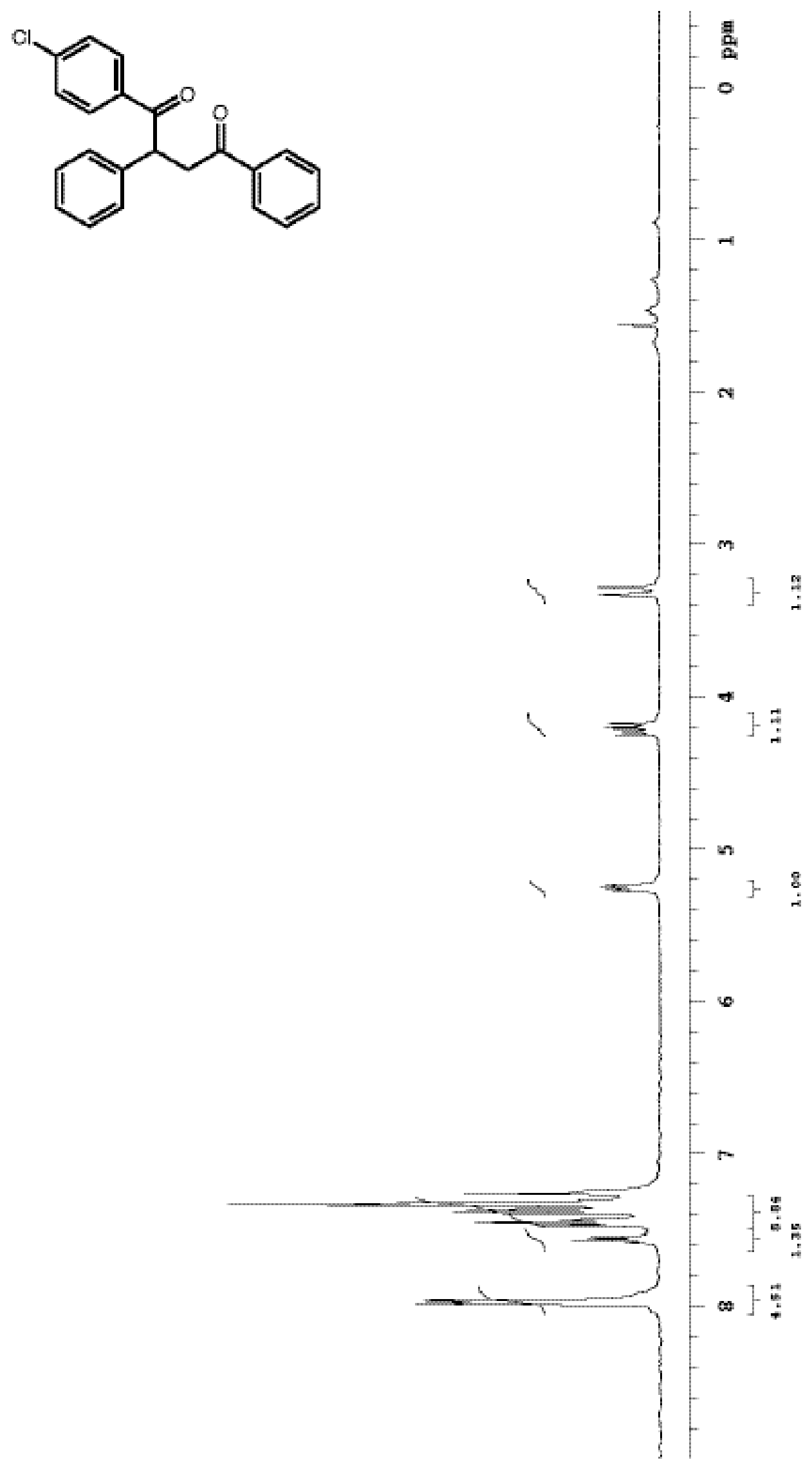


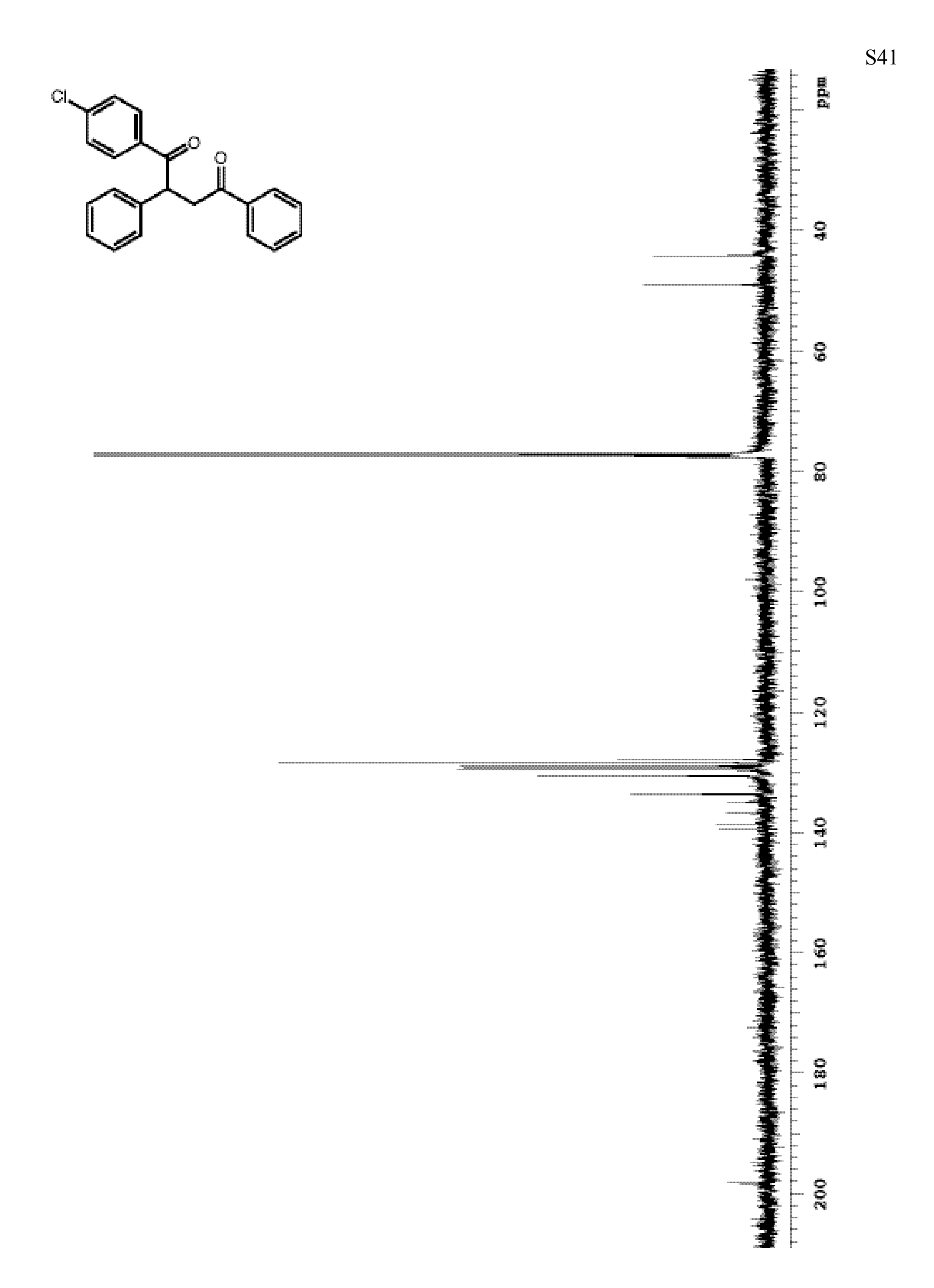




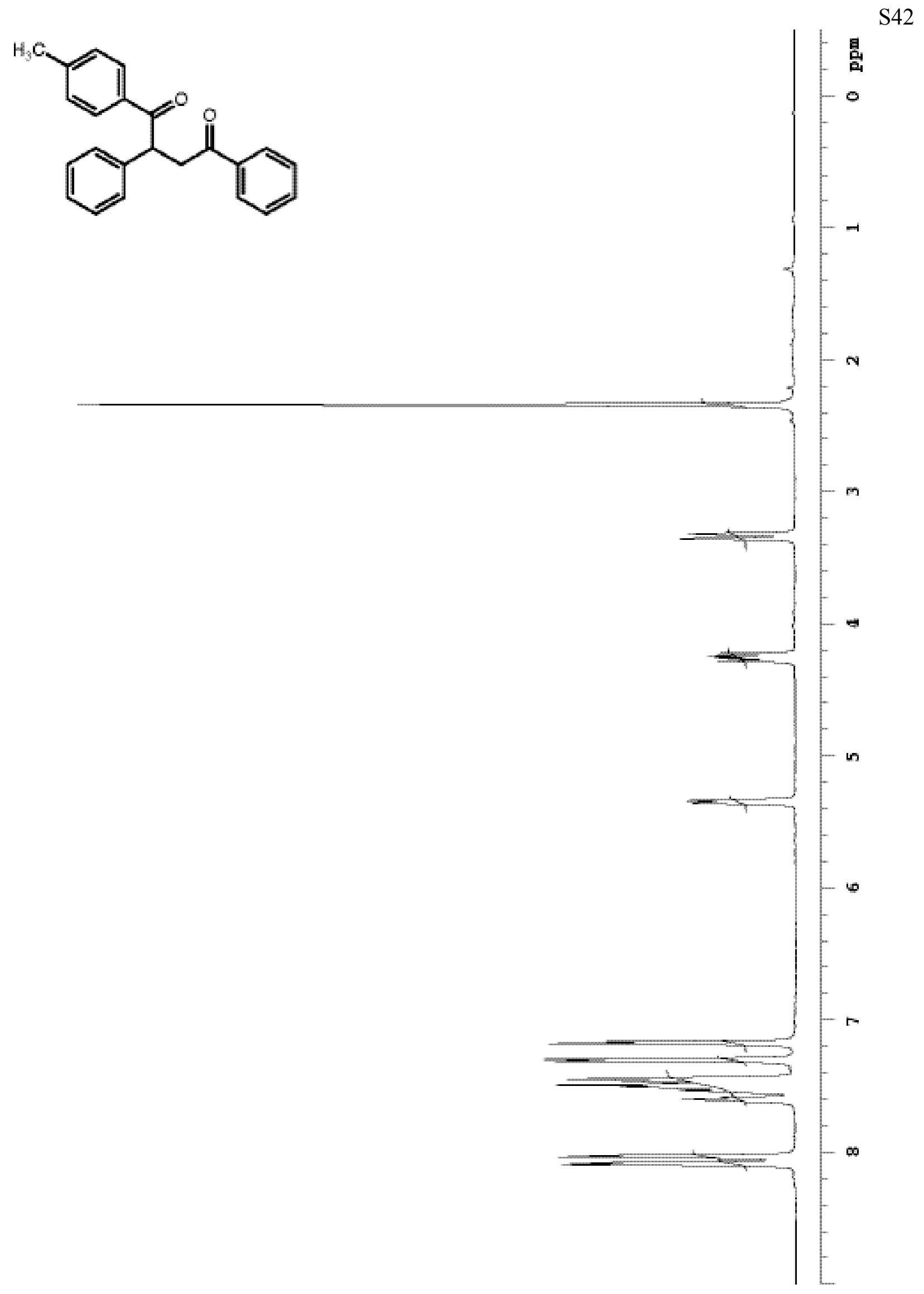


<smiles>Cc1ccc(C(=O)C(CC(=O)c2ccccc2)c2ccccc2)cc1</smiles>

S43

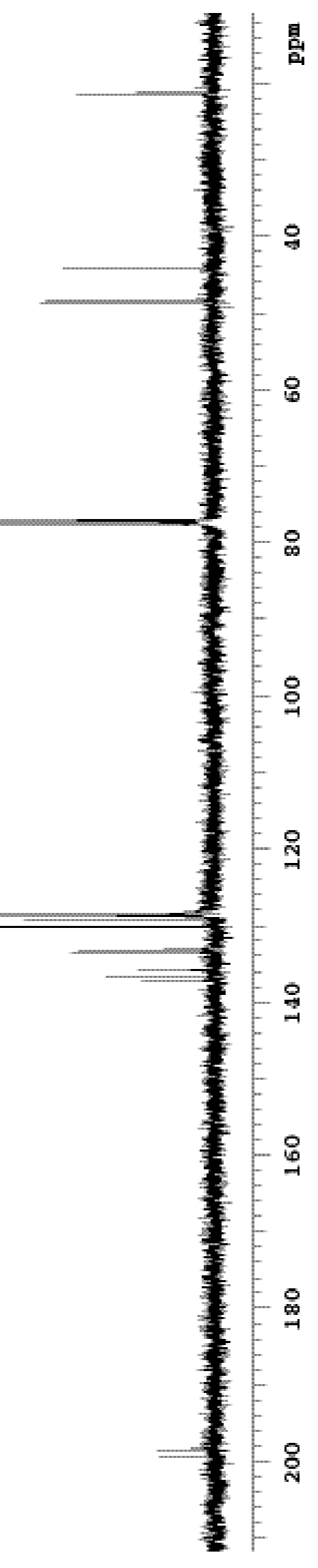




$$
\text { orio }
$$




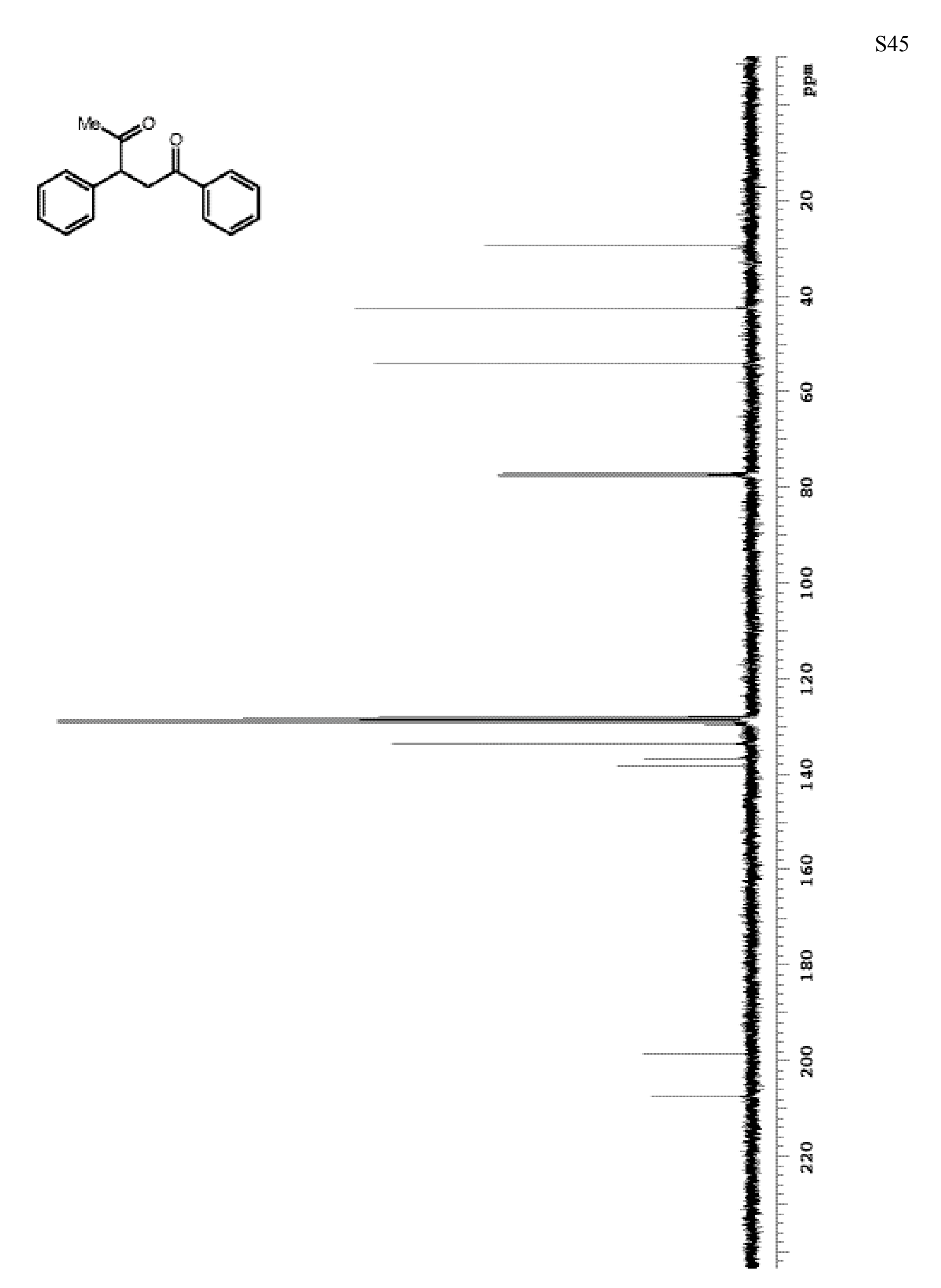


<smiles>O=C(CC(C(=O)C1CCCCC1)c1ccccc1)c1ccccc1</smiles>
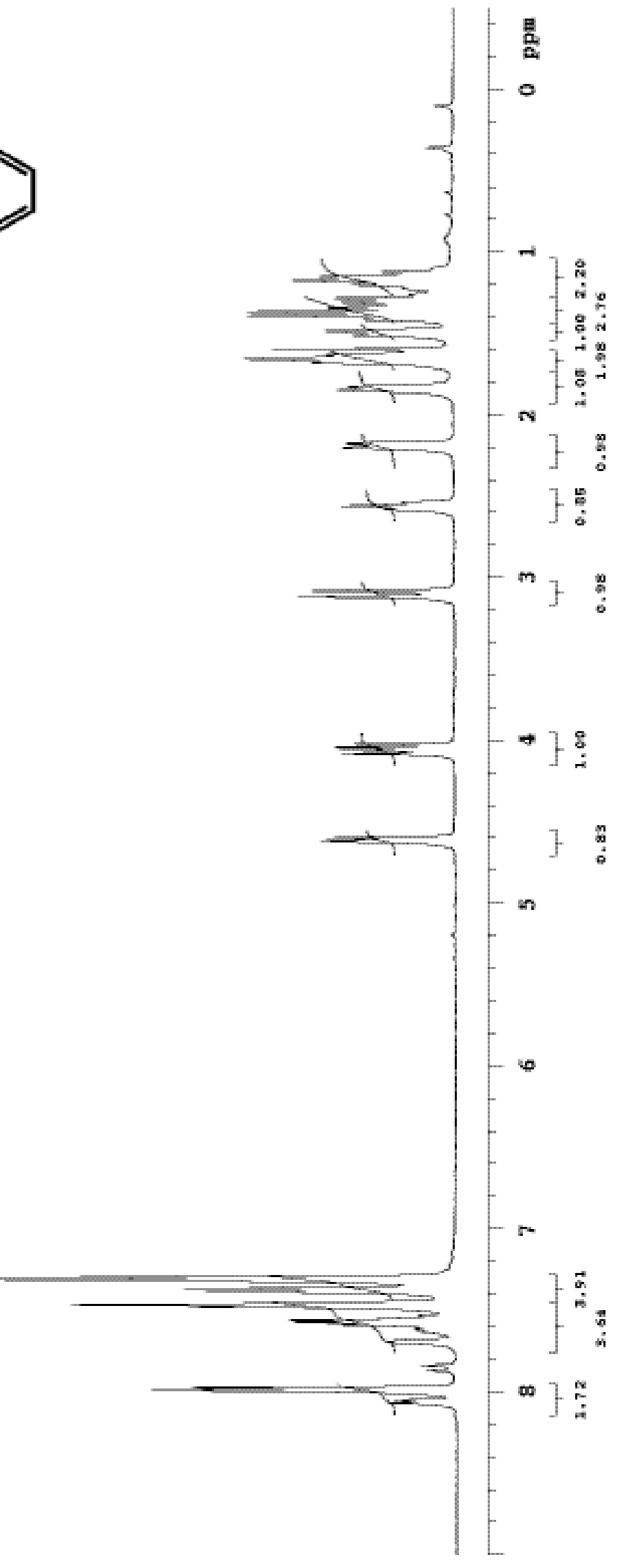
<smiles>CCOC(=O)CC(C(=O)OCC)C(=O)c1ccccc1</smiles>

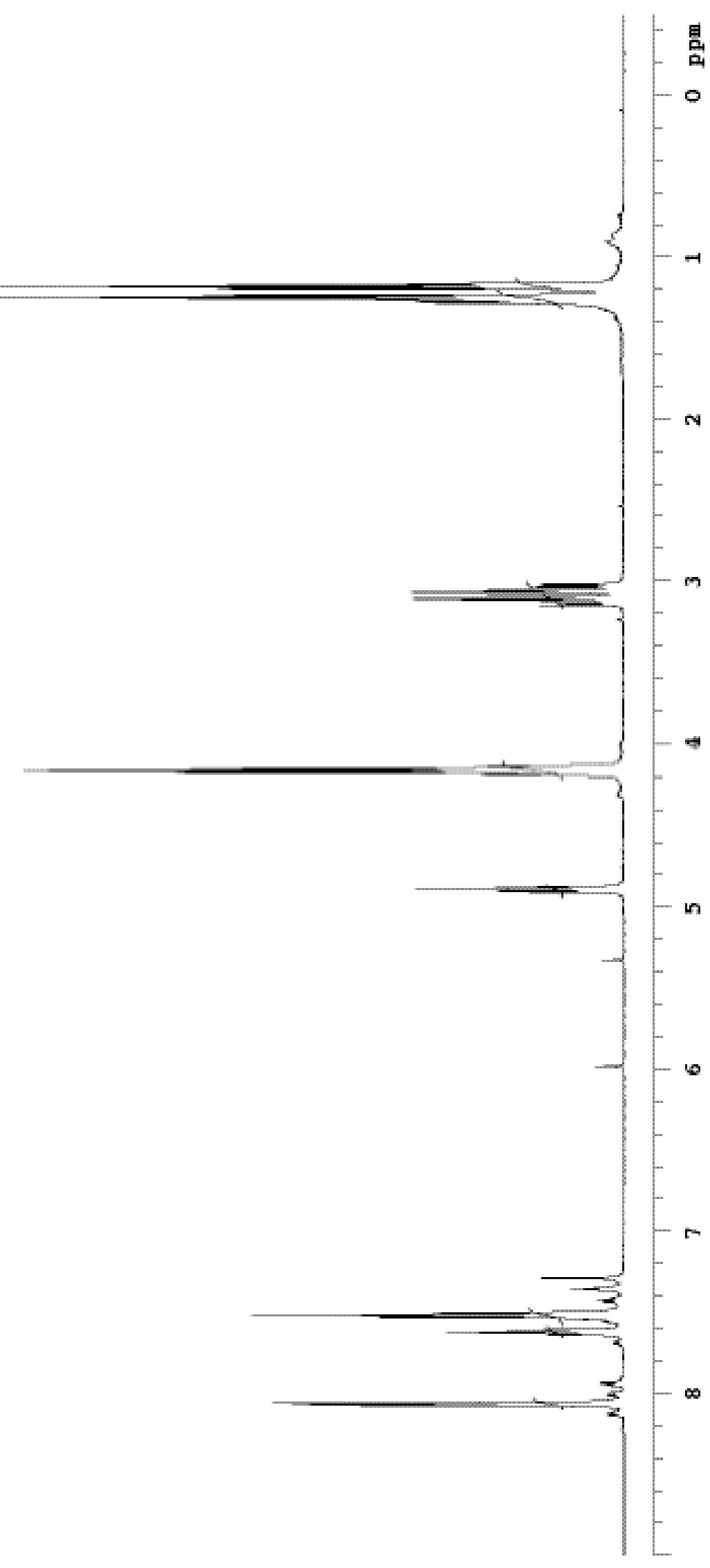




$$
1
$$



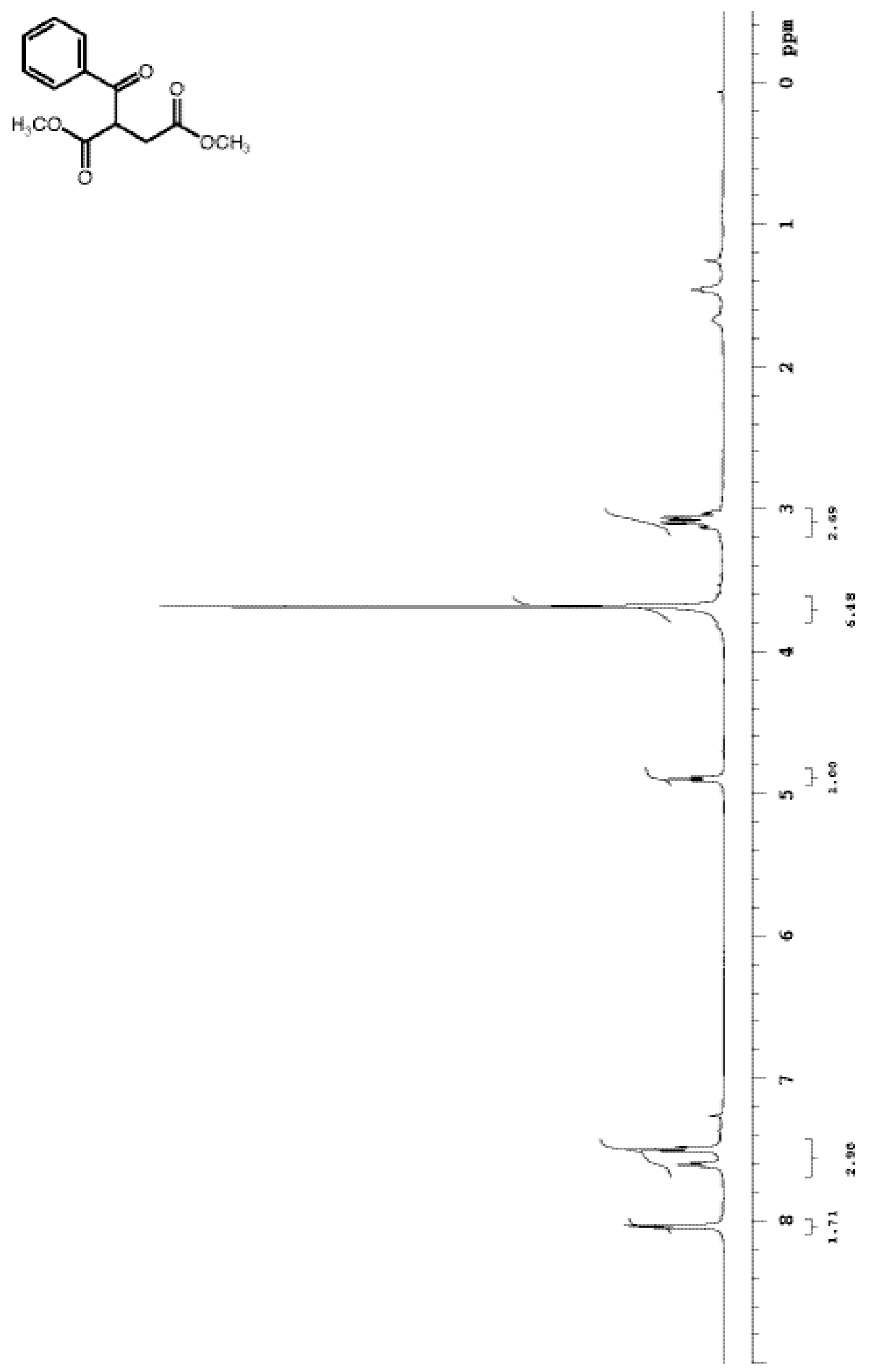


$$
1
$$




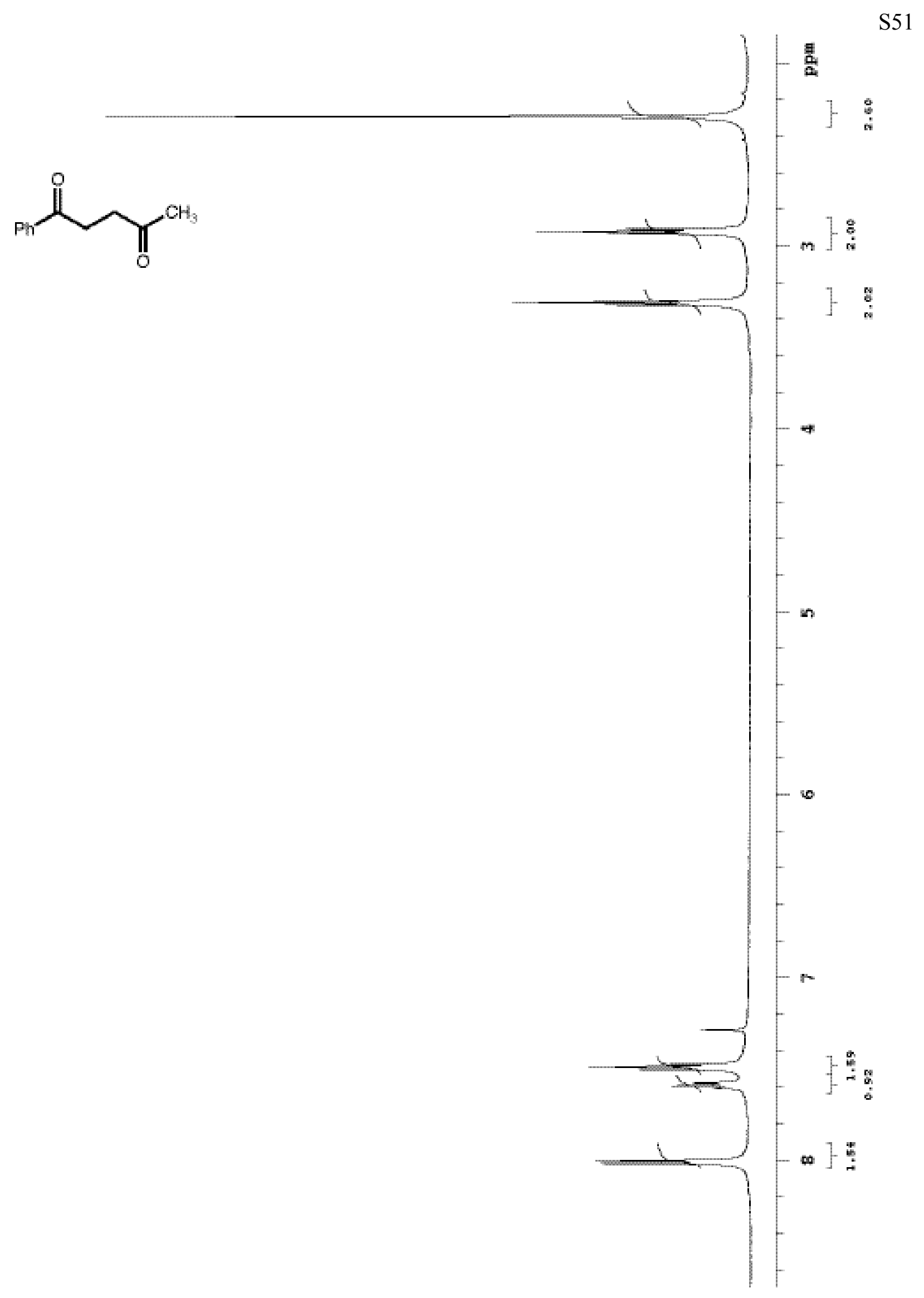


<smiles>CC(=O)CCC(=O)c1ccccc1</smiles>

S52

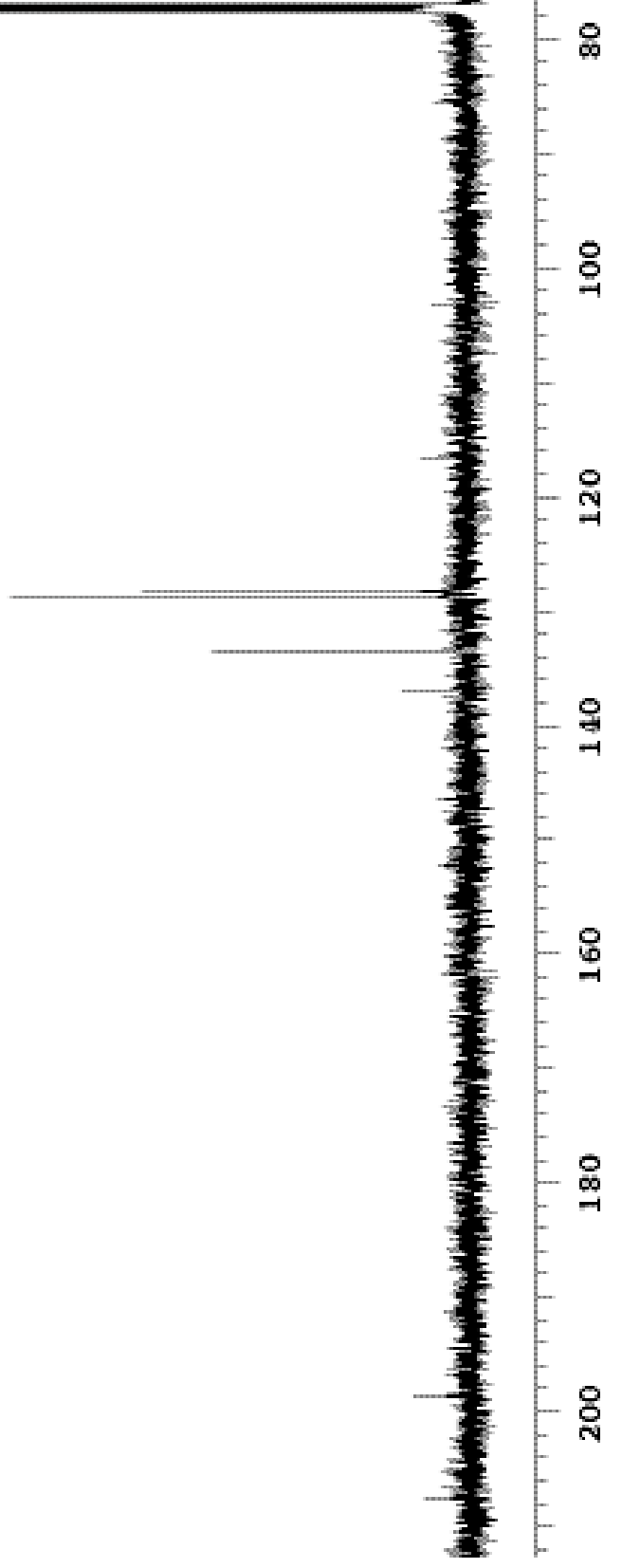




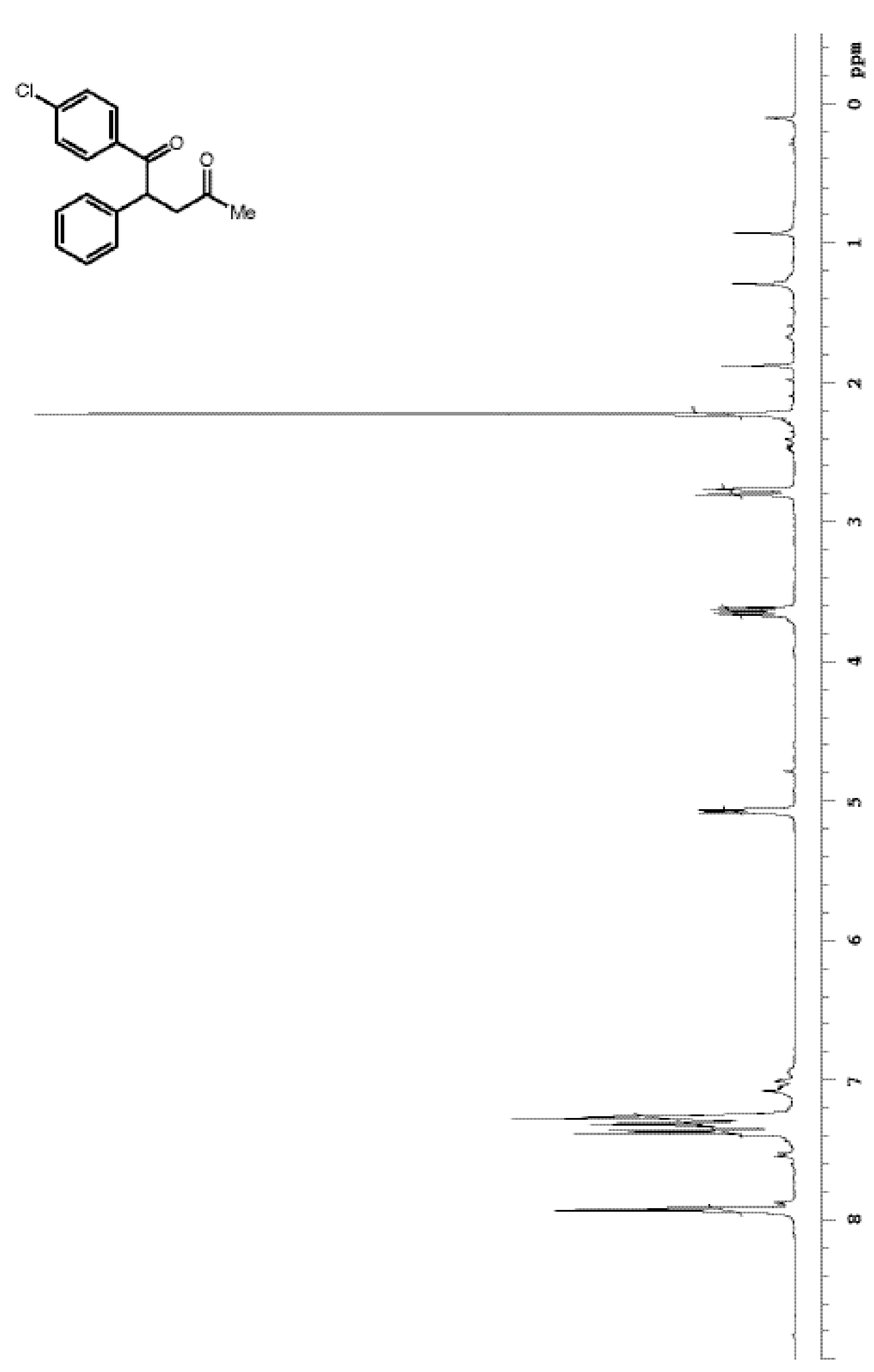


<smiles>CC(=O)CC(C(=O)c1ccc(Cl)cc1)c1ccccc1</smiles>

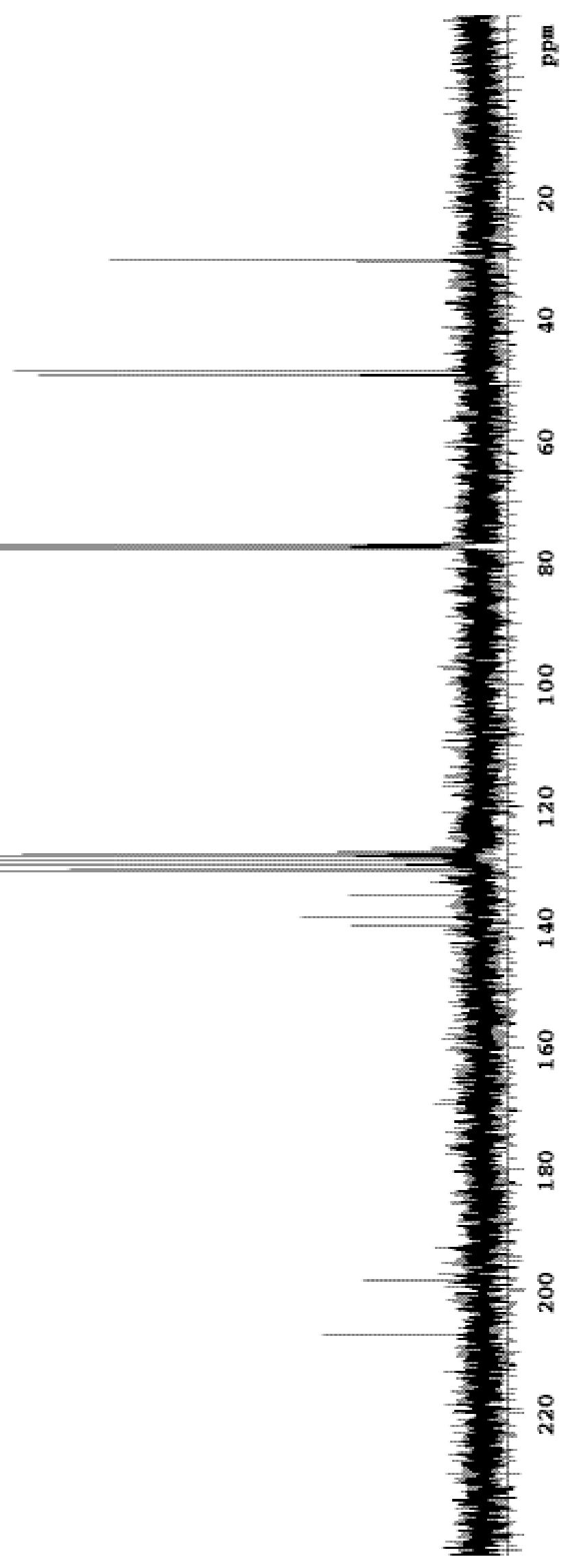



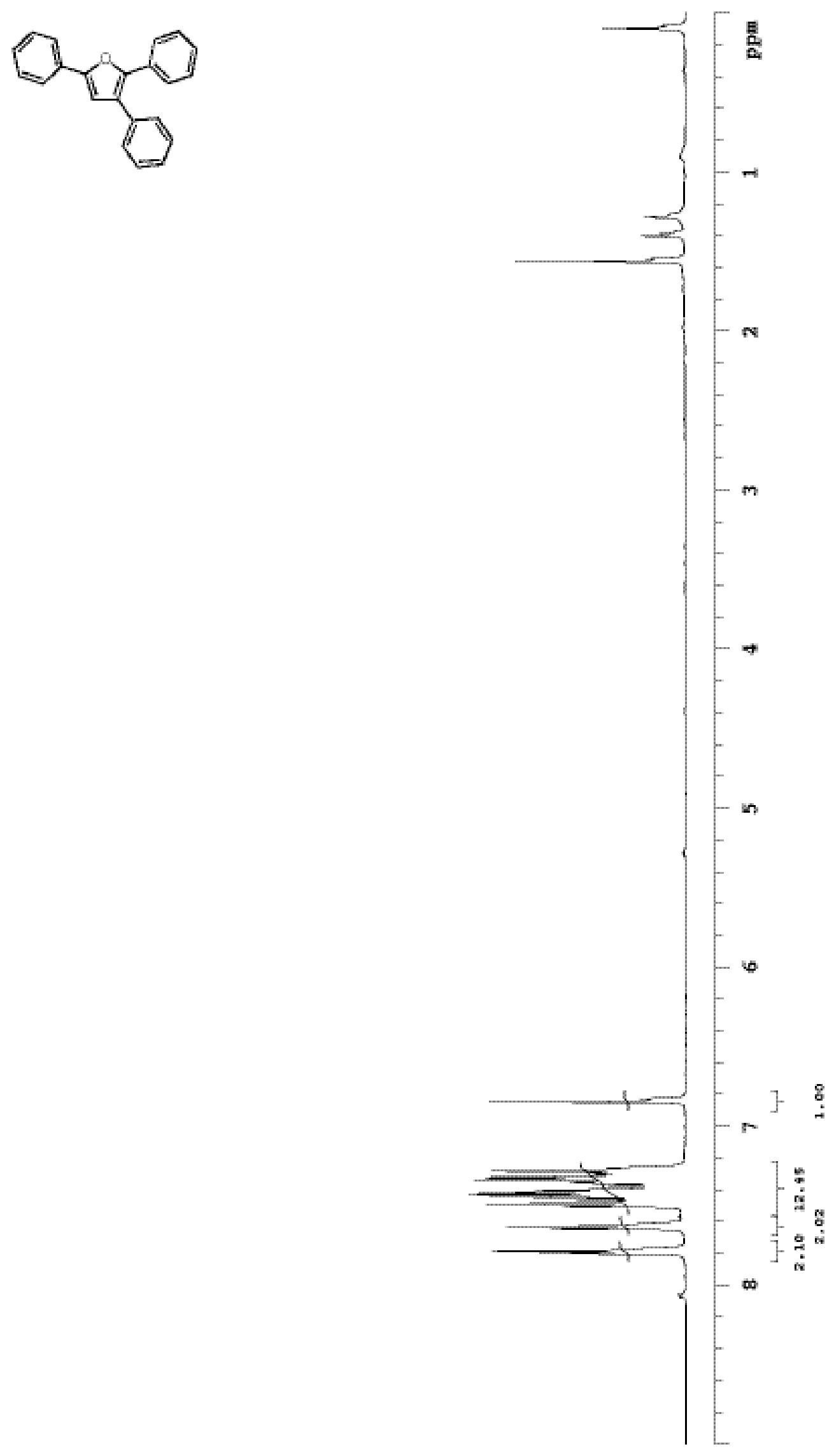


$$
4
$$




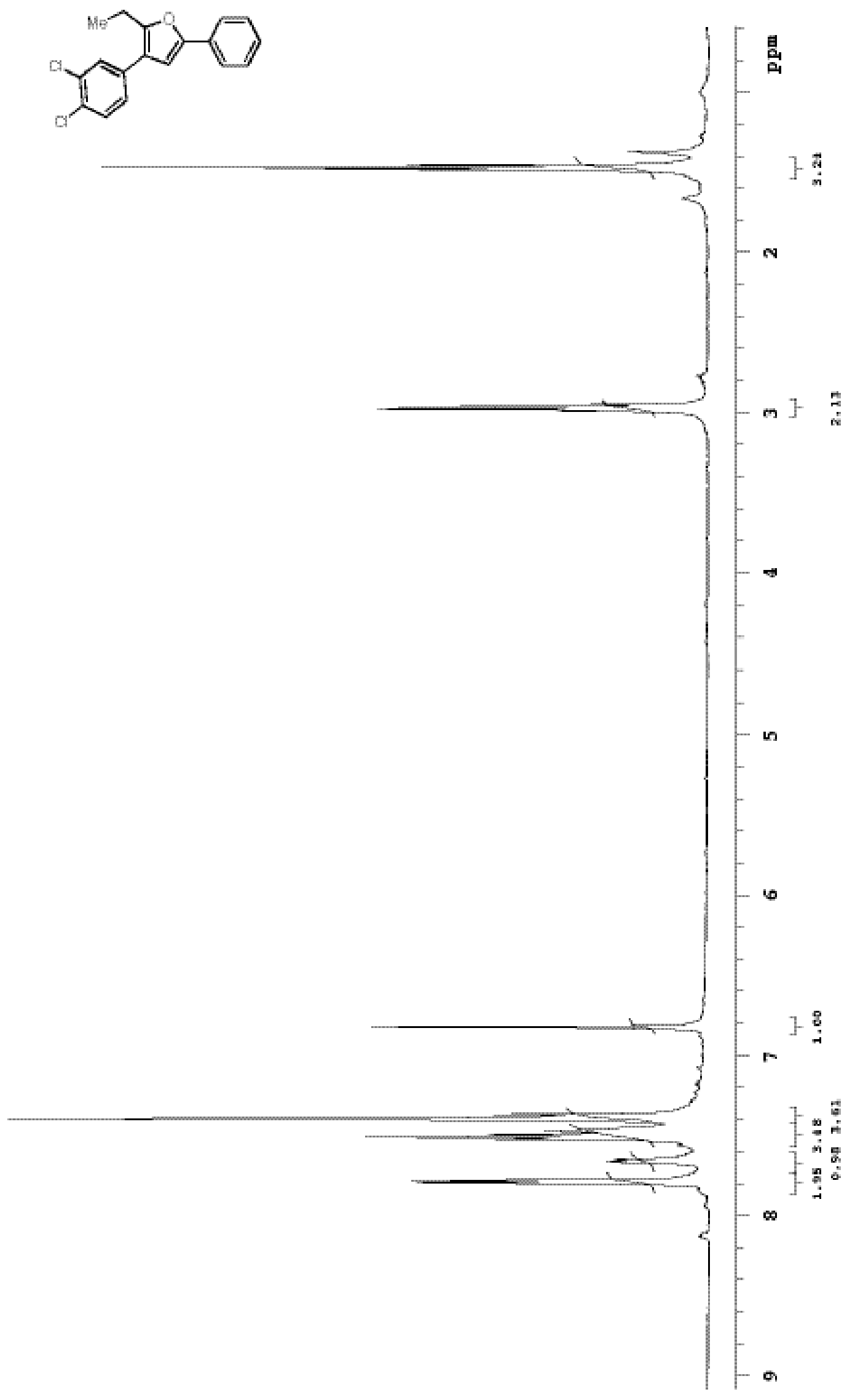




$$
4
$$




$$
\text { A }
$$




$$
\exists
$$


S61
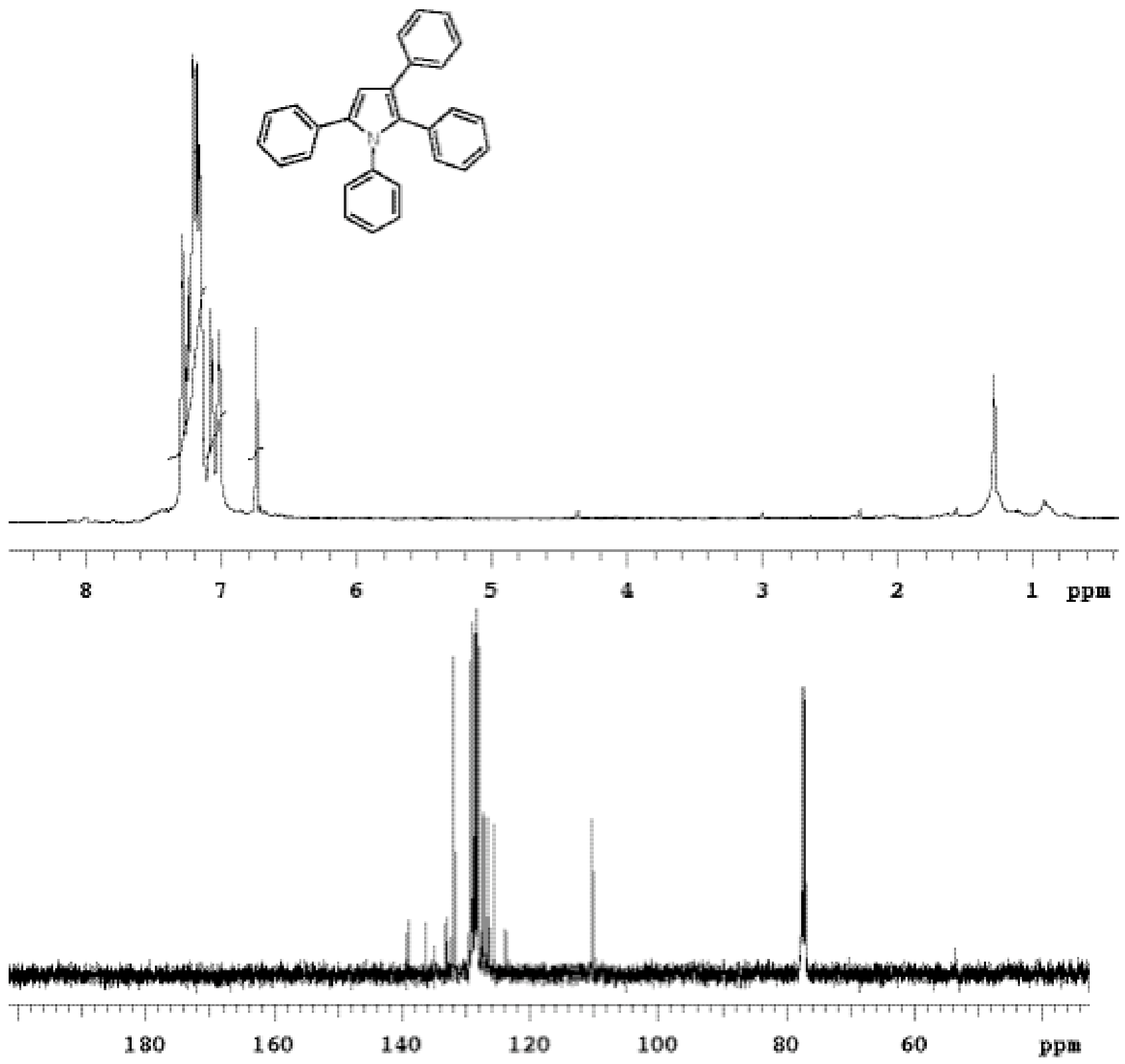
S62

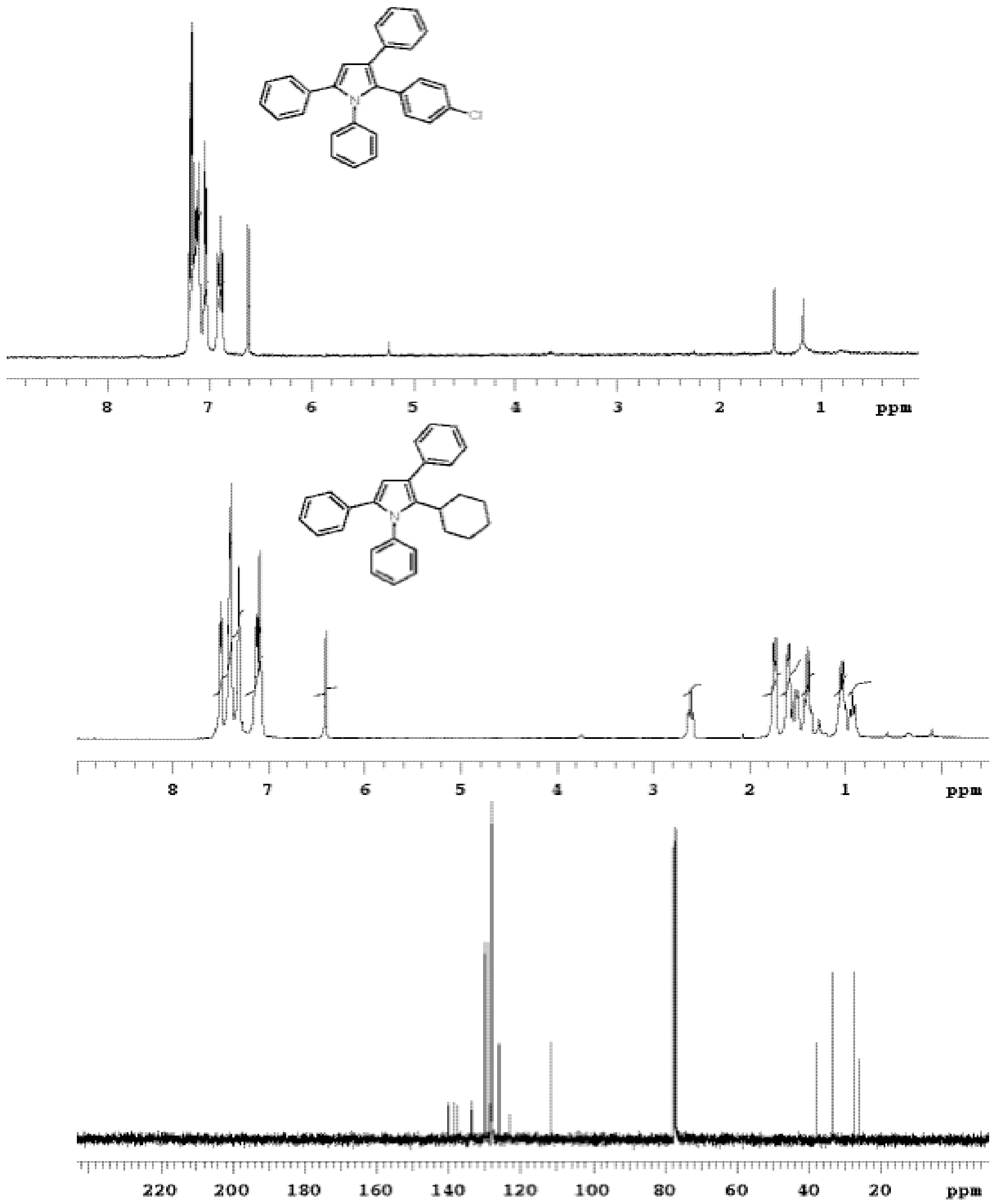




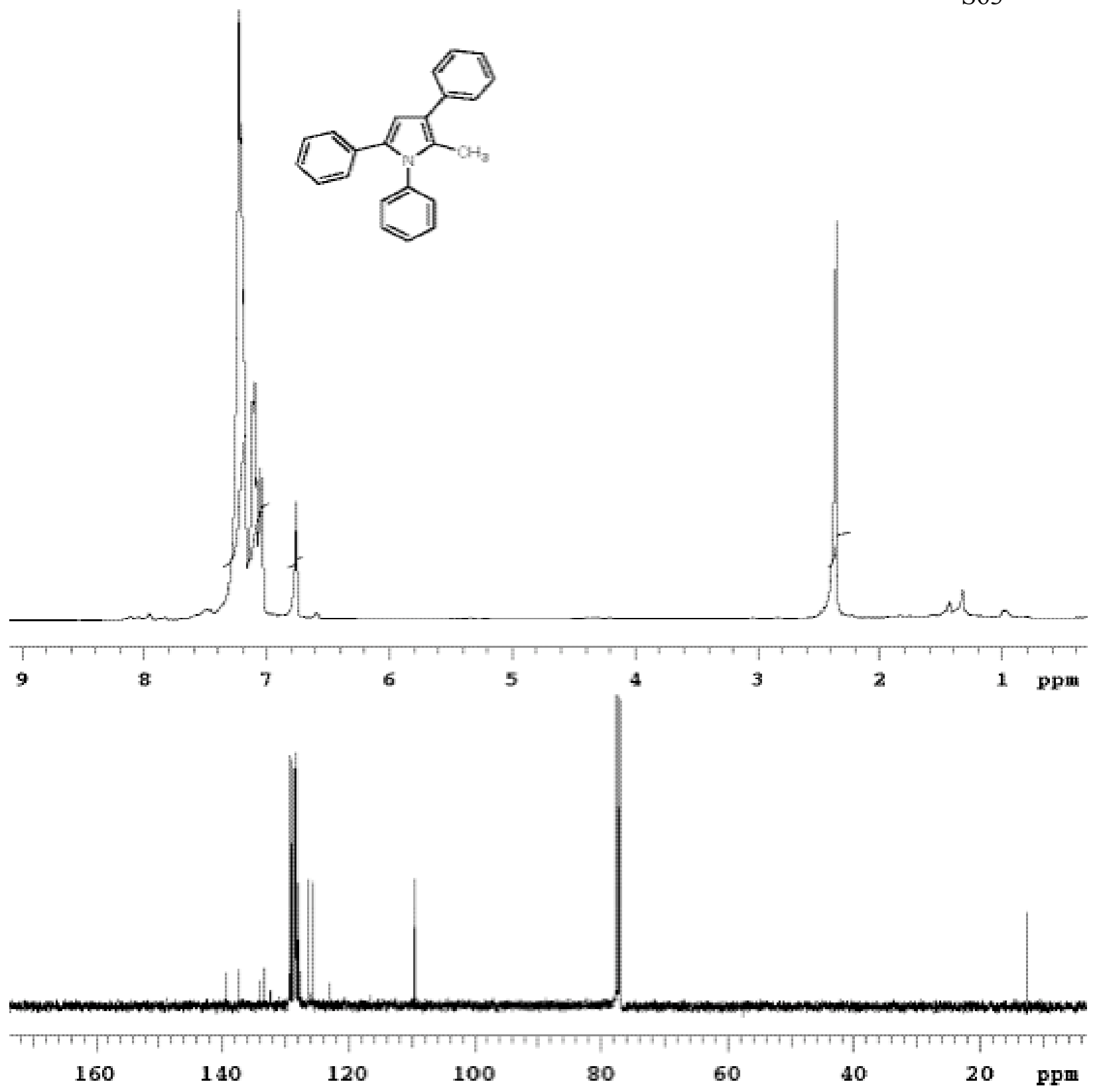




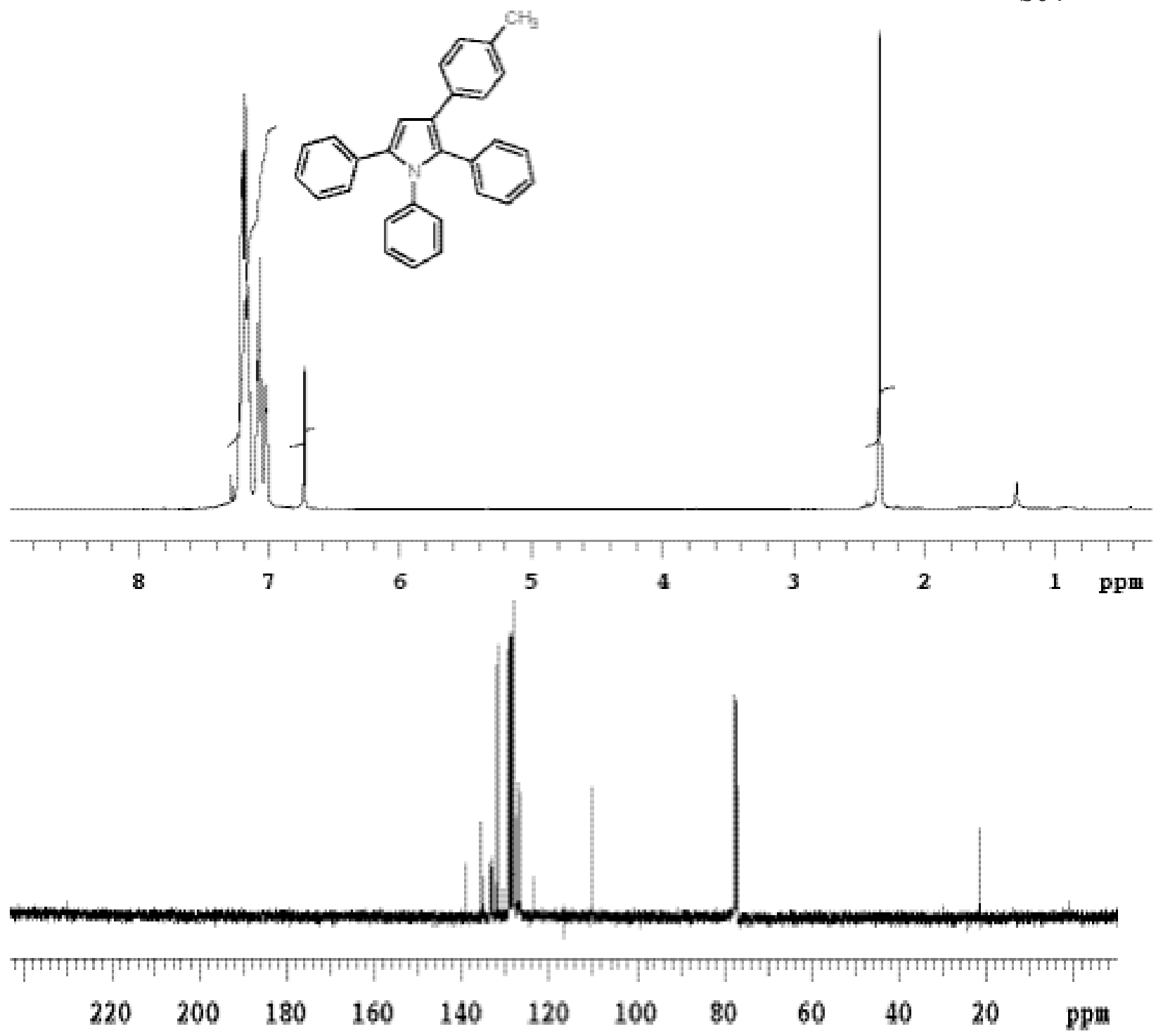


S65
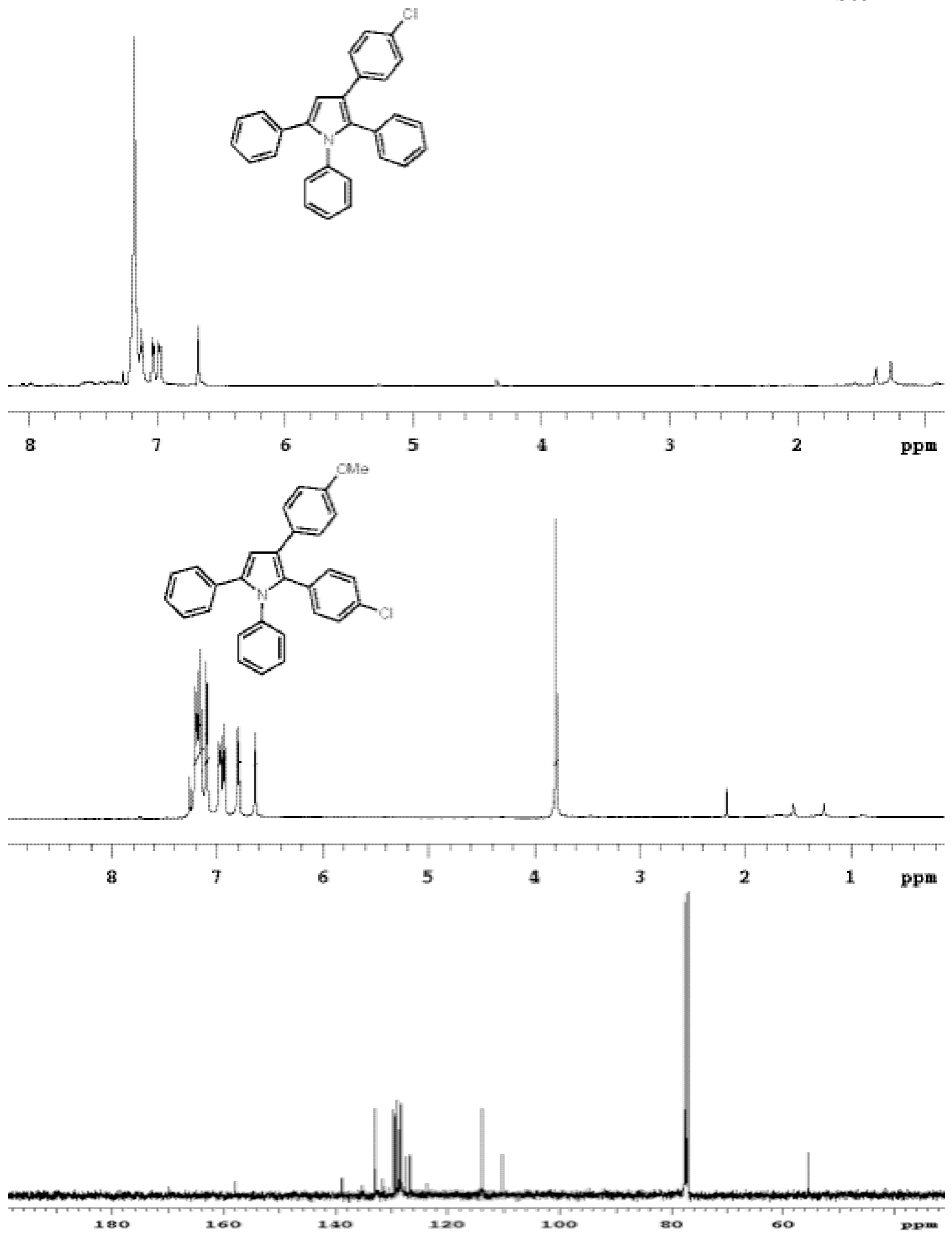
S66
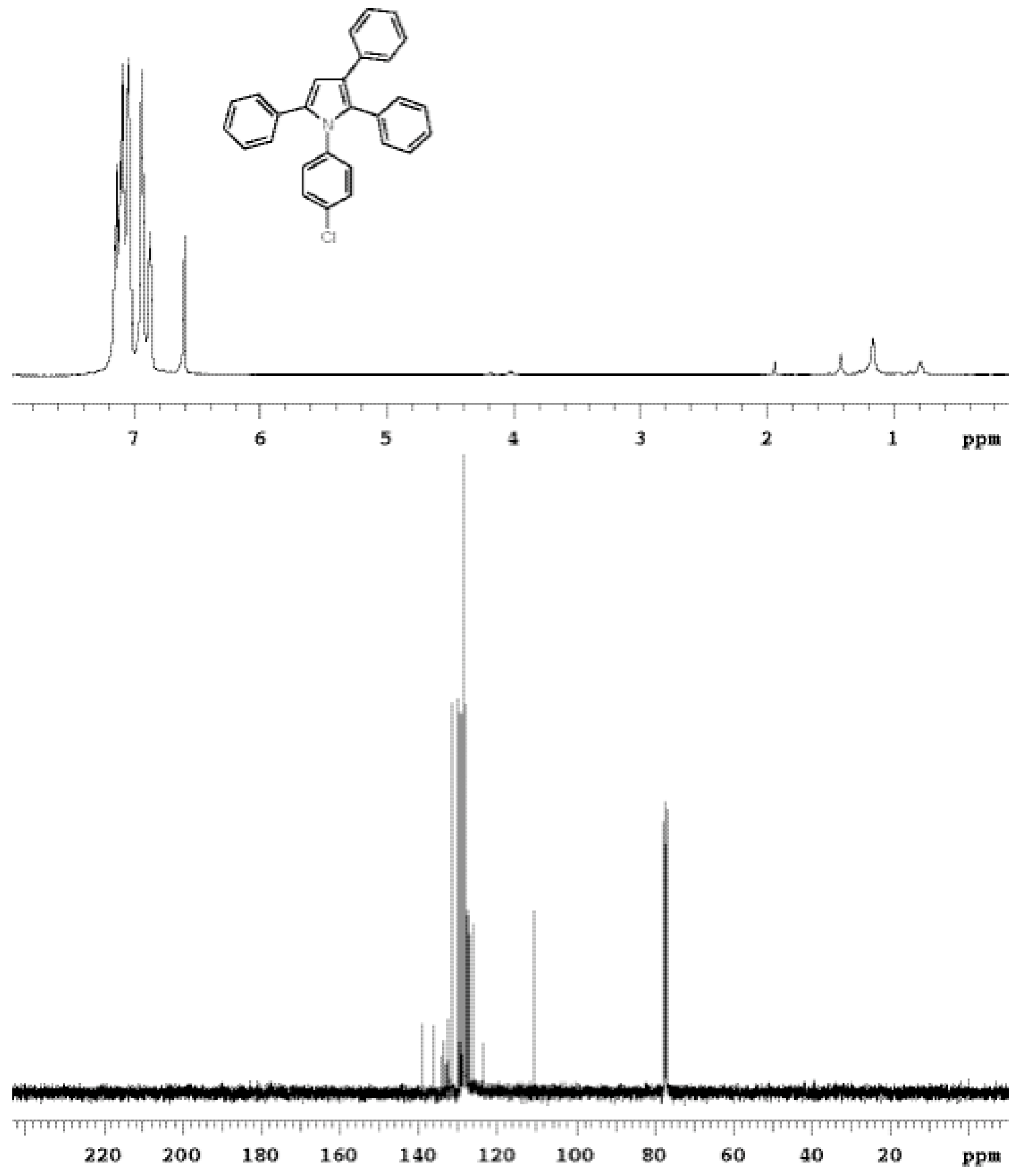


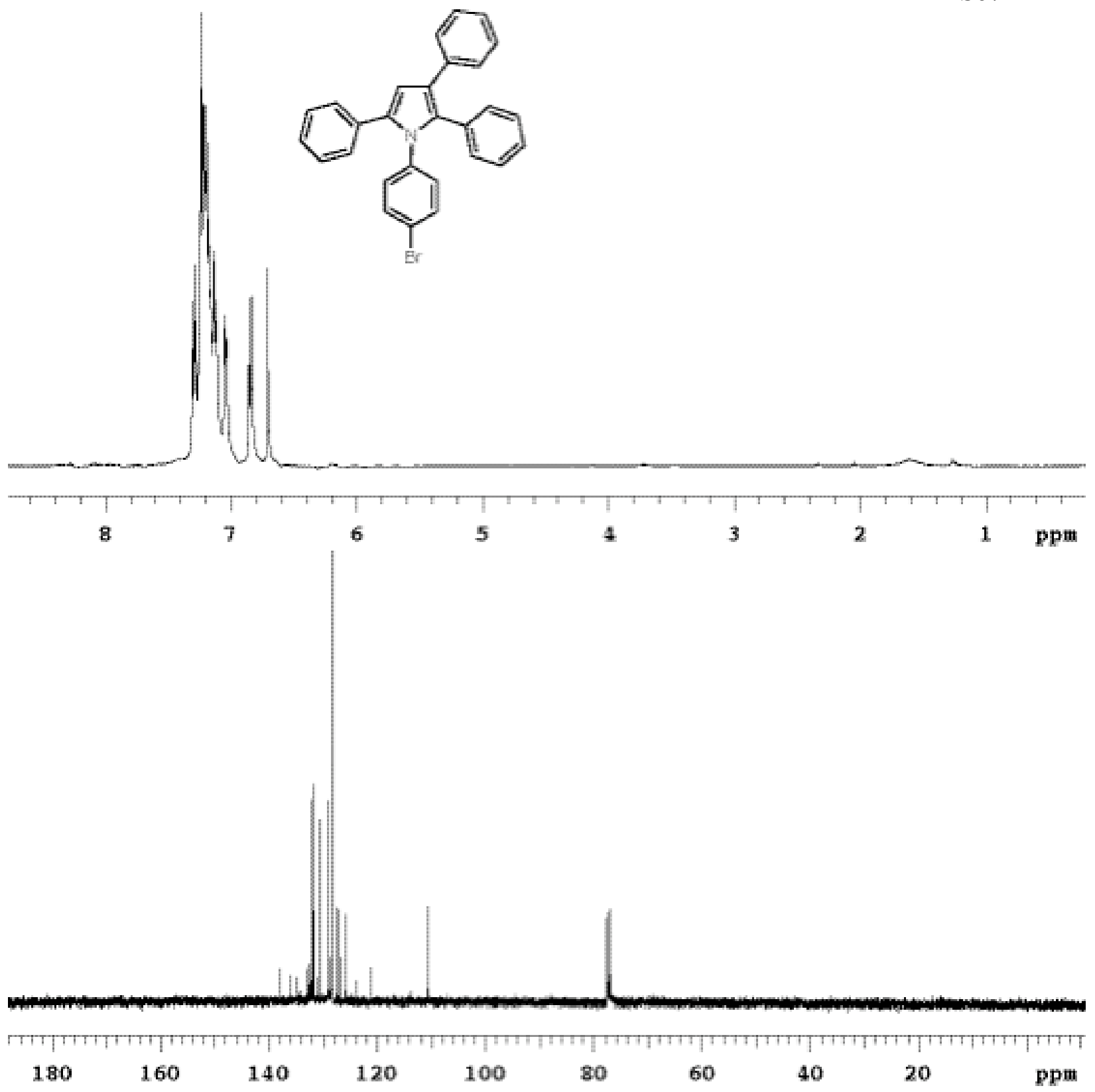


S68
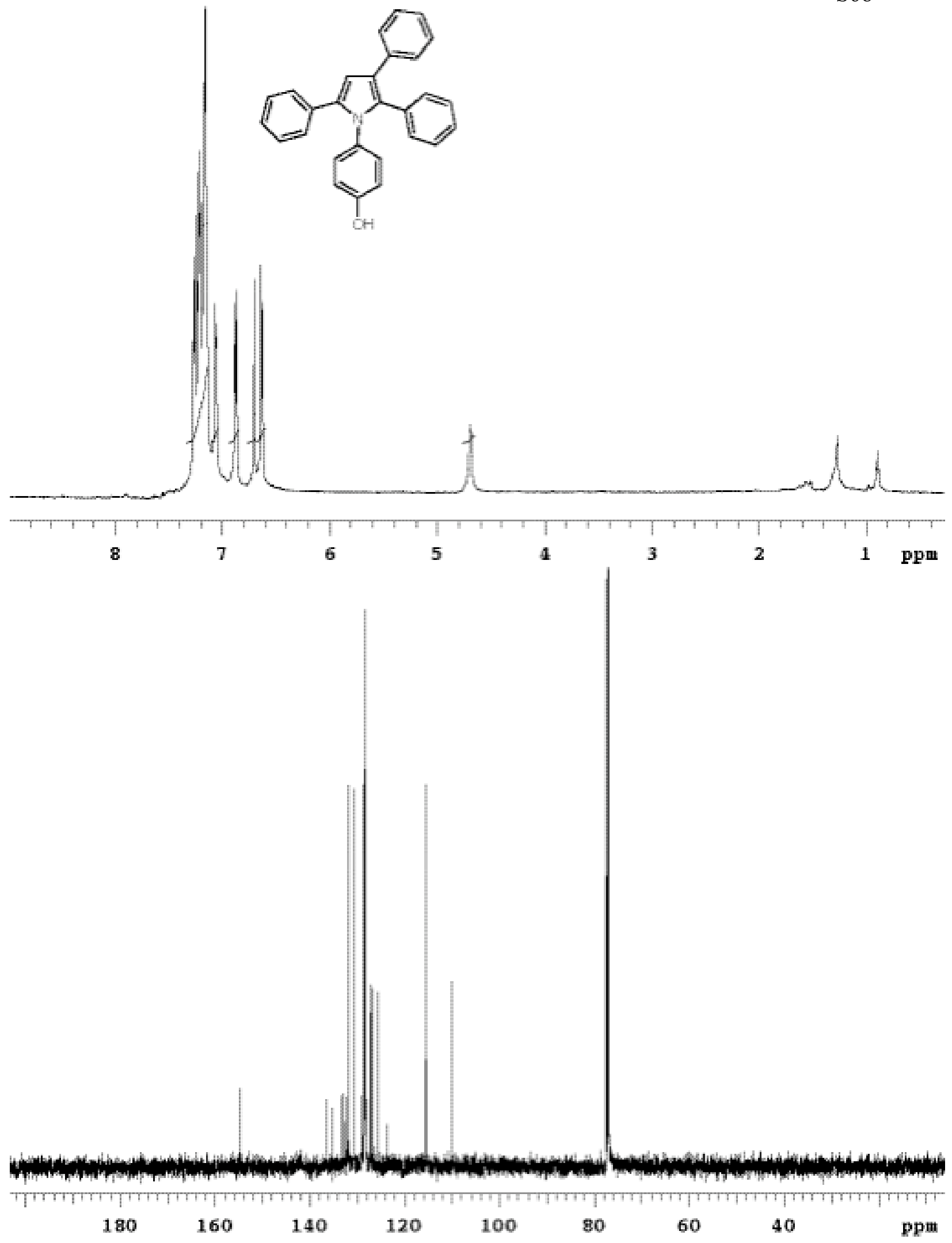
S69
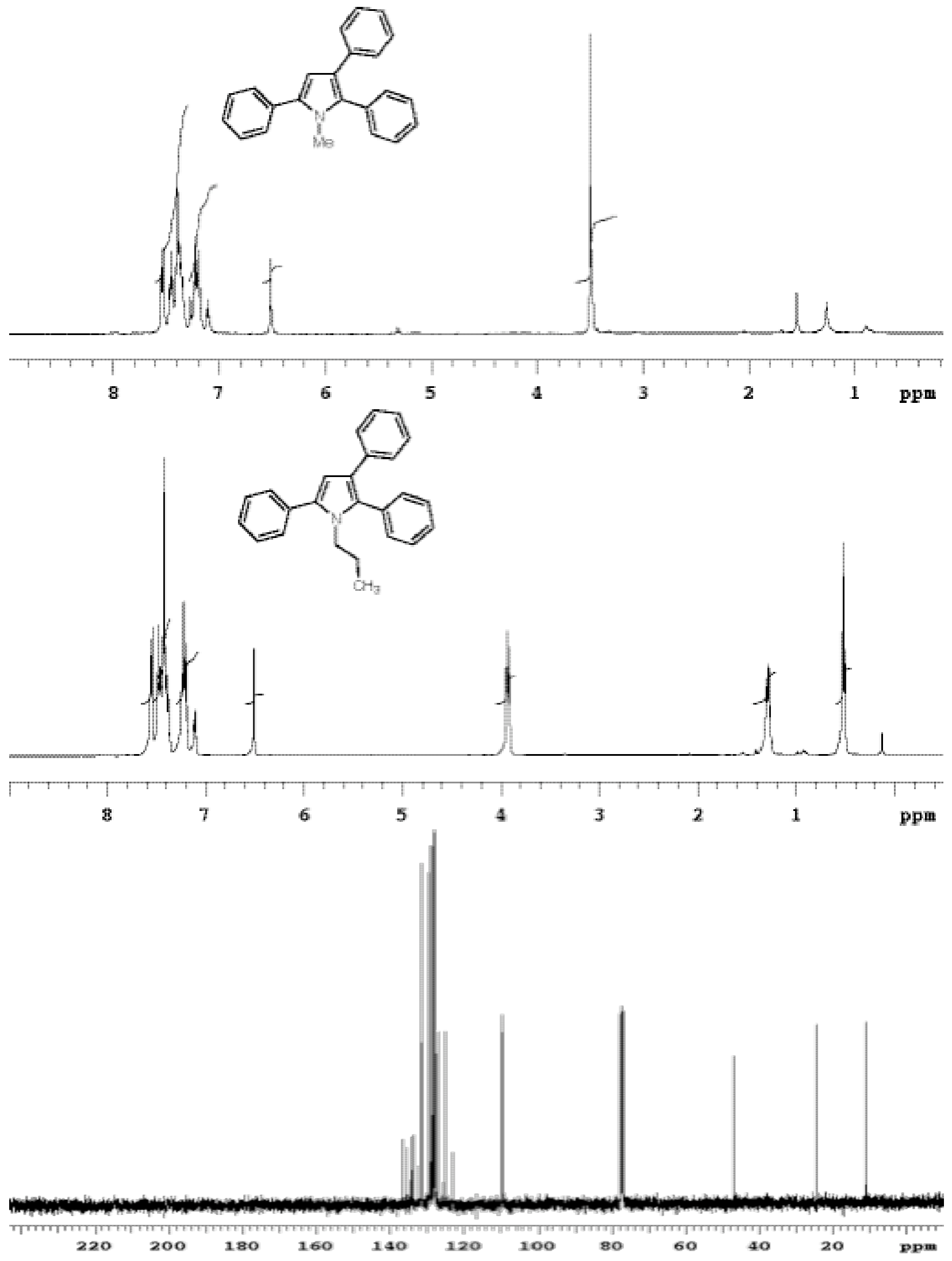
S70
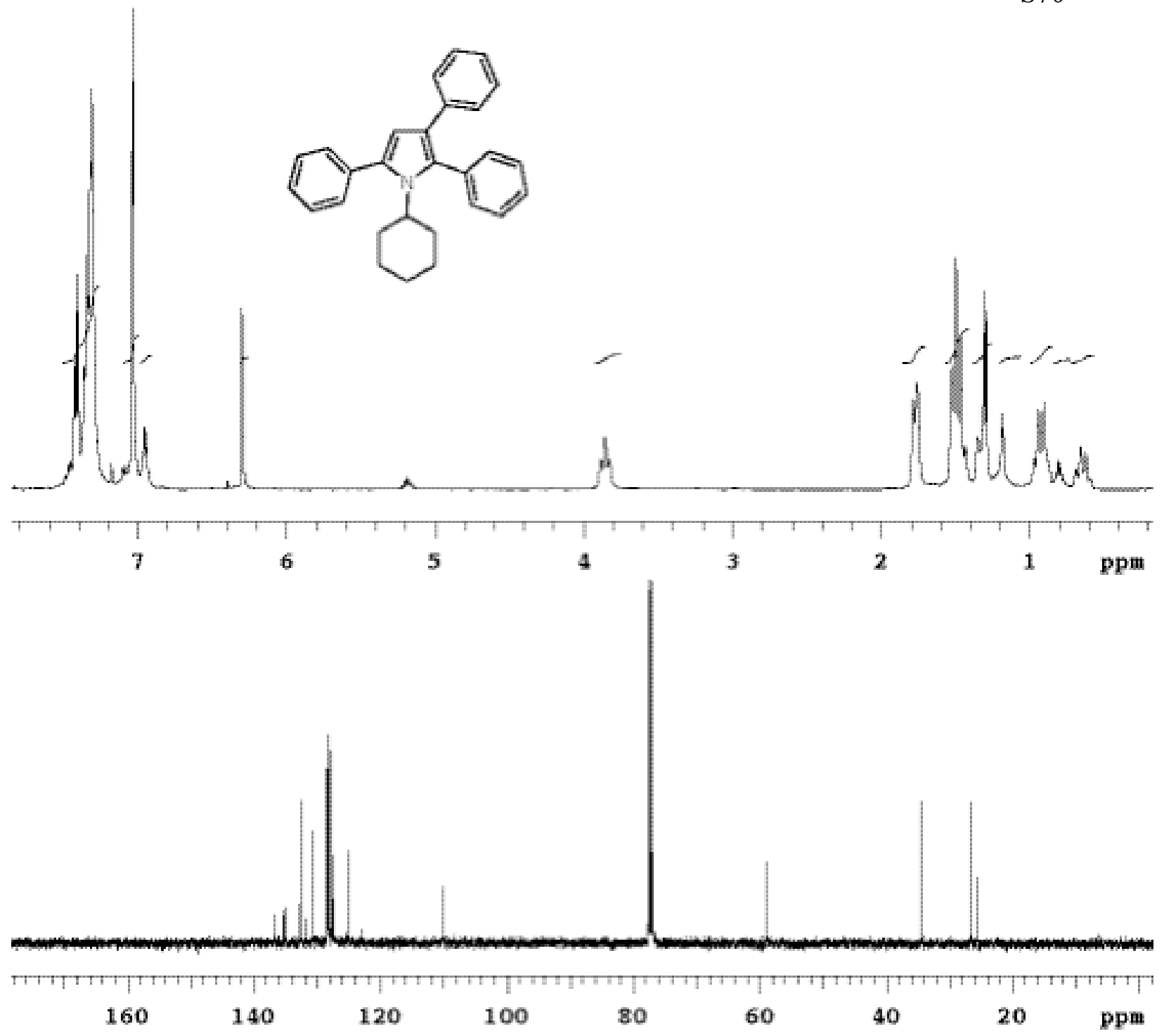


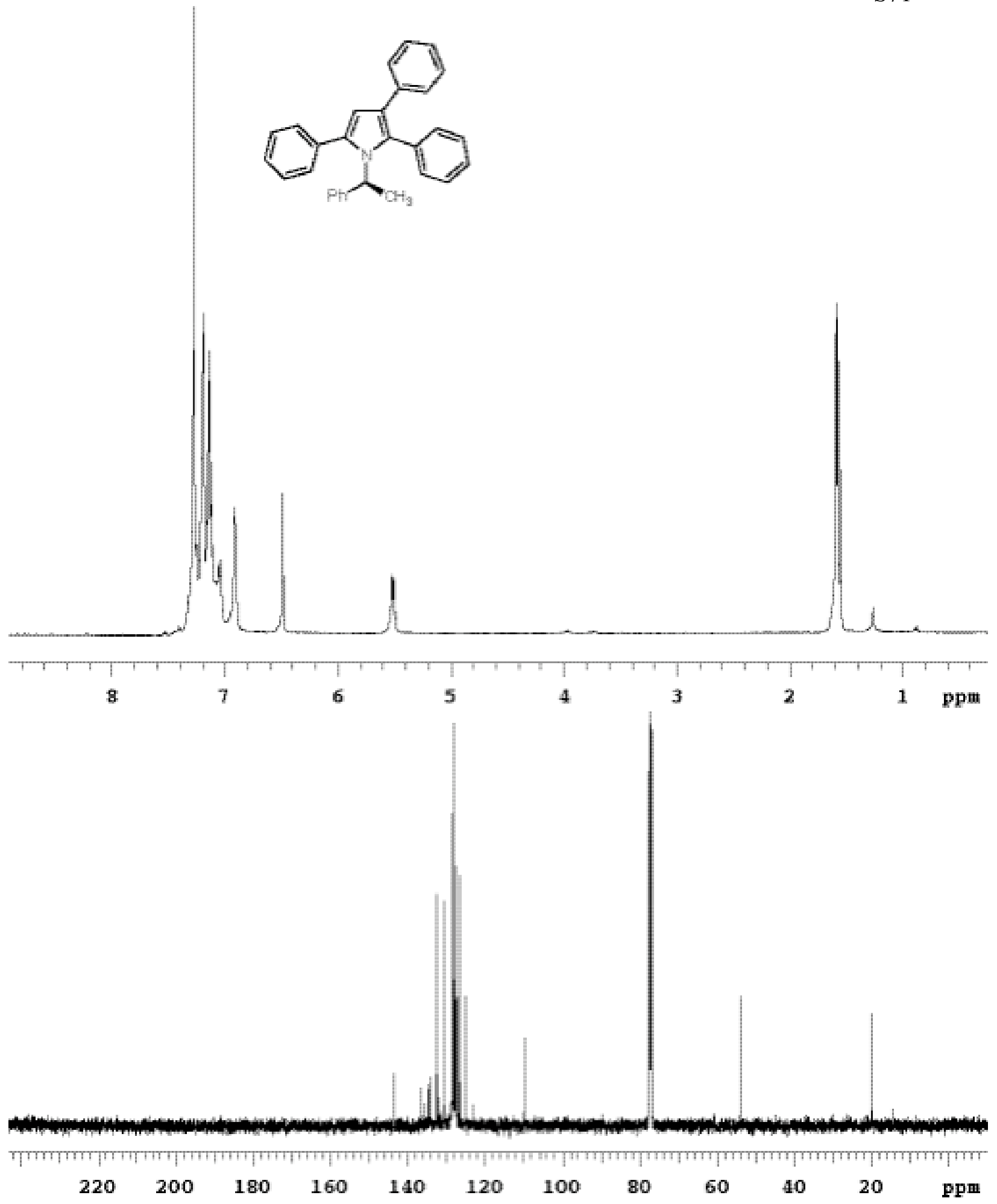



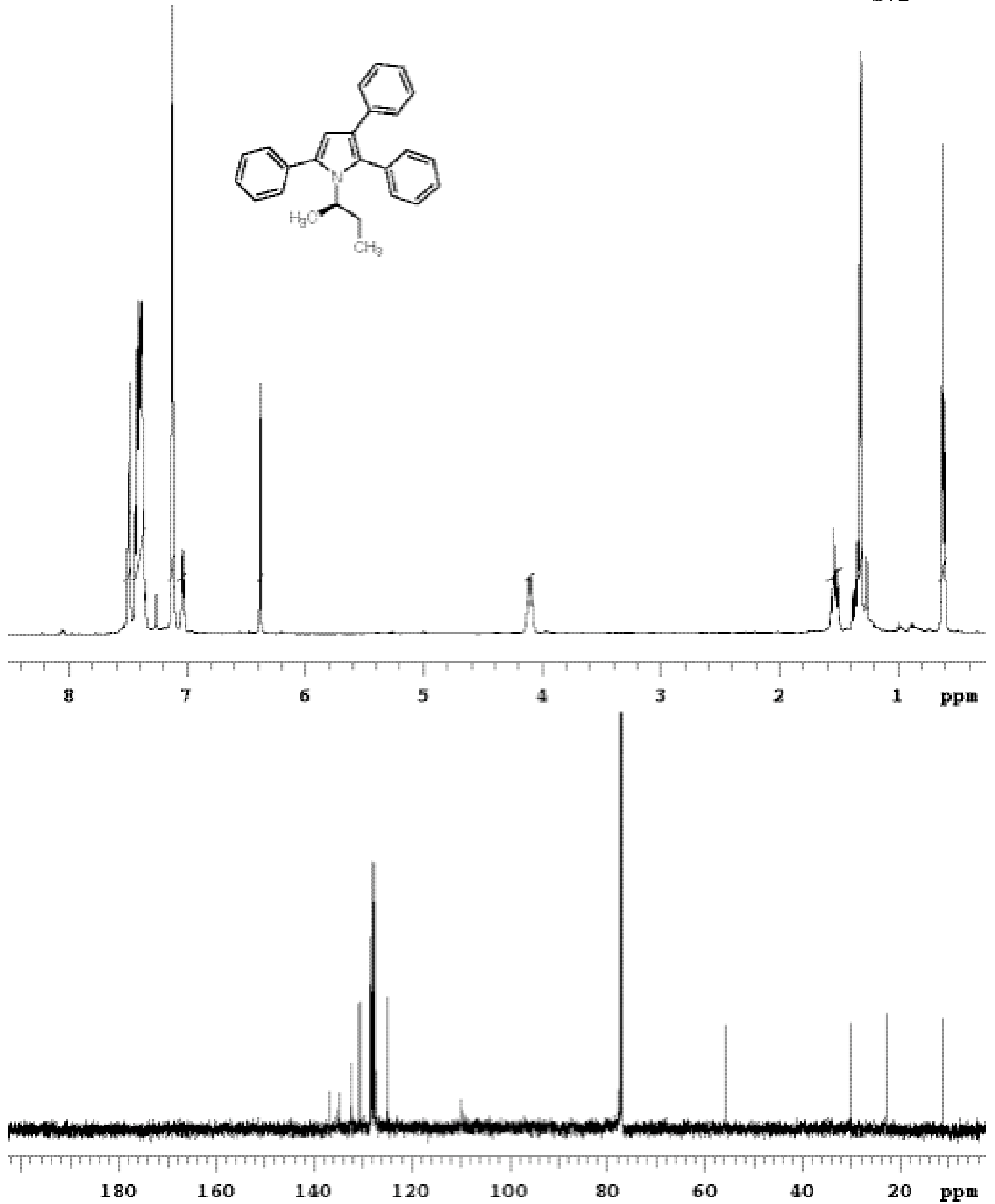
S73

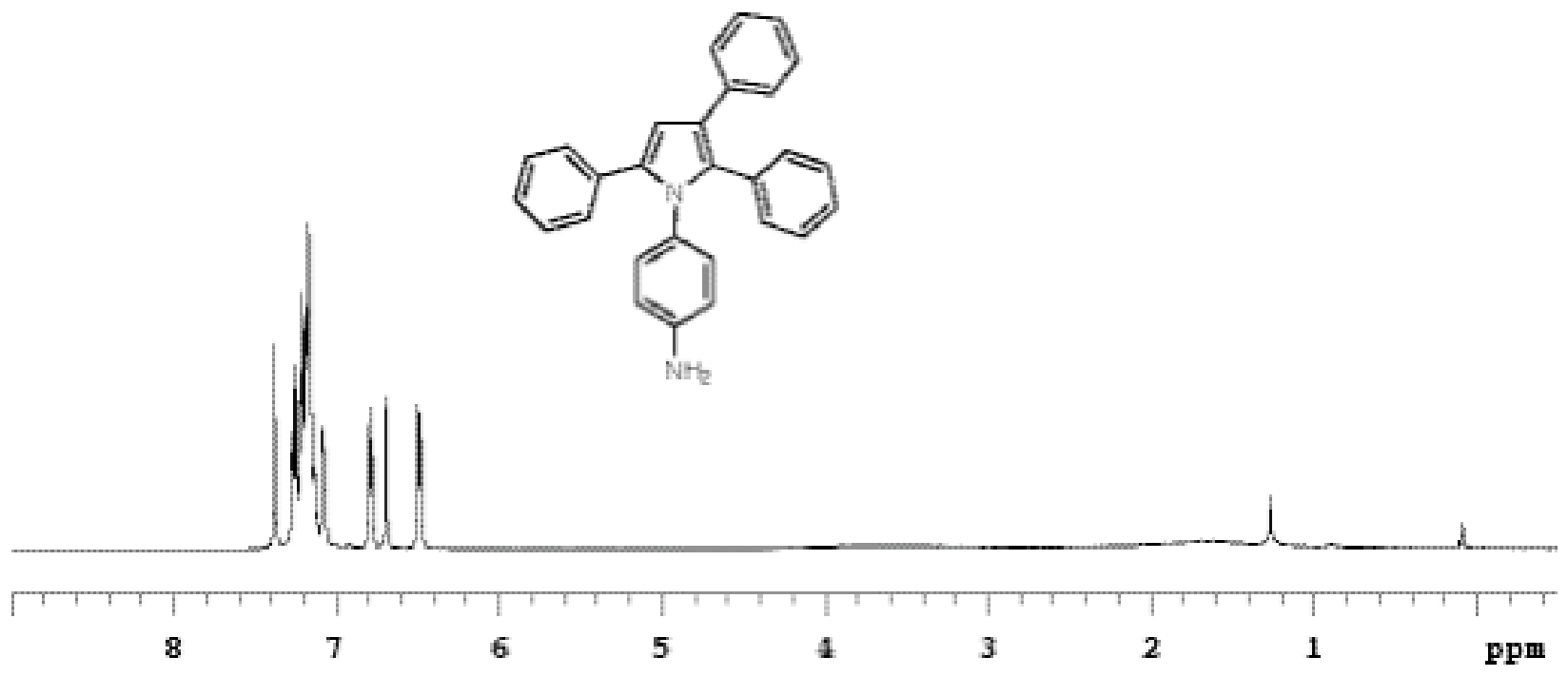



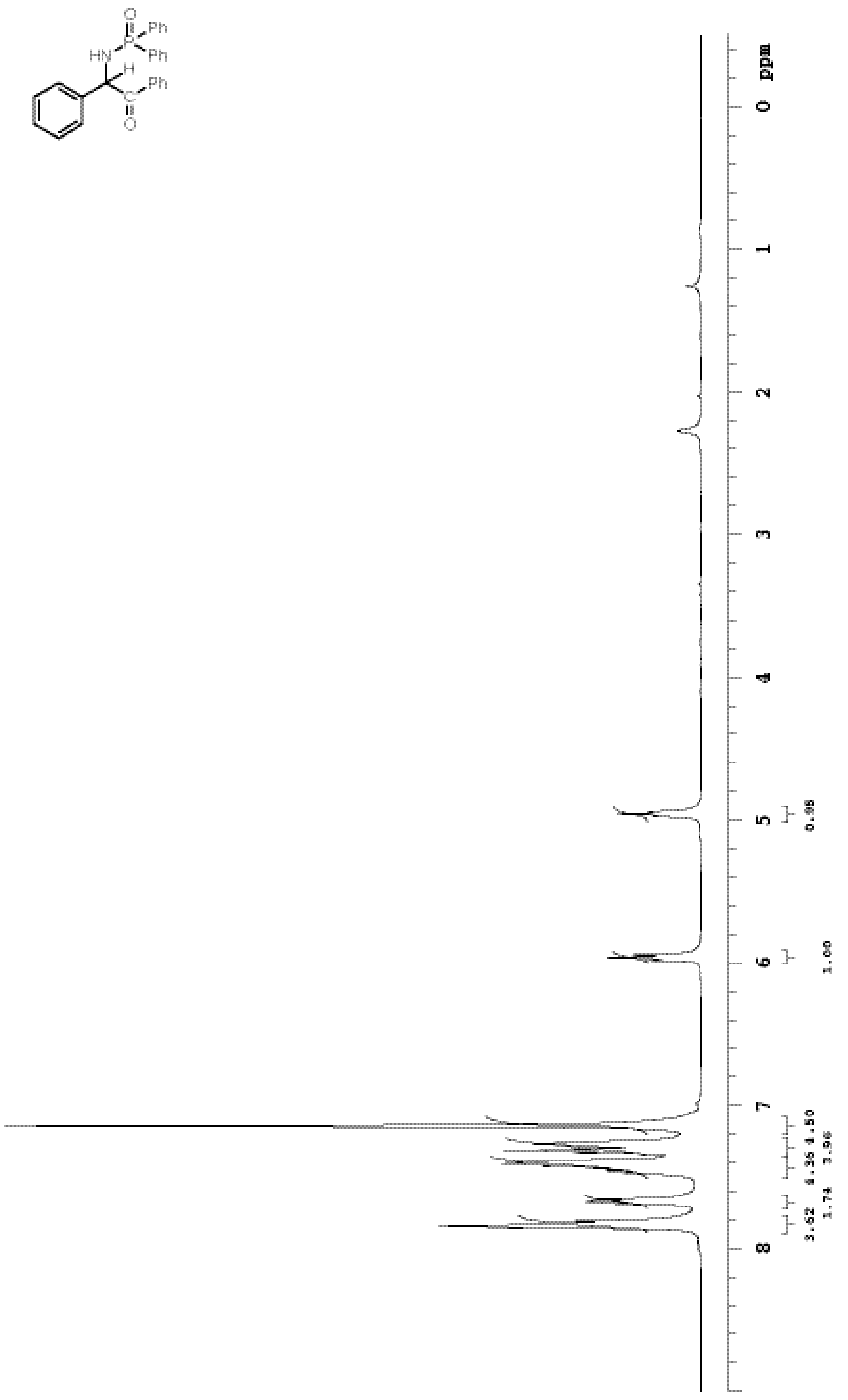
<smiles>O=C(P)C(NS(=O)(=O)c1ccccc1)(c1ccccc1)c1ccccc1</smiles>

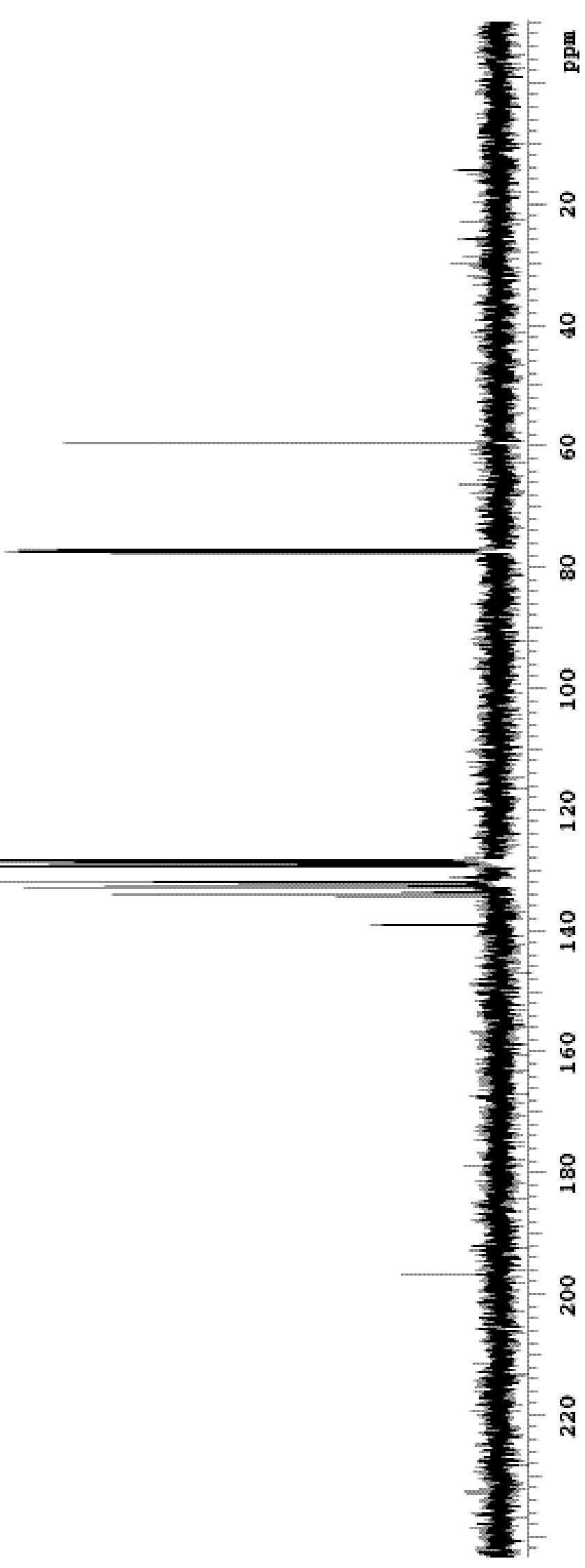



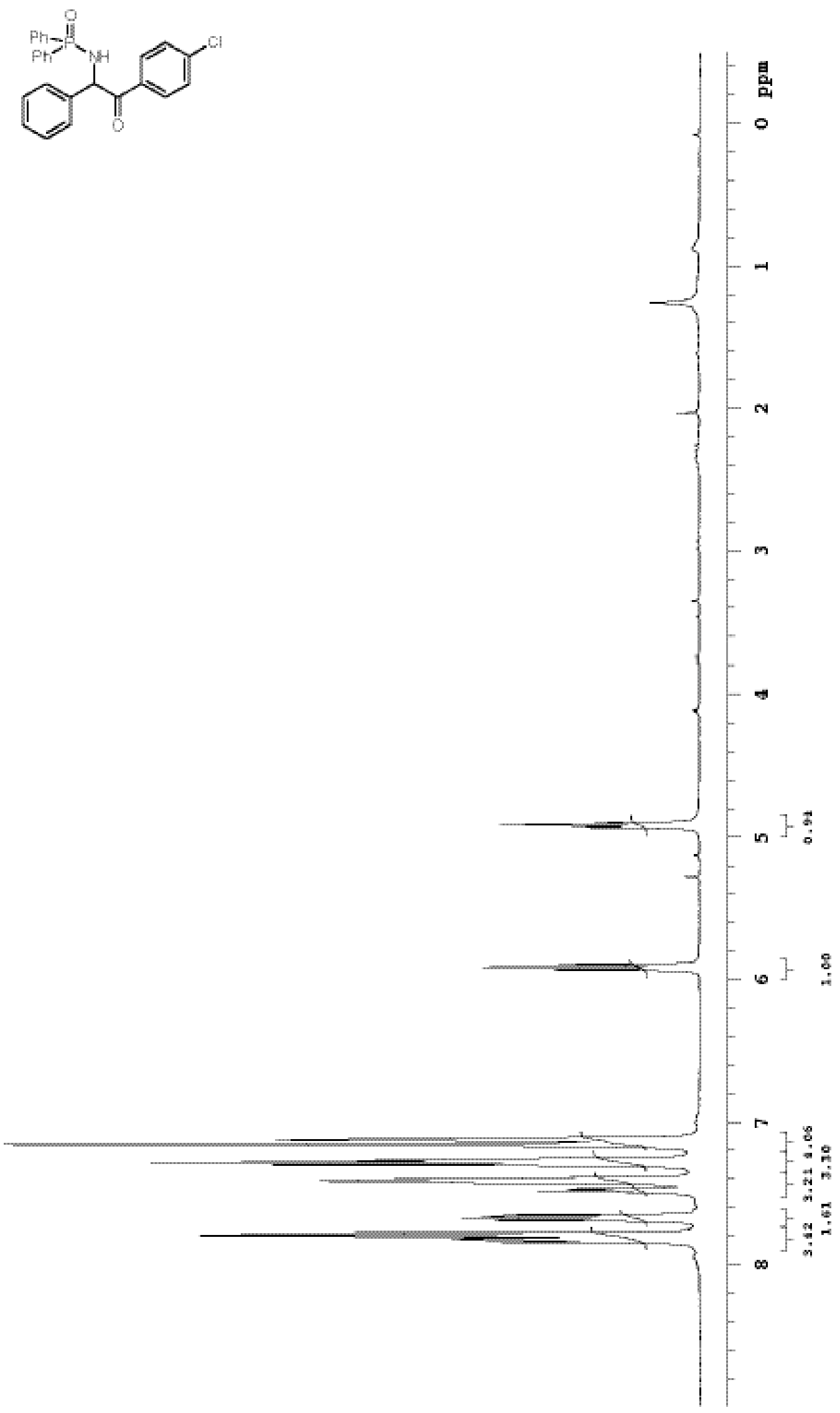
<smiles>O=C(c1ccc(Cl)cc1)C(NS(=O)(=O)c1ccccc1)c1ccccc1</smiles>

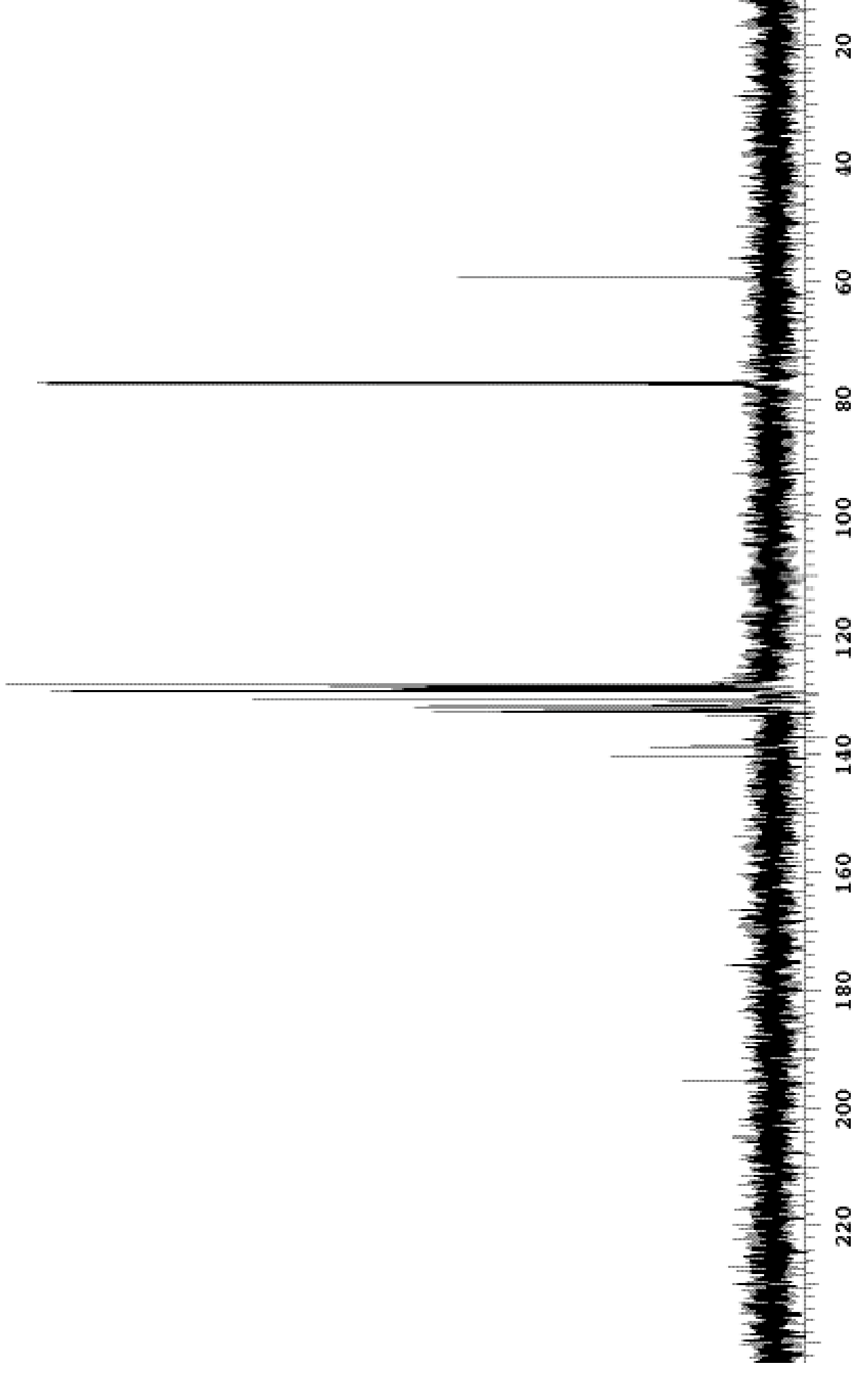


<smiles>CC(=O)C(NP(=O)(c1ccccc1)c1ccccc1)P(=O)(c1ccccc1)c1ccccc1</smiles>

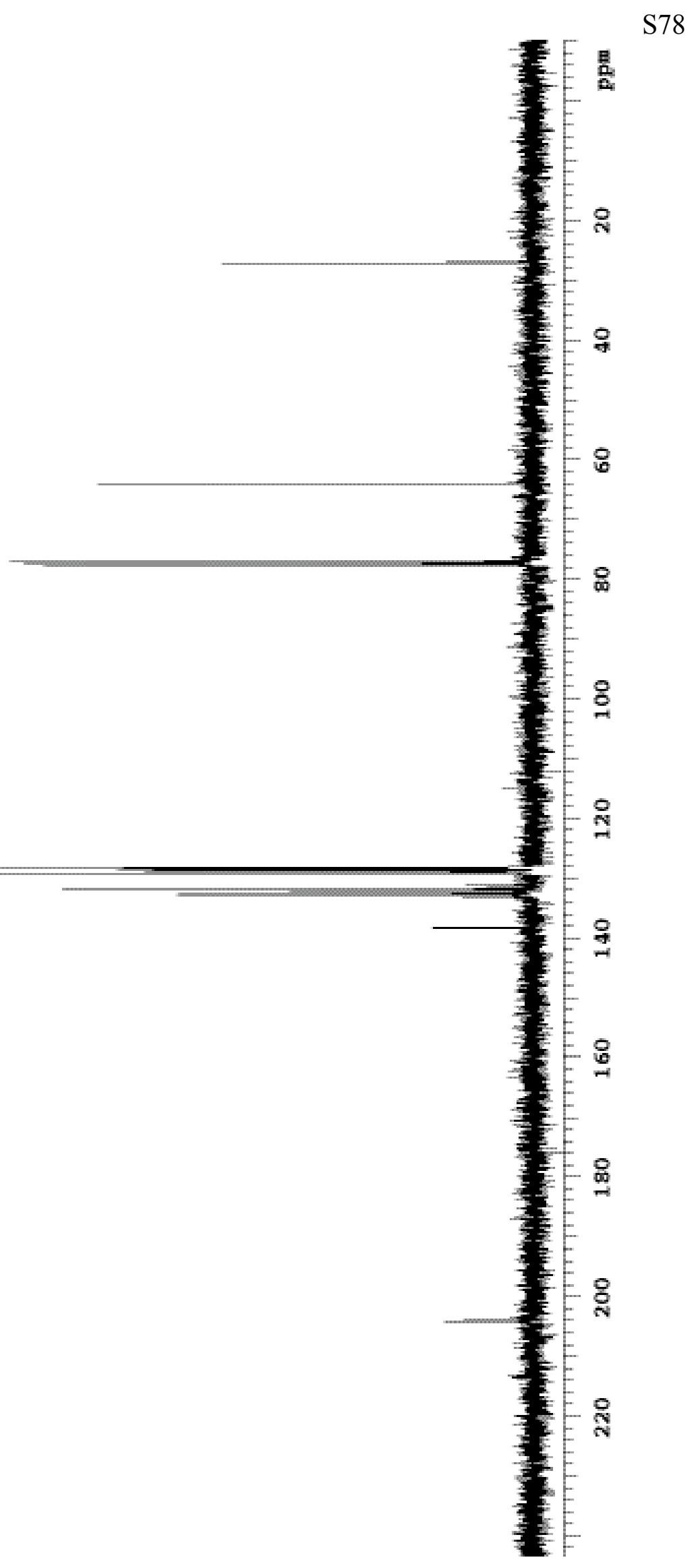



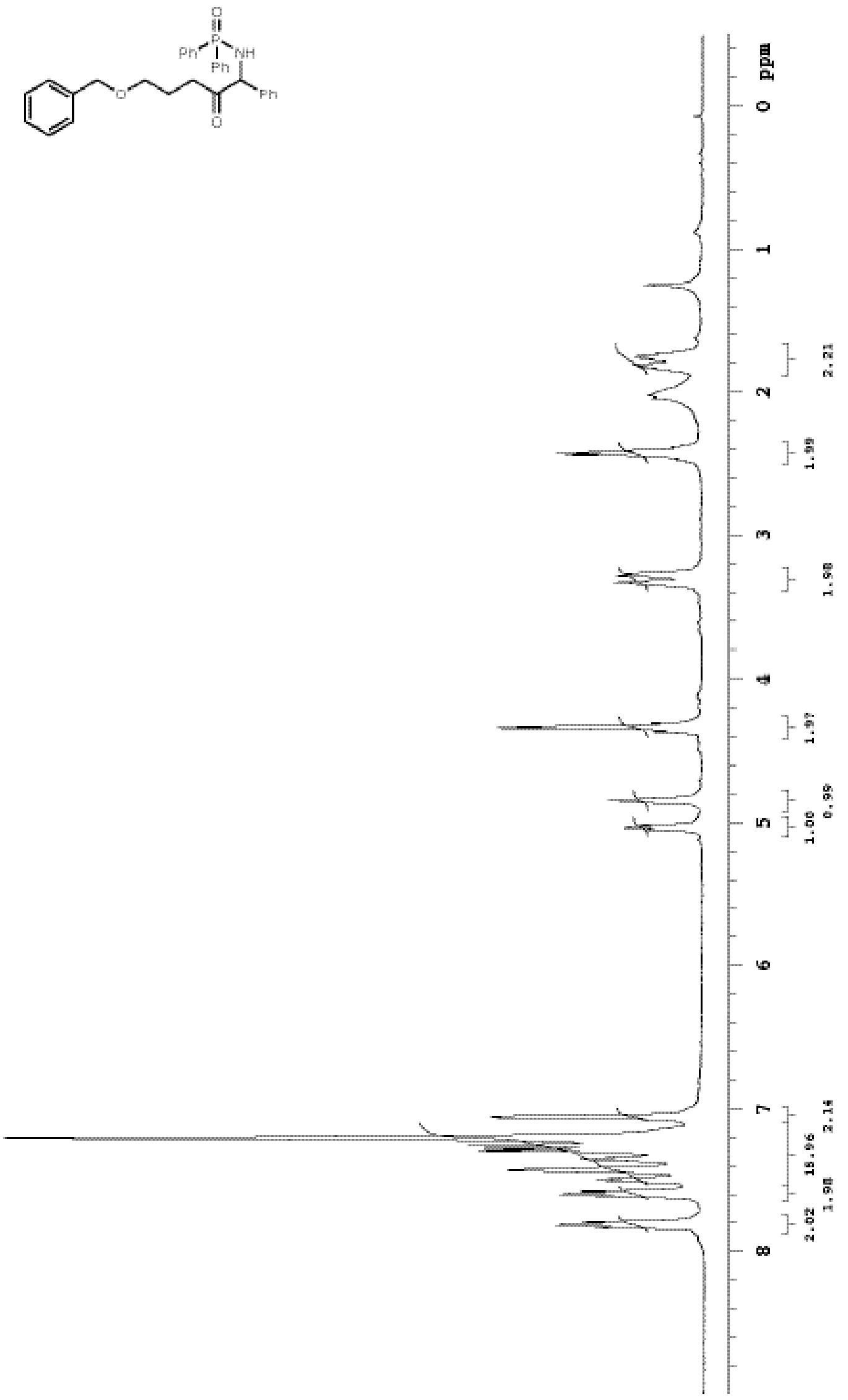


$$
4
$$



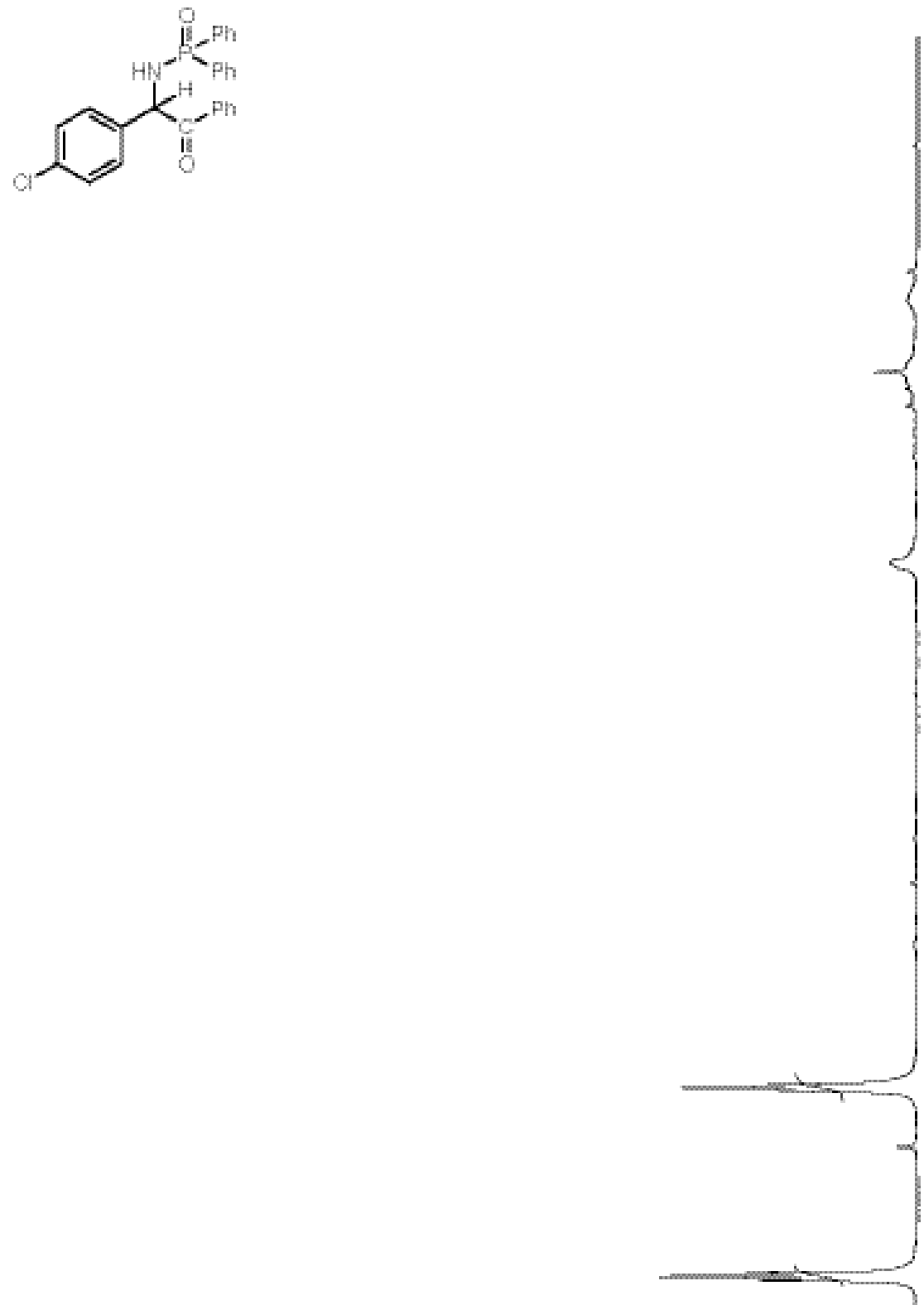

- 目

。

- 0
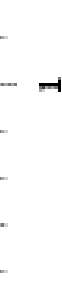

-
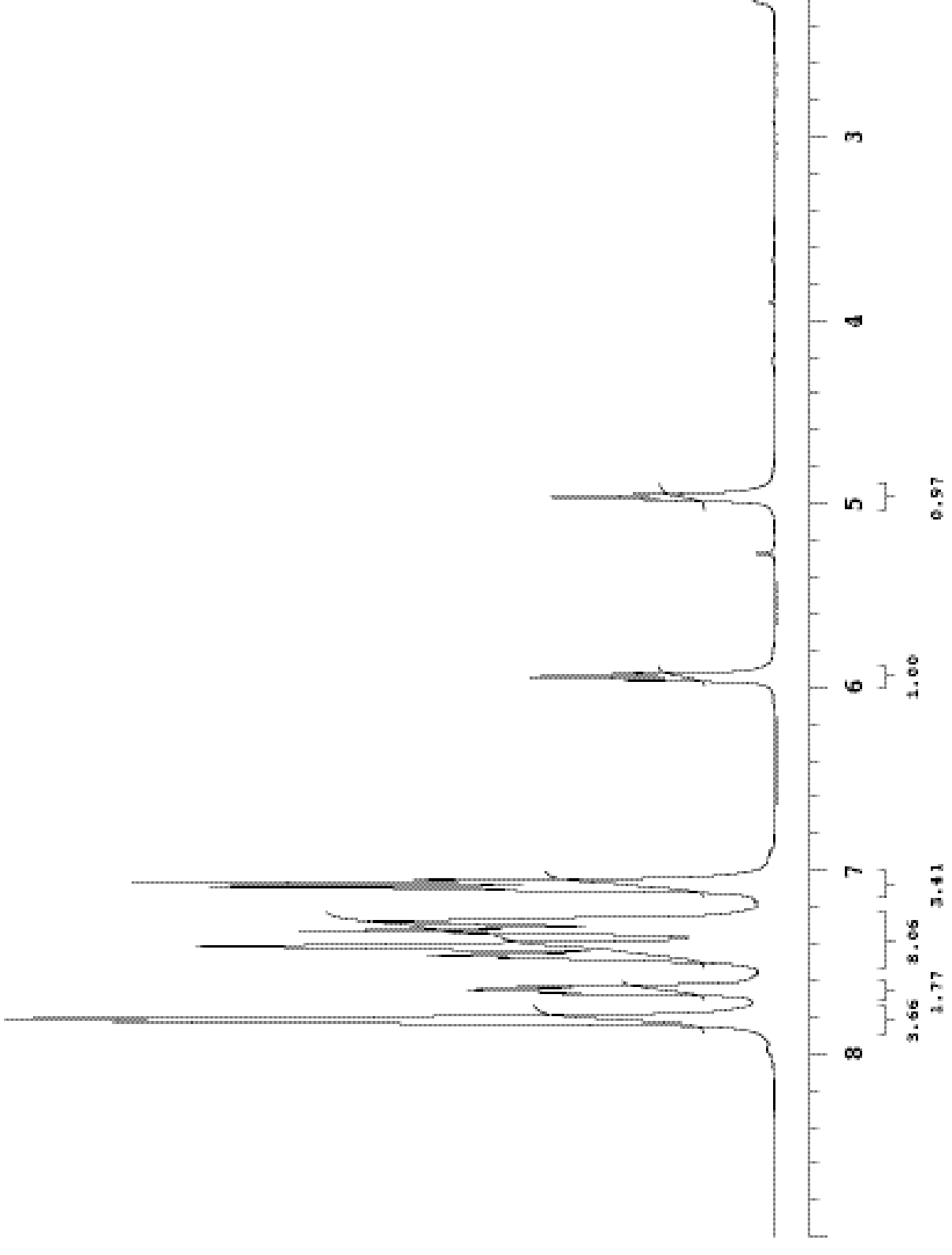

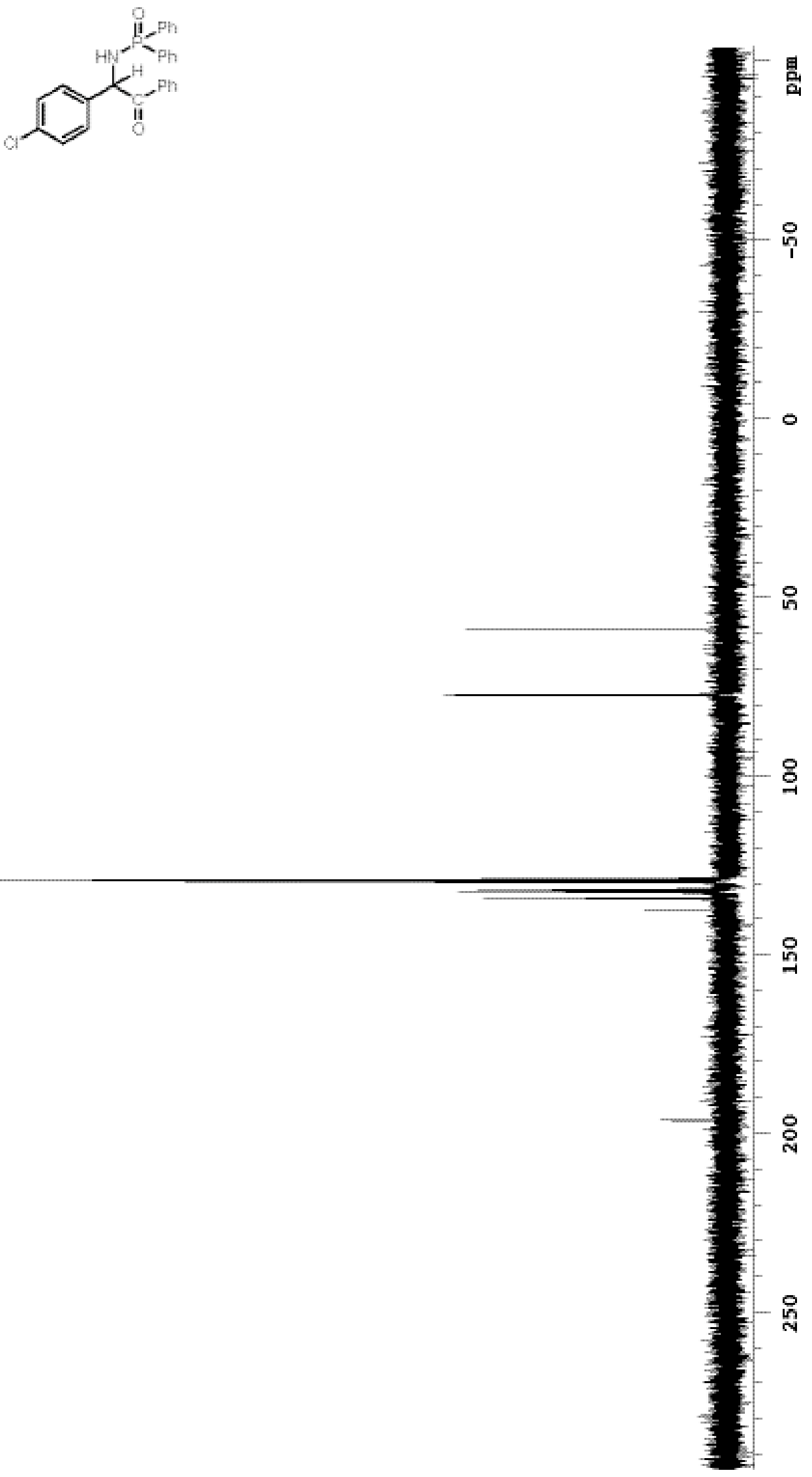

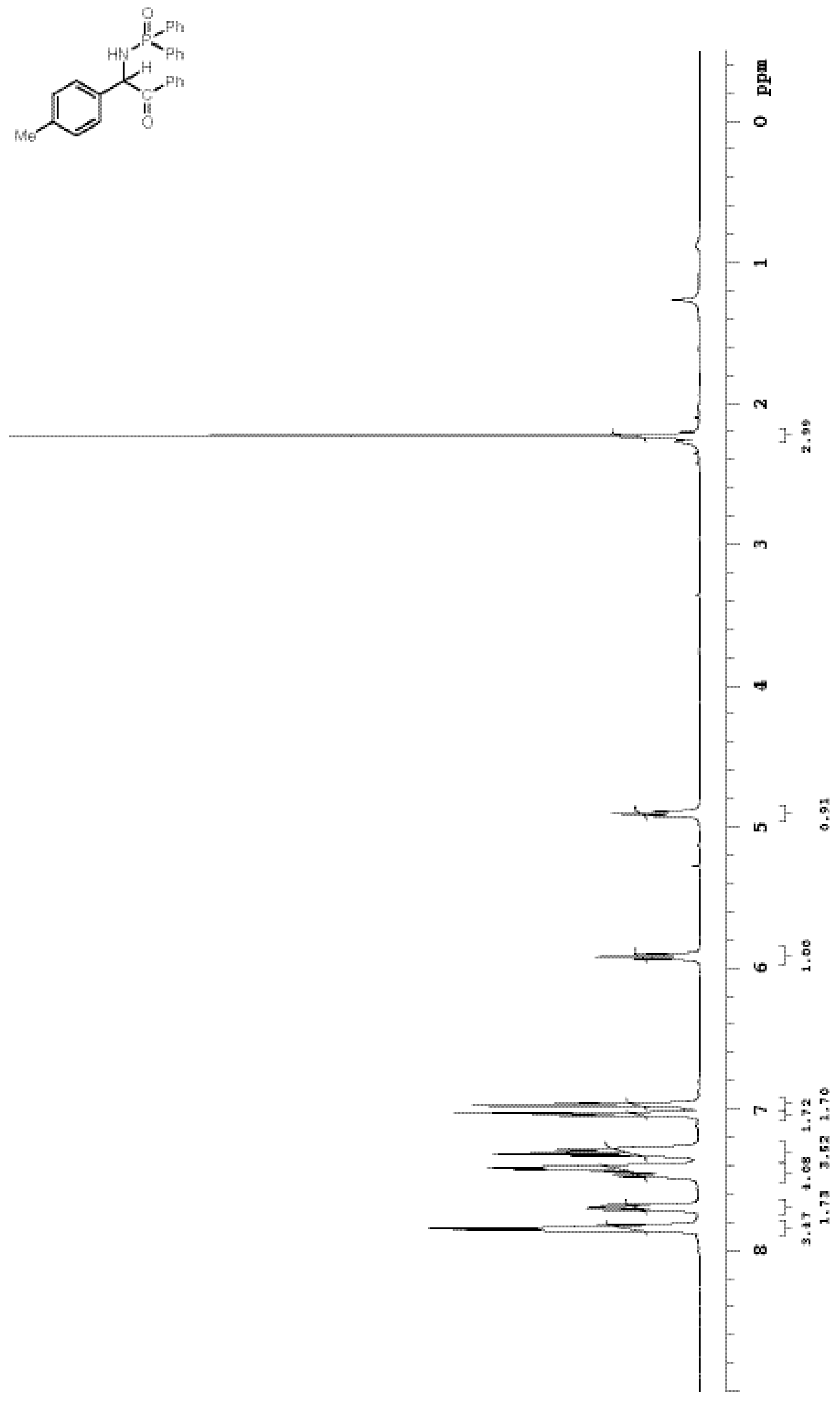

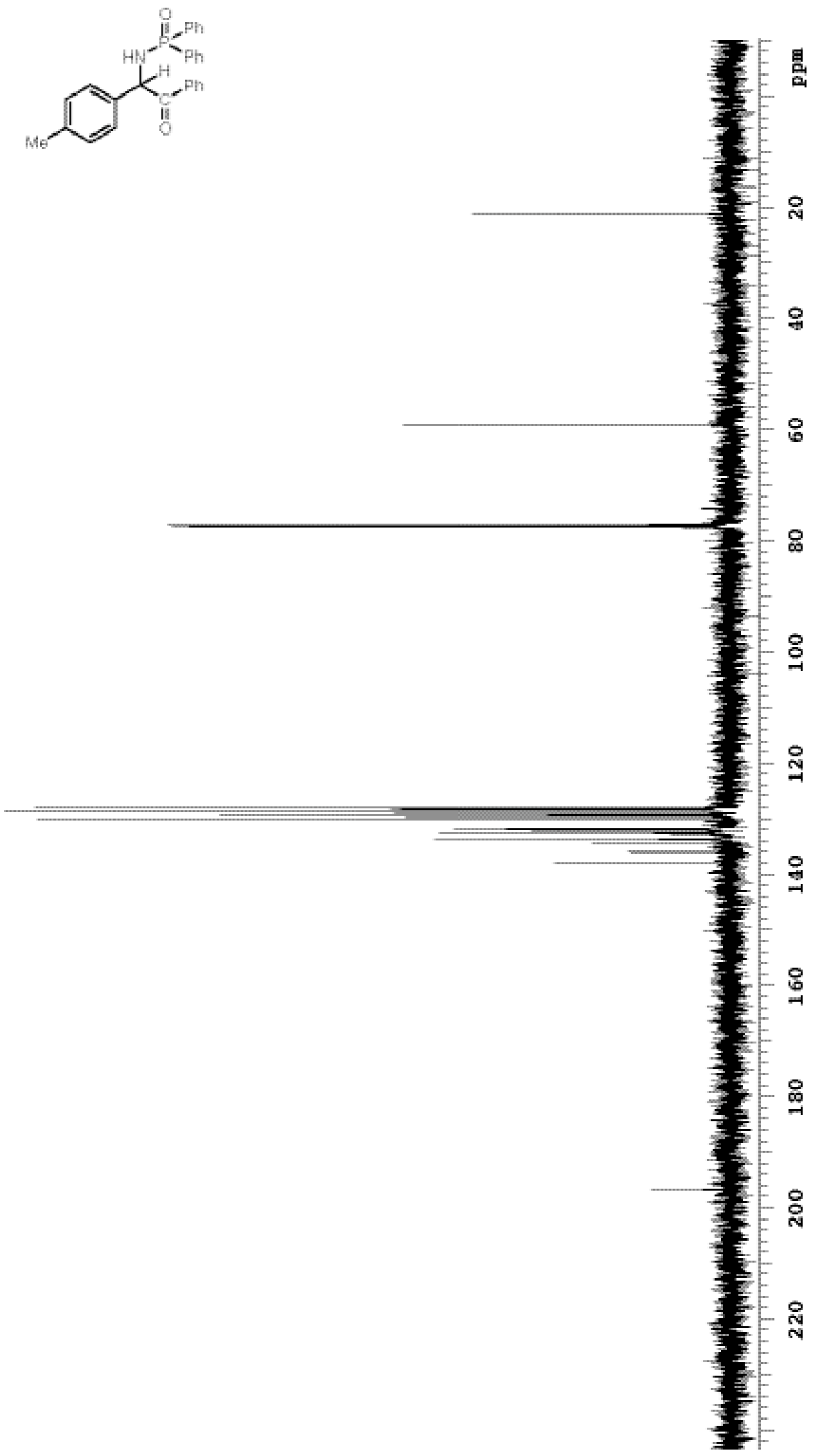

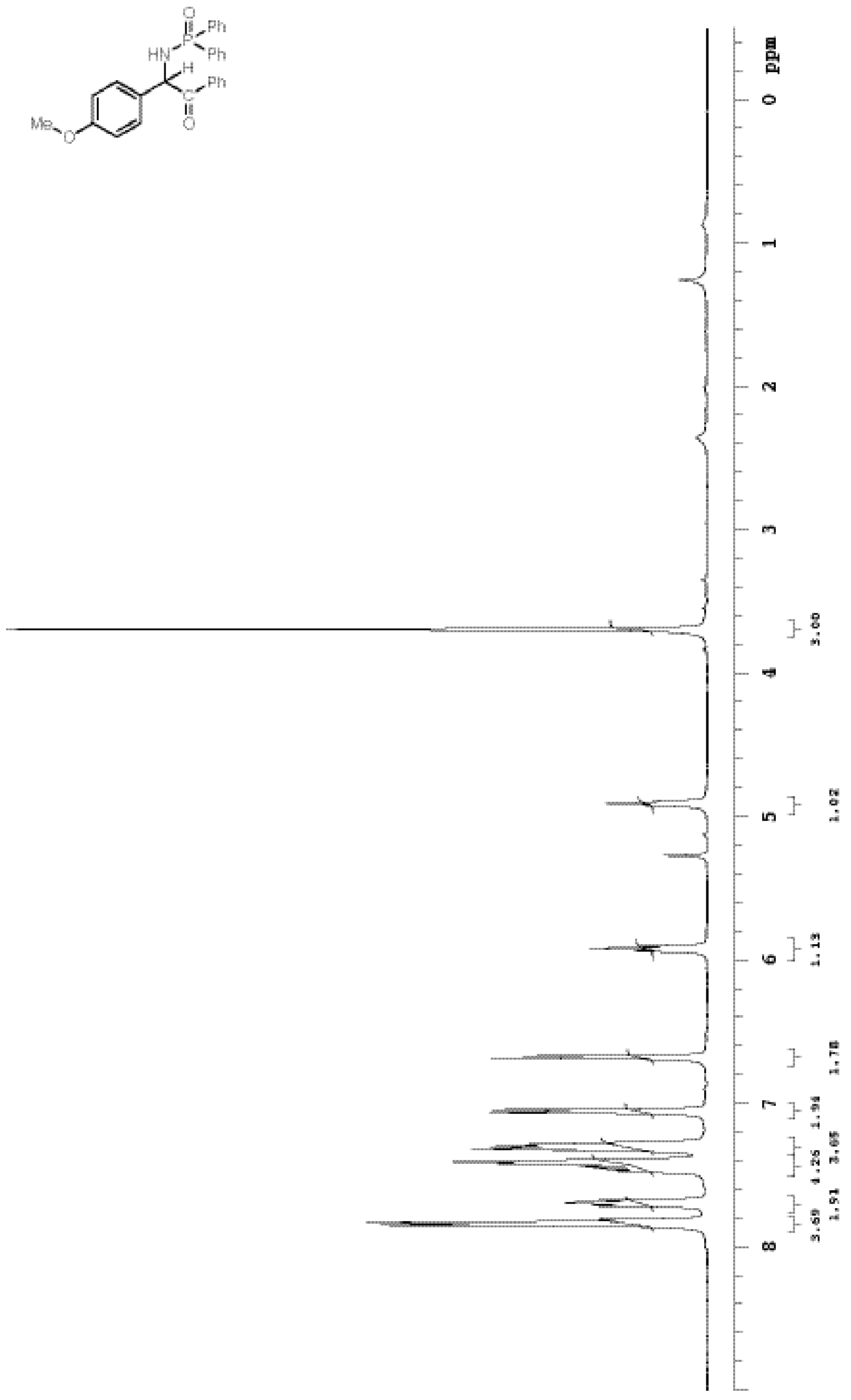

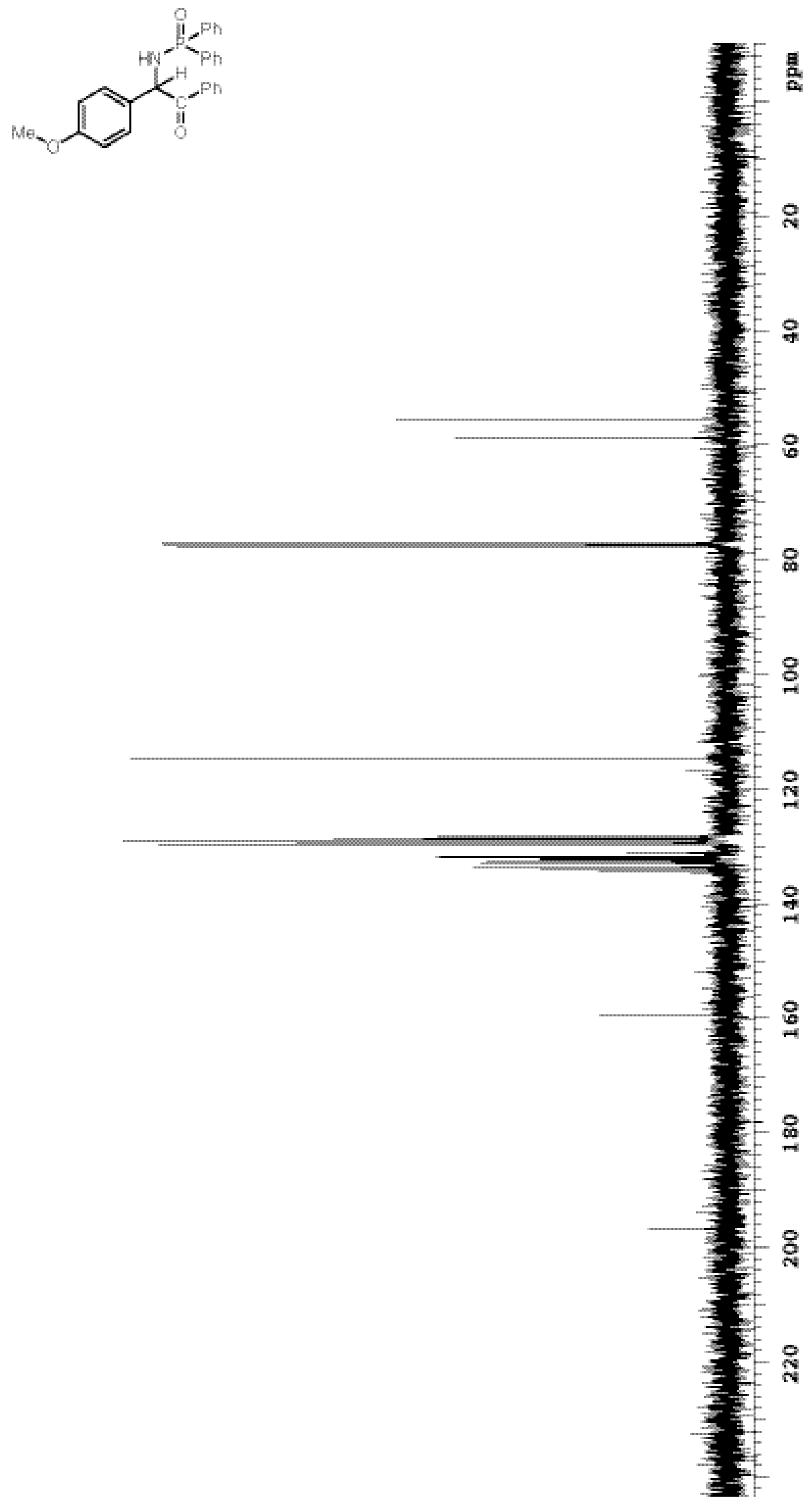

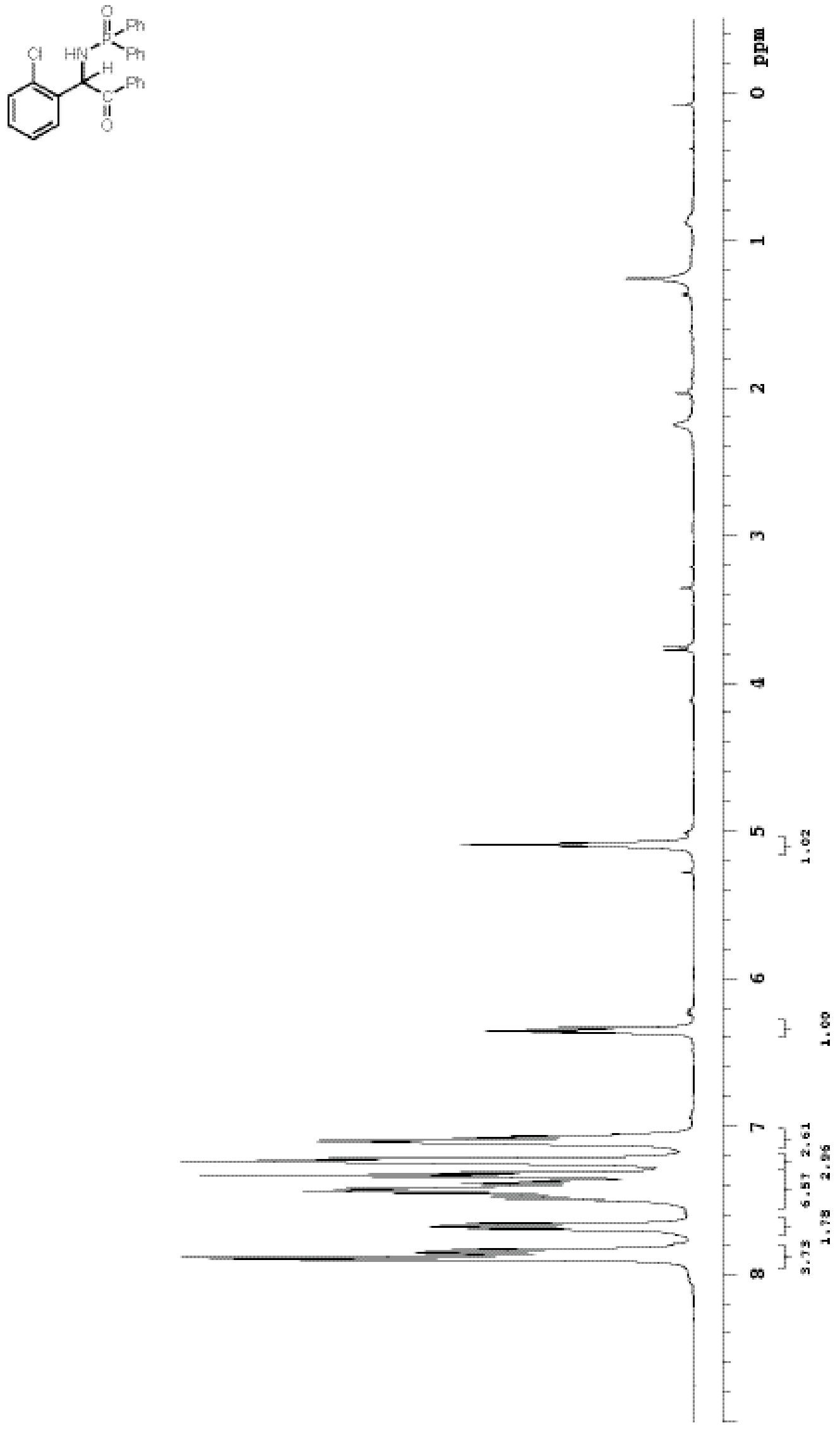

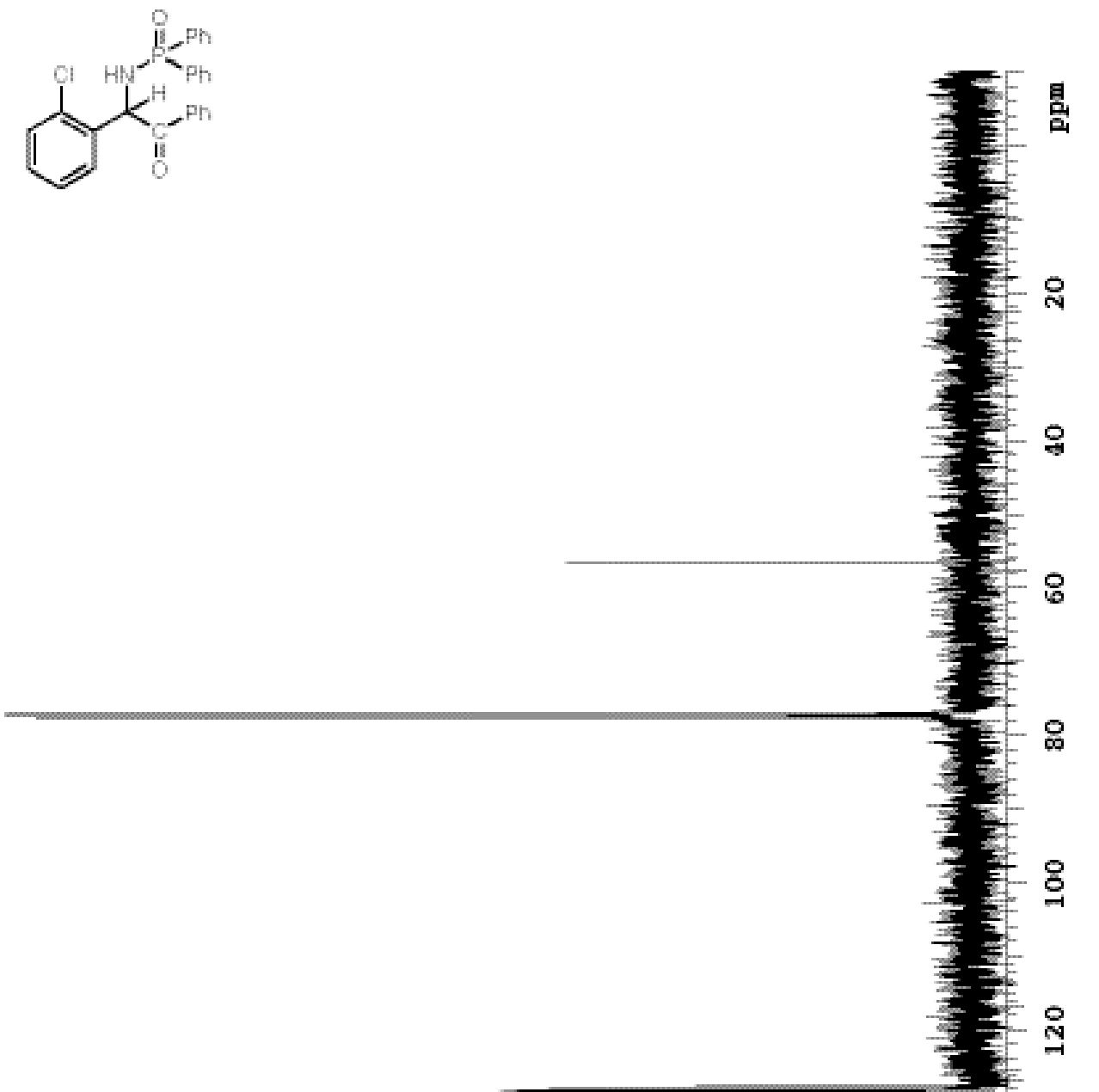

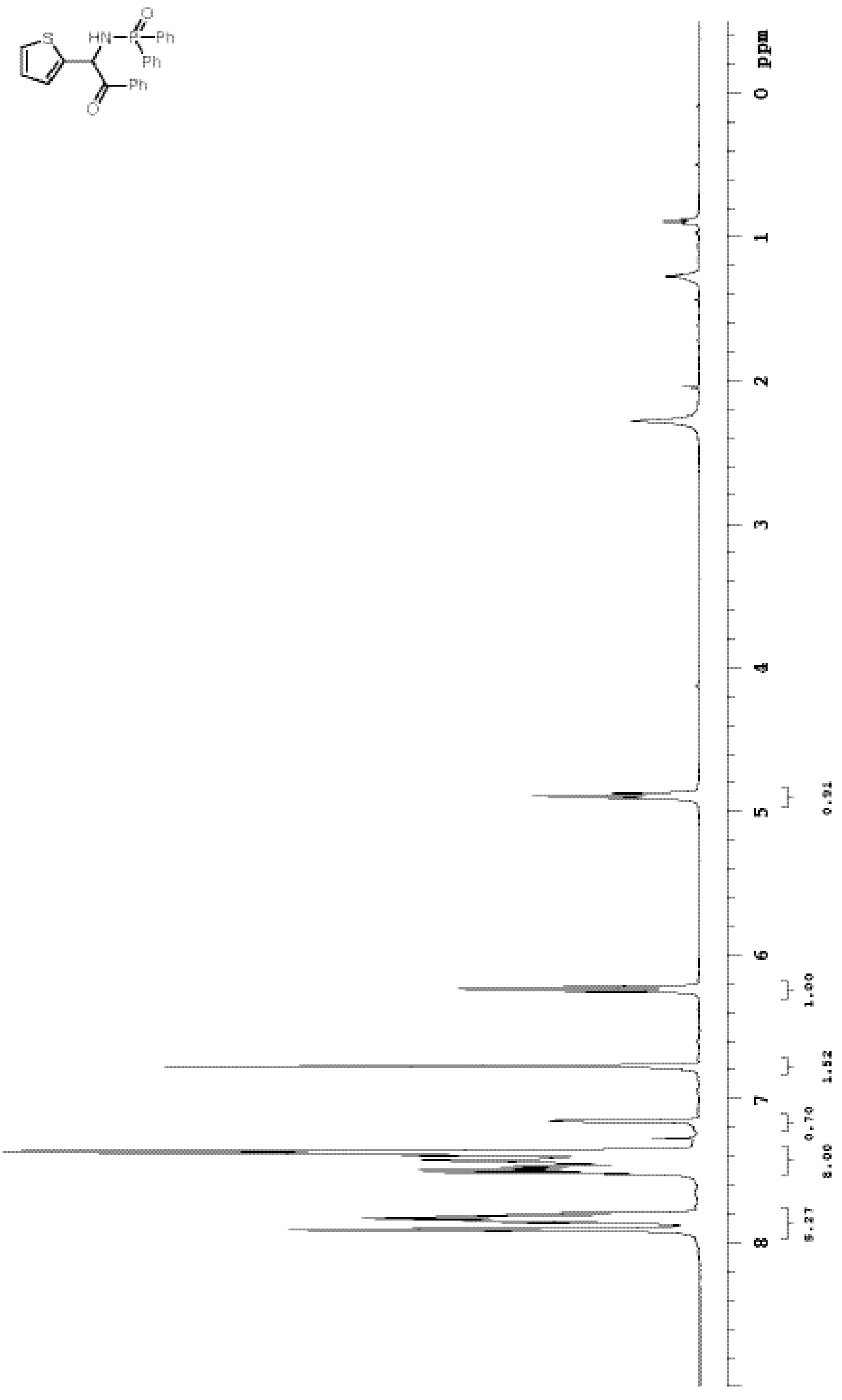


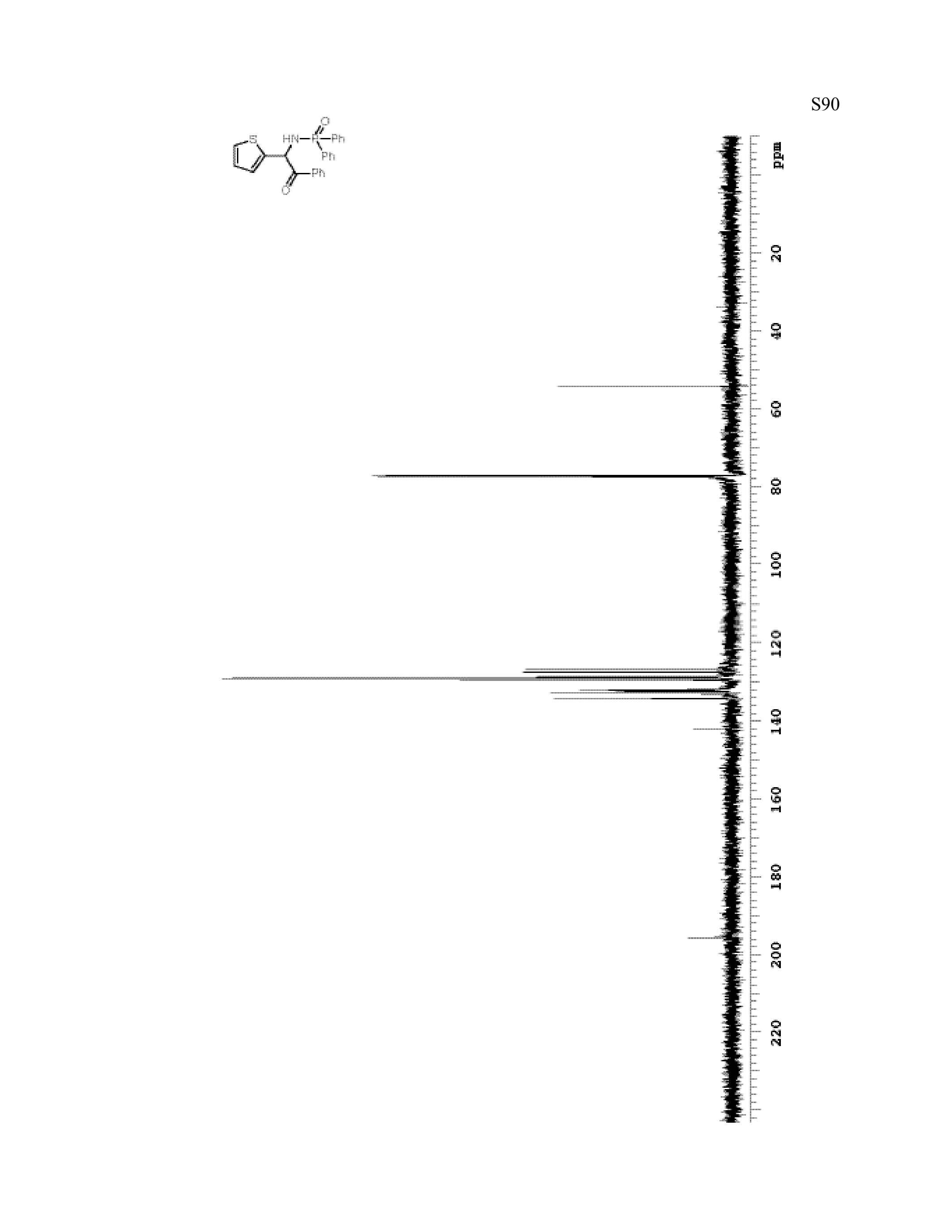

